Commun. Math. Phys. 128, 333-391 (1990)

Communications in

Mathematical

Physics

(C) Springer-Verlag 1990

\title{
Mean-Field Critical Behaviour for Percolation in High Dimensions
}

\author{
Takashi Hara ${ }^{1, \star}$ and Gordon Slade ${ }^{2, \star \star}$ \\ ${ }^{1}$ Courant Institute of Mathematical Sciences, N.Y.U., 251 Mercer Street, New York, NY 10012, \\ USA \\ ${ }^{2}$ Department of Mathematics and Statistics, McMaster University, Hamilton, Ontario, Canada \\ L8S 4K 1
}

\begin{abstract}
The triangle condition for percolation states that $\sum_{x, y} \tau(0, x) \tau(x, y)$ $\cdot \tau(y, 0)$ is finite at the critical point, where $\tau(x, y)$ is the probability that the sites $x$ and $y$ are connected. We use an expansion related to the lace expansion for a self-avoiding walk to prove that the triangle condition is satisfied in two situations: (i) for nearest-neighbour independent bond percolation on the $d$-dimensional hypercubic lattice, if $d$ is sufficiently large, and (ii) in more than six dimensions for a class of "spread-out" models of independent bond percolation which are believed to be in the same universality class as the nearest-neighbour model. The class of models in (ii) includes the case where the bond occupation probability is constant for bonds of length less than some large number, and is zero otherwise. In the course of the proof an infrared bound is obtained. The triangle condition is known to imply that various critical exponents take their mean-field (Bethe lattice) values $\left(\gamma=\beta=1, \delta=\Delta_{t}=2, t \geqq 2\right)$ and that the percolation density is continuous at the critical point. We also prove that $v_{2}=1 / 2$ in (i) and (ii), where $v_{2}$ is the critical exponent for the correlation length.
\end{abstract}

\section{Table of Contents}

1. Introduction . . . . . . . . . . . . . . . . . . . . . . 334

1.1. The Models. . . . . . . . . . . . . . . . . . . . . . . . 335

1.2. Main Results . . . . . . . . . . . . . . . . . . . . . . . . . 337

1.3. Overview of the Proof . . . . . . . . . . . . . . . . 339

1.4. Organization, Notation, and Definitions. . . . . . . . . . . . 346

2. The Expansion. . . . . . . . . . . . . . . . . . . . . . . . 348

2.1. Derivation of the Expansion . . . . . . . . . . . . . . . 349

* Supported by the Nishina Memorial Foundation and NSF grant PHY-8896163. Address after September 1, 1989: Department of Mathematics, University of Texas at Austin, Austin, TX 78712, USA. Electronic address: hara @ emx.utexas.edu

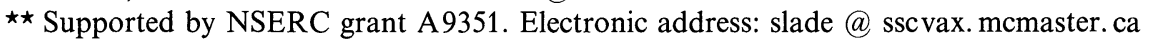


2.2. Bounds in $x$-Space for Each Term of the Expansion . . . . . . . . . . . . . 354

2.3. Bounds in $k$-Space for Each Term of the Expansion . . . . . . . . . . . . . 358

3. Diagrammatic Estimates . . . . . . . . . . . . . . . . . 360

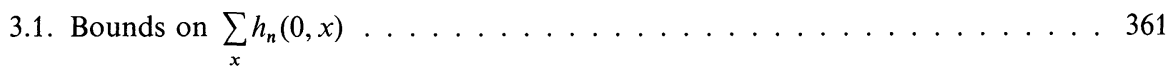

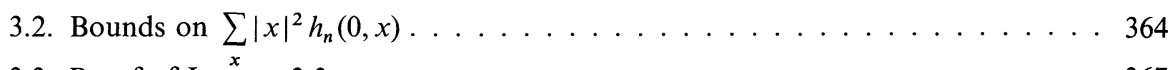

3.3. Proof of Lemma $3.3 \ldots \ldots$. . . . . . . . . . . . . . . . . 367

4. Proof of the Main Results (Theorem 1.1) for the Nearest-Neighbour Model. . . . . . 368

4.1. General Structure . . . . . . . . . . . . . . . . . . . . 368

4.2. Proof of Lemmas 4.1 and $4.2 \ldots \ldots \ldots$. . . . . . . . . . . . . . . . . . . . . . . . . 370

4.3. Proof of Proposition 4.3 . . . . . . . . . . . . . . . . 371

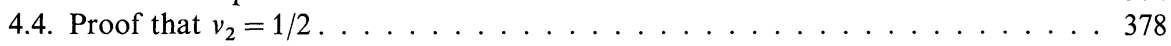

5. Proof of the Main Results (Theorem 1.2) for the Spread-Out Models . . . . . . . . . 379

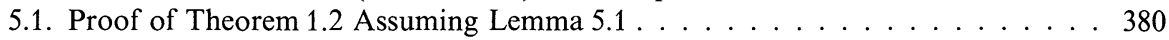

5.2. Proof of Lemma $5.1 \ldots \ldots$. . . . . . . . . . . . . . . . 383

\section{Introduction}

In the last decade significant progress has been made in the rigorous understanding of critical phenomena, particularly concerning its mean-field behaviour in high dimensions. For Ising and $\varphi^{4}$ spin systems one of the important ideas has been the combination of the infrared bound [16] with correlation inequalities. This method, together with other ideas, has led to considerable understanding of these models in four or more dimensions, including a proof of the triviality of $\varphi^{4}$ field theories in more than four dimensions and a proof that many critical exponents take their mean-field values above four dimensions $[35,1,15,4,3]$.

For the self-avoiding walk no general proof of an infrared bound is known, and the methods which were successful for the spin systems cannot be applied. An alternate approach was introduced by Brydges and Spencer [12], who used the lace expansion to prove mean-field critical behaviour for the weakly self-avoiding walk in more than four dimensions. This method was further developed in [31-33], where mean-field critical behaviour was proved for the strictly self-avoiding walk above some undetermined dimension $d_{0}>4$, and in [25], where the infinite selfavoiding walk was constructed in high dimensions.

For percolation there is also no general proof of an infrared bound, and in fact there are indications that the infrared bound is violated in less than six dimensions $[14,41]$. However based on an analogy with the bubble diagram, which played an important role in the analysis of the Ising and $\varphi^{4}$ models, Aizenman and Newman [6] introduced an unverified condition, the so-called triangle condition, which was shown by them to imply mean-field behaviour for the susceptibility in percolation models. The triangle condition is expected to hold above six dimensions. Subsequently further implications of the triangle condition were obtained in $[8,28]$. In this paper we prove that the triangle condition is satisfied in two situations: (i) for independent nearest-neighbour Bernoulli bond percolation in sufficiently high dimensions, and (ii) in more than six dimensions for a class of "spread-out" models of independent bond percolation, which includes certain 
finite range models as well as certain models in which the bond occupation probability decays exponentially as a function of the length of the bond. In the course of the proof we obtain a uniform infrared bound below the critical point, which is valid in high dimensions for model (i), and above six dimensions for the models in (ii). The method of proof can also be applied to site percolation, and yields the same results (with a suitable interpretation of the spread-out models). Consequences of the triangle condition are that the critical exponents $\gamma, \beta, \delta$, and $\Delta_{t}$ (defined below) exist and take their mean-field values, and that the percolation density is continuous at the critical point.

The models in class (ii) are believed to be in the same universality class as the nearest neighbour-model. It is known that for the nearest neighbour model the upper critical dimension is at least six [13,38], and the proof of this fact can be extended to the models in (ii) [39]. Thus our result strongly supports the conjecture that for these models the upper critical dimension is equal to six.

The proof of these results is based on an expansion for the two-point function which is related to the lace expansion for self-avoiding walk. The expansion is used to treat a percolation model as a perturbation of the random walk model whose transition probabilities are proportional to the percolation bond occupation probabilities. Similar methods can also be applied to branched polymers [21].

\subsection{The Models}

We consider independent Bernoulli bond percolation on the infinite $d$ dimensional hypercubic lattice $\mathbb{Z}^{d}$. To each unordered bond (pair of distinct sites) $b=\{x, y\}\left(x, y \in \mathbb{Z}^{d}\right)$ a random variable $n_{b}$ is associated, which takes the values 0 and 1 . The set of random variables $\left\{n_{b}\right\}$ is independent, and the distribution of $n_{b}$ is given by

$$
\operatorname{Prob}\left(n_{b}=1\right)=p_{b}, \quad \operatorname{Prob}\left(n_{b}=0\right)=1-p_{b} .
$$

We require $\mathbb{Z}^{d}$-invariance (translation, reflection and rotation by $\pi / 2$ ) for the $p_{\{x, y\}}=p_{\{0, y-x\}}$.

We consider the following possibilities for $p_{b}$ :

(i) the nearest-neighbour model:

$$
p_{\{0, x\}}= \begin{cases}p & \text { if } x \text { is a nearest neighbour of } 0 \\ 0 & \text { otherwise. }\end{cases}
$$

(ii) The spread-out models:

$$
p_{\{0, x\}}=p \cdot L^{-d} g(x / L),
$$

where $g: \mathbb{R}^{d} \rightarrow[0, \infty)$ is a given function which is normalized so that $\int g(x) d^{d} x=1$, and is invariant under rotations by $\pi / 2$ and reflections in the coordinate hyperplanes. The parameter $L$ will be taken to be large. (This type of limit to study mean-field behaviour is related to the so-called Kac limit $[23,26]$.) A basic example is

$$
g(x)= \begin{cases}1 & \text { if }\|x\|_{\infty} \equiv \max _{1 \leqq i \leqq d}\left|x_{i}\right| \leqq 1 \\ 0 & \text { otherwise }\end{cases}
$$


We require that $g$ decay exponentially at infinity (i.e., there exist $C, \varepsilon>0$ such that $\left.g(x) \leqq C \exp \left[-\varepsilon\|x\|_{\infty}\right]\right)$. Then models (i) and (ii) are expected to be in the same universality class. The bond density $p$ is the only parameter in these models (apart from an additional parameter $h$ we shall mention briefly to define the critical exponent $\delta$ ). For the models in (ii) we will show that the triangle condition is satisfied for $d>6$, if $L$ is sufficiently large and $g$ is piecewise differentiable.

If $n_{b}=1$ we say that $b$ is occupied, while if $n_{b}=0$ we say that $b$ is vacant. We use $\operatorname{Prob}_{p}(E)$ to denote the probability of an event $E$ with respect to the joint distribution of the $\left\{n_{b}\right\}$, and denote expectation with respect to this distribution by $\langle\cdot\rangle_{p}$.

Given a bond configuration $\left\{n_{b}\right\}$, two sites $x$ and $y$ in the lattice are said to be connected if there exists a path from $x$ to $y$ which consists of occupied bonds. The connected cluster $C(x)$ of $x$ is the random set of sites defined by

$$
C(x)=\left\{y \in \mathbb{Z}^{d}: y \text { is connected to } x\right\} .
$$

The number of sites in $C(x)$ is denoted by $|C(x)|$.

We define the two-point function

$$
\tau_{p}(x, y)=\operatorname{Prob}_{p}(y \text { is connected to } x),
$$

the susceptibility

$$
\chi(p)=\sum_{x} \tau_{p}(0, x)=\langle|C(0)|\rangle_{p},
$$

the percolation density

$$
P_{\infty}(p)=\operatorname{Prob}_{p}(|C(0)|=\infty),
$$

and the magnetization

$$
M(p, h)=1-\sum_{1 \leqq n<\infty} e^{-h n} \operatorname{Prob}_{p}(|C(0)|=n) .
$$

We also define two correlation lengths

$$
\xi(p)=-\left[\lim _{n \rightarrow \infty} \frac{1}{n} \ln \tau_{p}(0,(n, 0, \ldots, 0))\right]^{-1}
$$

and

$$
\xi_{2}(p)=\left(\frac{\sum_{x}|x|^{2} \tau_{p}(0, x)}{\sum_{x} \tau_{p}(0, x)}\right)^{1 / 2} .
$$

To simplify the notation we will often omit the subscript $p$.

For the nearest-neighbour model it has been known for thirty years that (except for the trivial case $d=1$ ) there is a critical value $p_{c} \in(0,1)$ (depending on the dimension) such that the percolation density vanishes for $p<p_{c}$ and is nonzero for $p>p_{c}[10,19]$. Such a critical value of $p$ also exists for the models in (ii), for the $g$ 's we will consider. Recently it has been proved that $p_{c}$ can also be characterized as $\sup \{p: \chi(p)<\infty\}[27,2]$. In this paper we are concerned with the critical 
behaviour of the model, i.e., the behaviour of functions such as those defined above in the vicinity of $(p, h)=\left(p_{c}, 0\right)$. By analogy with other statistical mechanical models, and in agreement with numerical calculations, this behaviour is expected to be in the form of power laws, and we introduce the critical exponents $\gamma, \beta, \delta, \Delta_{t+1}, \nu$ and $\nu_{2}$ as follows:

$$
\begin{gathered}
\chi(p) \sim\left(p_{c}-p\right)^{-\gamma} \text { as } p \uparrow p_{c}, \\
P_{\infty}(p) \sim\left(p-p_{c}\right)^{\beta} \text { as } p \downarrow p_{c}, \\
M\left(p_{c}, h\right) \sim h^{1 / \delta} \text { as } h \downarrow 0, \\
\left\langle|C(0)|^{t+1}\right\rangle \mid\left\langle|C(0)|^{t}\right\rangle \sim\left(p_{c}-p\right)^{-\Delta_{t+1}} \text { as } p \uparrow p_{c}, \\
\xi(p) \sim\left(p_{c}-p\right)^{-v} \text { as } p \uparrow p_{c}, \\
\xi_{2}(p) \sim\left(p_{c}-p\right)^{-v_{2}} \text { as } p \uparrow p_{c} .
\end{gathered}
$$

Here $f(p) \sim\left|p-p_{c}\right|^{-\lambda}$ is defined to mean that there exist positive constants $C_{1}$ and $C_{2}$ such that

$$
C_{1}\left|p-p_{c}\right|^{-\lambda} \leqq f(p) \leqq C_{2}\left|p-p_{c}\right|^{-\lambda} .
$$

There are other critical exponents that can be defined (see [17, 24, 37]), but these are the ones for which we can conclude mean-field values. The exponent $\delta$ is also often defined by the (formally) equivalent relation

$$
\operatorname{Prob}_{p_{c}}(|C(0)|=n) \sim n^{-1-1 / \delta} .
$$

So far very little has been rigorously proved about the existence of the above critical exponents. See [17,24] for a review.

On the Bethe lattice (Cayley tree), it can be shown that $\gamma, \beta, \delta$, and $\Delta_{t+1}$ exist and have the values $\gamma=\beta=1, \delta=\Delta_{t+1}=2$ for $t+1=2,3,4, \ldots$ [17]. The Bethe lattice critical exponents are known as the mean-field values, and it is expected that for the models (i) and (ii) in more than six dimensions all critical exponents take their mean-field values. The definition of $v$ and $v_{2}$ is problematic on the Bethe lattice since these exponents are defined using the Euclidean structure, but with a proper definition it can be shown that $v_{2}=1 / 2$ [17]. On the hypercubic lattice it is expected that $v$ and $v_{2}$ are equal, so the mean field values of these exponents is $1 / 2$. On the other hand, it has been rigorously shown by Chayes and Chayes [13] and by Tasaki [38] that if all the critical exponents exist, then they cannot simultaneously take their mean-field values in any dimension less than six. Thus the upper critical dimension of the system is expected to be six.

\subsection{Main Results}

Aizenman and Newman [6] introduced an unverified condition, the triangle condition, and showed that it implies that (1.7) holds with $\gamma=1$. The triangle condition states that

$$
\nabla\left(p_{c}\right)<\infty,
$$


where $\nabla(p)$ is the triangle diagram

$$
\nabla(p)=\sum_{x, y} \tau_{p}(0, x) \tau_{p}(x, y) \tau_{p}(y, 0)
$$

Since the susceptibility $\chi(p)=\sum_{x} \tau(0, x)$ diverges as $p$ approaches $p_{c}$ from below for the models (i) and (ii) we are considering [6], the statement that the triangle diagram is finite at the critical point is not without content. Denoting by $\hat{\tau}_{p}(k)$ the Fourier transform of the two-point function, defined by

$$
\hat{\tau}_{p}(k)=\sum_{x} \tau_{p}(0, x) e^{i k \cdot x},
$$

the triangle diagram can be written

$$
\nabla(p)=(2 \pi)^{-d} \int_{[-\pi, \pi]^{d}} d^{d} k \hat{\tau}_{p}(k)^{3} .
$$

If an infrared bound

$$
\hat{\tau}_{p}(k) \leqq C(p) k^{-2}, \text { as } k \rightarrow 0,
$$

were known, it would then follow that $\nabla(p)<\infty$ for $d>6$. A uniform bound on $C(p)$ for $p<p_{c}$ would thus be tantamount to (1.13). Such a uniform bound, and hence the triangle condition, is expected to hold in more than six dimensions.

In addition to the fact that the triangle condition implies $\gamma=1$, Barsky and Aizenman [8] have shown that the triangle condition implies that (1.8) and (1.9) hold with $\beta=1$ and $\delta=2$, and Nguyen [28] has shown that it implies that (1.10) holds with $\Delta_{t}=2$ for $t=2,3,4, \ldots$. It follows from (1.8) that the percolation density $P_{\infty}$ is continuous at the critical point, a fact which has been proved until now only for $d=2$ [30]. (Continuity of $P_{\infty}$ has been shown for all other values of $p$ in [5].) Our main result is that the triangle condition is satisfied (i) for the nearestneighbour model if the dimension $d$ is sufficiently large, and (ii) for the spread-out models if $L$ is sufficiently large, for $d>6$. Hence all the above consequences of this condition also hold in these situations.

In this paper we prove the following theorems.

Theorem 1.1. For nearest-neighbour independent Bernoulli bond percolation on $\mathbb{Z}^{d}$ there is a $d_{0}>6$ such that for $d \geqq d_{0}$ the infrared bound

$$
\hat{\tau}_{p}(k) \leqq \text { const } k^{-2} \text {, uniformly in } p<p_{c}
$$

holds. In addition the triangle condition is satisfied, i.e.,

$$
\nabla\left(p_{c}\right)=\sum_{x, y} \tau_{p_{c}}(0, x) \tau_{p_{c}}(x, y) \tau_{p_{c}}(y, 0)<\infty .
$$

Theorem 1.2. The infrared bound

$$
\hat{\tau}_{p}(k) \leqq \text { const } k^{-2} \text {, uniformly in } p<p_{c},
$$

and the triangle condition both hold for $d>6$, for the spread-out models (ii), if $L$ is sufficiently large (depending on $d$ and $g$ ) and if $\frac{\partial^{d} g}{\partial x_{1} \ldots \partial x_{d}}$ is piecewise continuous 
and $g$ satisfies the following conditions:

$$
\begin{gathered}
g e^{\delta\|x\|_{1}} \in L_{\infty}\left(\mathbb{R}^{d}\right) \text { for some } \delta>0, \\
\int g(x) d^{d} x=1,
\end{gathered}
$$

$\int\left|\partial^{I} g(x)\right| d^{d} x<\infty$, where the derivative is interpreted as a distribution, and

$$
\partial^{I}=\prod_{\mu \in I} \frac{\partial}{\partial x_{\mu}} \text { and } I \subset\{1,2, \ldots, d\},
$$

$g$ is invariant under rotations by $\pi / 2$ and reflections in the coordinate hyperplanes.

As a consequence of the proofs of these theorems, we will also show that the exponent $v_{2}$ for the correlation length $\xi_{2}$ exists and takes its mean-field value

$$
v_{2}=1 / 2
$$

for the nearest neighbour model if $d \geqq d_{0}$ and for the spread-out models if $d>6$ and $L \geqq L_{0}$. However a more complicated analysis is required to control the correlation length $\xi$. It is proved in [20] that $v=1 / 2$, under the same hypotheses as (1.15).

Also, Eq. (4.10) below gives an upper bound for the critical probability for the nearest-neighbour model in high dimensions, which together with a well-known lower bound states that there is a constant $C>0$ (independent of $d$ ) such that

$$
\frac{1}{2 d-1} \leqq p_{c} \leqq \frac{1}{2 d}+\frac{C}{d^{2}} \text {. }
$$

We have done little to obtain the best possible value of $d_{0}$ in Theorem 1.1. Our current best estimate is $d_{0}=48$, obtained by a slightly more complicated analysis than that presented in this paper. This value can doubtless be improved, but a new idea will be needed to obtain the triangle condition for the nearest-neighbour model right down to the expected upper critical dimension of six. The fact that we are unable to do much better than $d_{0}=48$ suggests that we still do not have a very efficient expansion for percolation. (For the self-avoiding walk the situation is better: the lace expansion can be used to show that the bubble diagram is finite and $\gamma=1, v_{2}=1 / 2$ for the self-avoiding walk if $d \geqq 7$ [22]. Here $\gamma$ is the exponent which measures the rate of divergence of the generating function at the critical point, and $v_{2}$ is defined as for percolation. The upper critical dimension for self-avoiding walk is expected to be 4 , so $d \geqq 5$ should be optimal.) However in view of the fact that all of the models we are considering are believed to be in the same universality class, the conjecture of universality, together with Theorem 1.2, strongly supports mean-field behaviour for the nearest-neighbour model in more than six dimensions.

The method of proof involves an expansion whose convergence is assured by taking $1 / d$ to be small in Theorem 1.1 and $1 / L$ to be small in Theorem 1.2.

\subsection{Overview of the Proof}

In this section we describe the general structure of the proof of Theorem 1.1. The same ideas are used to prove Theorem 1.2. The basic structure of the proof is the 
same as that used in [31], where in particular it was shown that the bubble diagram for the self-avoiding walk is finite at the critical point, in sufficiently high dimensions. (Proofs with this type of structure, in different contexts, can be found in $[11,36]$.) In order to focus on the main ideas here we make some simplifications and omit some details, deferring the complete proof to the remainder of the paper.

We define the following quantities:

$$
\begin{gathered}
T(p)=\nabla(p)-1=\sum_{x, y} \tau(0, x) \tau(x, y) \tau(y, 0)-\tau(0,0)^{3}, \\
W(p)=\sum_{x}|x|^{2} \tau(0, x)^{2} .
\end{gathered}
$$

The massless gaussian propagator is given by

$$
C(x, y)=(2 \pi)^{-d} \int d^{d} k \frac{e^{i k \cdot(y-x)}}{1-D(k)}, \text { where } D(k)=d^{-1} \sum_{\mu=1}^{d} \cos k_{\mu} .
$$

We also introduce the gaussian quantities $T_{G}$ and $W_{G}$ corresponding to $T(p)$ and $W(p)$, defined by replacing $\tau$ by $C$ in (1.16) and (1.17). It is not difficult to show that for $d \geqq 7$ there are constants $K_{1}$ and $K_{2}$ such that $T_{G} \leqq K_{1} d^{-1}$ and $W_{G} \leqq K_{2} d^{-1}$ (e.g., using Appendix B of [20] or Lemma 3.1 of [31]).

1.3.1. General Framework. The proof that the triangle condition is satisfied in high dimensions is accomplished by showing that the following three statements hold:

(i) For $p<p_{c}, T(p)$ and $W(p)$ are continuous functions of $p$.

(ii) For $p \leqq 1 / 2 d, T(p) \leqq T_{G} \leqq K_{1} d^{-1}$ and $W(p) \leqq W_{G} \leqq K_{2} d^{-1}$.

(iii) Let $d$ be sufficiently large and fix any $p \in\left[1 / 2 d, p_{c}\right)$. If

$$
T(p) \leqq 4 K_{1} d^{-1}, W(p) \leqq 4 K_{2} d^{-1} \text {, and } 2 d p \leqq 4 \text {, }
$$

then in fact

$$
T(p) \leqq 3 K_{1} d^{-1}, W(p) \leqq 3 K_{2} d^{-1} \text {, and } 2 d p \leqq 3 .
$$

(In reality the precise statement of (iii) is more involved and can be found in Sect. 4.) In the course of the proof of (iii) the infrared bound is obtained.

Together (i), (ii) and (iii) imply that there is a forbidden region in the graph of $T(p)$ or $W(p)$, as depicted in Fig. 1. Therefore (1.19) holds and hence by definition of $T(p)$ it follows that

$$
\nabla(p) \leqq 1+3 K_{1} d^{-1}, \text { for all } p<p_{c} .
$$

But since $\tau_{p}(x, y)$ is an increasing and continuous function of $p$ [5], it follows from (1.20) and the monotone convergence theorem that

$$
\nabla\left(p_{c}\right)=\lim _{p \uparrow p_{c}} \nabla(p) \leqq 1+3 K_{1} d^{-1}
$$

and hence the triangle condition holds.

The proofs of (i) and (ii) are simple and are given in Sect. 4.2. The difficult part of the proof is to obtain (iii). This is done by obtaining an expression for the Fourier transform $\hat{\tau}_{p}(k)$, which under the assumption (1.18) can be shown to be a 


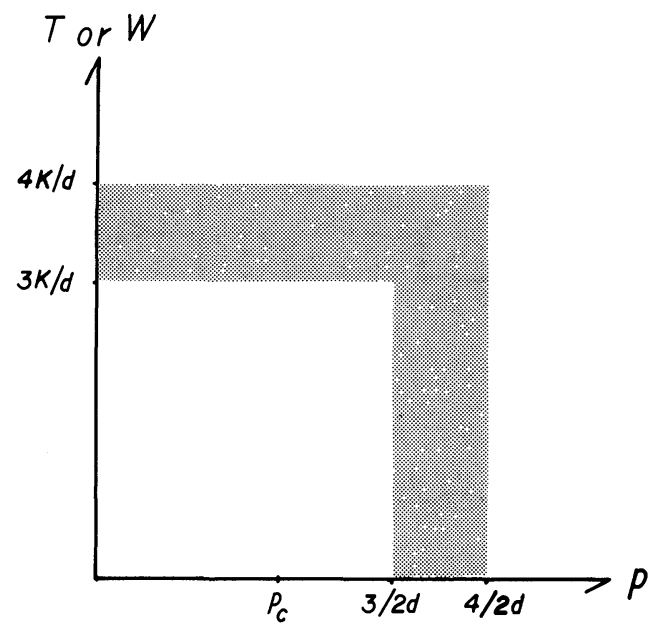

Fig. 1. Forbidden region (shaded) in the graph of $T$ or $W$ versus $p$, for $p<p_{c}$

small perturbation of the massless gaussian propagator $\hat{C}(k)=[1-D(k)]^{-1}$. Hence $T(p)$ and $W(p)$ are close to their gaussian counterparts $T_{G}$ and $W_{G}$, and thus the improved bounds on $T(p)$ and $W(p)$ in (1.19) follow.

For the self-avoiding walk the analogue of step (iii) was obtained using the lace expansion. Here we derive and use an analogous expansion for percolation, which yields an expression for $\hat{\tau}_{p}(k)$. The lace expansion was derived in [12] using an expansion followed by resummation, but it can also be derived using the inclusionexclusion relation [34]. It is the latter approach that we use for percolation. (In a similar spirit, Park [29] has used the inclusion-exclusion relation to study intersection probabilities for simple random walk.)

1.3.2. The Expansion. We describe the expansion in detail in Sect. 2, but let us here give the basic idea of the expansion and explain how it can be used to obtain (iii). We begin with some definitions. Two sites $x$ and $y$ are said to be doubly connected (in a given configuration) if there exist two self-avoiding walks from $x$ to $y$, consisting of occupied bonds, which are distinct in the sense that they do not share a common bond (although they may share common sites). If $x$ and $y$ are connected, but not doubly connected, then there must be at least one bond in the connected cluster of $x$ whose removal would disconnect $x$ and $y$. Such a bond is referred to as a pivotal bond for the connection of $x$ and $y$. There is a natural order for the set of pivotal bonds, namely the first pivotal bond is the pivotal bond $b=\{u, v\}$ such that one endpoint, say $u$, of $b$ is doubly connected to $x$. Either the other endpoint $v$ of $b$ is doubly connected to $y$, in which case there are no further pivotal bonds for the connection of $x$ and $y$, or it is not. In the latter case, the second pivotal bond is the one for which one endpoint is doubly connected to $v$, and so on. This leads to the picture of a cluster joining $x$ and $y$ shown in Fig. 2. This picture was also used in [27]. It is convenient to always regard a site as being doubly connected to itself. In Fig. 2(b), the pivotal bonds divide the cluster into parts which are mutually avoiding in the sense that no two can share a common 
(a)

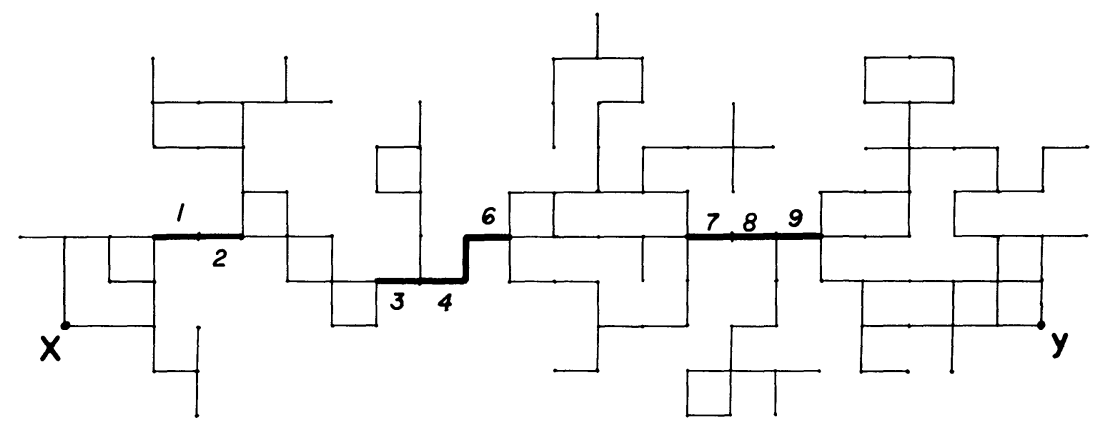

(b)

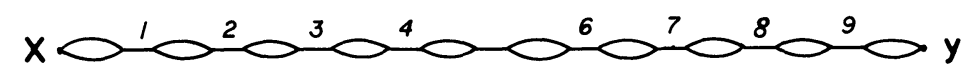

Fig. 2. (a) A configuration in which $x$ and $y$ are connected. (b) Schematic representation of the configuration in (a)

site. This represents a kind of repulsive interaction similar to that of self-avoiding walk.

The event that 0 is connected to $x$ is then the disjoint union of the event that 0 is doubly connected to $x$ and the event that there is a pivotal bond for the connection (and hence a first pivotal bond for the connection). We denote the former event symbolically as

$\{0$ is doubly connected to $x\}=0 \longleftrightarrow x$,

and the latter as

$$
\{0 \text { is connected to } x \text { but not doubly }\}=\bigcup_{(u, v)} 0 \succ_{u} x .
$$

Here the ordered bond $(u, v)$ is the first pivotal bond for the connection, so 0 is doubly connected to $u$. The union in (1.21) is disjoint. Using this notation, and the convention that $\tau(x, x)=1$, we have

$$
\tau(0, x)=\operatorname{Prob}(0 \longleftrightarrow x)+\sum_{(u, v)} \operatorname{Prob}\left(0 \sum_{u} \cdot-x\right) .
$$

At this point we need to make the following definitions.

Definition 1.3. Given a bond configuration $\left\{n_{b}\right\}$ and a set $A$ of sites, we define

(a) the connected cluster of $x$ in $\mathbb{Z}^{d} \backslash A$ :

$\mathrm{C}^{A}(x)=\left\{y \in \mathbb{Z}^{d}: y\right.$ and $x$ are connected using bonds having no endpoint in $\left.A\right\}$. If $y \in C^{A}(x)$ we say that $y$ and $x$ are connected in $\mathbb{Z}^{d} \backslash A$,

(b) the reduced two-point function:

$$
\tau_{p}^{A}(x, y)=\operatorname{Prob}_{p}\left(y \in C^{A}(x)\right)
$$

(c) the connected cluster of $x$ after the bond $\{u, v\}$ has been made vacant:

$\widetilde{C}^{\{u, v\}}(x)=\left\{y \in C(x): y\right.$ remains connected to $x$ after $n_{\{u, v\}}$ is set equal to zero $\}$. 
Now we use a lemma from [6] (which is stated and proved in Sect. 2.1) to write the summand in the second term on the right side of Eq. (1.22) as follows:

$$
\text { Prob }\left(0 \bigotimes_{u} \quad x\right)=p\left\langle I[0 \circlearrowright u] \tau^{\tilde{C}^{(u, v)}(0)}(v, x)\right\rangle \text {. }
$$

The factor of $p$ on the right side is due to the fact that $\{u, v\}$ is occupied, while the restricted two-point function and the double connection of 0 and $u$ are due to the fact that $(u, v)$ is the first pivotal bond. Next we replace the restricted two-point function in (1.23) by

$$
\tau^{\widetilde{C}^{(u, v)}(0)}(v, x)=\tau(v, x)-\left[\tau(v, x)-\tau^{\tilde{C}^{(u, v)}(0)}(v, x)\right] .
$$

This yields the equation

$$
\begin{aligned}
\tau(0, x)= & \operatorname{Prob}(0 \circlearrowright x)+p \sum_{(u, v)} \operatorname{Prob}(0 \circlearrowright u) \tau(v, x) \\
& -p \sum_{(u, v)}\left\langle I[0 \circlearrowright u]\left(\tau(v, x)-\tau^{\tilde{C}^{(u, v\}}(0)}(v, x)\right)\right\rangle .
\end{aligned}
$$

Our goal is to manipulate the right-hand side of (1.25) so as to obtain terms which either involve a convolution with $\tau$ evaluated at $x$, as in the second term on the right side, or involve a multiple connection from $x$ and no explicit two-point function, as in the first term on the right side. (The reason for this will become clear when we take Fourier transforms.) To this end we first observe that $\tau(v, x)-\tau^{\tilde{C}^{(u, v)}(0)}(v, x)=\left\langle I\left[v\right.\right.$ is connected to $x$ in $\mathbb{Z}^{d}$ but not in $\left.\left.\mathbb{Z}^{d} \backslash \widetilde{C}^{\{u, v\}}(0)\right]\right\rangle$.

The event on the right side of (1.26) is such that every occupied self-avoiding walk from $v$ to $x$ must pass through $\widetilde{C}^{\{u, v\}}(0)$ (which within the inner expectation (1.26) on the right side of (1.25) represents a given deterministic fixed set of sites). As an example of a situation that can occur in this event, we consider the configuration in Fig. 3. There $\left(u_{1}, v_{1}\right)$ is the first pivotal bond such that every occupied self-avoiding walk from $v$ to $u_{1}$ passes through $\widetilde{C}^{\{u, v\}}(0)$, and $z^{\prime}$ is the

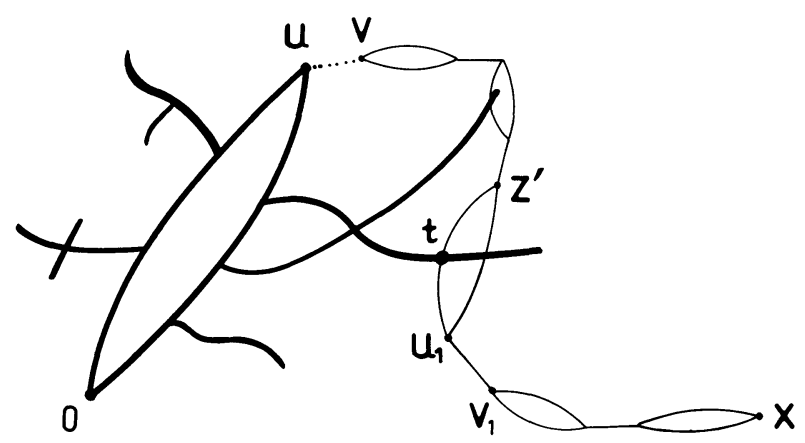

Fig. 3. An example of a configuration contributing to the right side of Eq. (1.26). Thick lines represent the bonds connecting the sites in $\widetilde{C}^{\{u, v\}}(0)$. Thin lines represent the bonds connecting $v$ and $x$. The point $t$ is a point in $\widetilde{C}^{\{u, v\}}(0)$ on an occupied self-avoiding walk from $z^{\prime}$ to $u_{1}$ 
latter endpoint of the previous pivotal bond $\left\{z, z^{\prime}\right\}$. Other possibilities and special cases exist, for example $v$ could be doubly connected to $x$, but we ignore such cases in this overview. By another application of the lemma quoted above (1.23), the contribution to (1.26) due to the configuration in Fig. 3 is equal to

$$
\sum_{\left(u_{1}, v_{1}\right)} \sum_{z^{\prime}}\left\langle I\left[E\left(v, z^{\prime},\left(u_{1}, v_{1}\right)\right)\right] \tau^{\tilde{C}^{\left(u_{1}, v_{1}\right)}(v)}\left(v_{1}, x\right)\right\rangle,
$$

where $E\left(v, z^{\prime},\left(u_{1}, v_{1}\right)\right)$ is the event that (a) $v$ is connected to $z^{\prime}$ via a pivotal bond $\left\{z, z^{\prime}\right\}$, (b) $z^{\prime}$ is doubly connected to $u_{1}$, with one of the occupied paths passing through a site in $\widetilde{C}^{\{u, v\}}(0)$, and (c) $\left\{u_{1}, v_{1}\right\}$ is occupied and is the first pivotal bond such that every occupied self-avoiding walk from $v$ to $u_{1}$ passes through $\widetilde{C}^{\{u, v\}}(0)$. As before we will replace the restricted two-point function in (1.27) using the analogue of (1.24).

This allows us to write (1.25) as

$$
\begin{aligned}
\tau(0, x)= & \operatorname{Prob}(0 \longleftrightarrow x)+p \sum_{(u, v)} \operatorname{Prob}(0 \circlearrowright u) \tau(v, x) \\
& -p \sum_{\substack{(u, v) \\
\left(u_{1}, v_{1}\right)}} \sum_{z^{\prime}}\left\langle I[0 \circlearrowright u]\left\langle I\left[E\left(v, z^{\prime},\left(u_{1}, v_{1}\right)\right)\right]\right\rangle\right\rangle \tau\left(v_{1}, x\right) \\
& + \text { other cases + remainder. }
\end{aligned}
$$

The remainder comes from the use of (1.24) in (1.27). Let us now for simplicity ignore the other cases and the remainder in (1.28). We take the Fourier transform of (1.28), and use the fact that the Fourier transform of a convolution is the product of the Fourier transforms. Solving the resulting equation for $\hat{\tau}_{p}(k)$ yields

$$
\hat{\tau}(k)=\frac{1+\hat{g}_{0}(k)}{1-2 d p D(k)-\hat{\Pi}_{0}(k)+\hat{\Pi}_{1}(k)}+\text { corrections, }
$$

where

$$
\begin{gathered}
\hat{g}_{0}(k)=\sum_{x \neq 0} \operatorname{Prob}(0 \circlearrowright x) e^{i k \cdot x}, \\
2 d p D(k)+\hat{\Pi}_{0}(k)=p \sum_{(u, v)} \operatorname{Prob}(0 \circlearrowright u) e^{i k \cdot v},
\end{gathered}
$$

(the first term on the left side of (1.31) is the $u=0$ term on the right side) and

$$
\hat{\Pi}_{1}(k)=p \sum_{\substack{(u, v) \\\left(u_{1}, v_{1}\right)}} \sum_{z^{\prime}}\left\langle I[0 \circlearrowright u]\left\langle I\left[E\left(v, z^{\prime},\left(u_{1}, v_{1}\right)\right)\right]\right\rangle\right\rangle e^{i k \cdot v_{1}} .
$$

1.3.3. Bounding Terms in the Expansion. We can estimate $\hat{g}_{0}, \hat{\Pi}_{0}$, and $\hat{\Pi}_{1}$ using the van den Berg-Kesten inequality [9]. In the form that we need it the van den Berg-Kesten inequality states the following. Let $V_{1}, \ldots, V_{n}$ be sets of paths in the lattice, and let $E_{i}, i=1, \ldots, n$, be the event that at least one of the paths in $V_{i}$ is occupied. Then

Prob [there exist pairwise distinct occupied paths $\omega_{1} \in V_{1}, \ldots, \omega_{n} \in V_{n}$ ]

$$
\leqq \operatorname{Prob}\left[E_{1}\right] \ldots \operatorname{Prob}\left[E_{n}\right] \text {. }
$$


The inequality (1.33) with $n=2$ can be used to estimate (1.30) and (1.31) by taking $V_{1}=V_{2}$ to be the set of all paths from 0 to $x$ :

$$
\begin{gathered}
\left|\hat{g}_{0}(k)\right| \leqq \sum_{x \neq 0} \tau(0, x)^{2} \leqq T(p) \\
\left|\hat{\Pi}_{0}(k)\right| \leqq 2 d p \sum_{u \neq 0} \operatorname{Prob}(0 \longleftrightarrow u) \leqq 2 d p \sum_{x \neq 0} \tau(0, x)^{2} \leqq 2 d p T(p) .
\end{gathered}
$$

The estimation of (1.32) is more involved. We will use the inequality

$$
I\left[E\left(v, z^{\prime},\left(u_{1}, v_{1}\right)\right] \leqq \sum_{t} I\left[t \in \tilde{C}^{\{u, v\}}(0)\right] I\left[\bar{E}\left(v, z^{\prime}, t,\left(u_{1}, v_{1}\right)\right)\right]\right.
$$

where $\bar{E}\left(v, z^{\prime}, t,\left(u_{1}, v_{1}\right)\right)$ is the event that (a) $v$ is connected to $z^{\prime}$, (b) $z^{\prime}$ is doubly connected to $u_{1}$, with one of the occupied paths passing through $t$, (c) $\left(u_{1}, v_{1}\right)$ is occupied, and (d) all of these connections are given by distinct paths. With (1.33), this gives

$$
\begin{aligned}
\left|\hat{\Pi}_{1}(k)\right| & \leqq p \sum_{\substack{(u, v) \\
\left(u_{1}, v_{1}\right)}} \sum_{t, z^{\prime}}\left\langle I[0 \longleftrightarrow u] I\left[t \in \widetilde{C}^{\{u, v\}}(0)\right]\left\langle I\left[\bar{E}\left(v, z^{\prime}, t,\left(u_{1}, v_{1}\right)\right)\right]\right\rangle\right\rangle \\
& \leqq 2 d p^{2} \sum_{(u, v), u_{1}, t, z^{\prime}}\left\langle I[0 \longleftrightarrow u] I\left[t \in \tilde{C}^{\{u, v\}}(0)\right]\right\rangle \tau\left(v, z^{\prime}\right) \tau\left(z^{\prime}, t\right) \tau\left(t, u_{1}\right) \tau\left(z^{\prime}, u_{1}\right) \\
& \leqq 2 d p^{2} \sum_{(u, v), u_{1}, t, z^{\prime}, w} \tau(0, u) \tau(0, w) \tau(w, u) \tau(w, t) \tau\left(v, z^{\prime}\right) \tau\left(z^{\prime}, t\right) \tau\left(t, u_{1}\right) \tau\left(z^{\prime}, u_{1}\right) .
\end{aligned}
$$

To better visualize this inequality we will introduce a diagrammatic notation in which it is represented by

$$
\left|\hat{\Pi}_{1}\right| \leqq 2 d p \text { कू }
$$

(One factor of $p$ is inherent in the diagram.) As we shall show in Sects. 2.3 and 3.1, the right side of (1.37) can be bounded in terms of $T(p)$ to give

$$
\left|\hat{\Pi}_{1}\right| \leqq(2 d p)^{2}(1+T(p))^{2}\left[T(p) / 3+(2 T(p) / 3 d)^{1 / 2}+1 / d\right] .
$$

The right side of (1.38) is $O\left(d^{-1}\right)$ for large $d$, under the assumption (1.18). Analogous upper bounds can be obtained for first and second $k$-derivatives of $\hat{g}_{0}$, $\hat{\Pi}_{0}$, and $\hat{\Pi}_{1}$ involving $W(p)$ as well as $T(p)$.

1.3.4. Bounds on $\hat{\tau}(k)$. Proof Completed. In this section we describe how (1.19) can be obtained, assuming (1.18). Incorporating the corrections in Eq. (1.29) into corrections to $\hat{g}_{0}$ and $\hat{\Pi}_{0}-\hat{\Pi}_{1}, \hat{\tau}(k)$ can be written in the form

$$
\hat{\tau}(k)=\frac{1+\hat{g}(k)}{1-2 d p D(k)-\hat{\Pi}(k, p)},
$$

where

$$
\hat{g}(k)=\hat{g}_{0}(k)+\text { corrections }
$$

and

$$
\hat{\Pi}(k, p)=\hat{\Pi}_{0}(k)-\hat{\Pi}_{1}(k)+\text { corrections } .
$$


Now for any $p<p_{c}$,

$$
\begin{aligned}
1-2 d p D(k)-\hat{\Pi}(k, p)= & 1-2 d p D(k)-\hat{\Pi}(k, p)-[1-2 d p-\hat{\Pi}(0, p)] \\
& +[1-2 d p-\hat{\Pi}(0, p)] .
\end{aligned}
$$

Using (1.18), it follows from (1.34) that $1+\hat{g}(0) \approx 1$ for large $d$, and hence (since $\hat{\tau}(0)=\chi(p))$ the last term on the right side is

$$
1-2 d p-\hat{\Pi}(0, p)=[1+\hat{g}(0)] \chi(p)^{-1} \approx \chi(p)^{-1} \geqq 0 .
$$

Therefore

$$
\hat{\tau}(k) \leqq \frac{1+|\hat{g}(k)|}{2 d p(1-D(k))+\hat{\Pi}(0, p)-\hat{\Pi}(k, p)} .
$$

The difference of $\hat{\Pi}$ 's in the denominator can be controlled in terms of the second $k$-derivative of $\hat{\Pi}$ using (1.18), along the lines we have outlined above for bounding $\hat{\Pi}_{1}$. The assumption on $W$ in (1.18) is used at this point. This allows us to absorb the difference of $\hat{\Pi}$ 's into the gaussian term $2 d p(1-D(k))$, which with (1.34) yields an infrared bound. The fact that $2 d p>1$ is used at this point. We then write $T(p)$ and $W(p)$ in terms of $\hat{\tau}(k)$, and using this infrared bound obtain the improved estimate (1.19) for $T(p)$ and $W(p)$. The inequality $2 d p \leqq 3$ follows from (1.40) and the fact that $\hat{\Pi}$ is $O\left(d^{-1}\right)$. Although the proof of the infrared bound initially uses the assumption (1.18), once we have derived the stronger statement (1.19) the assumption holds automatically.

This completes the outline of the proof. The remainder of the paper is devoted to giving a rigorous proof of Theorems 1.1 and 1.2, following this outline. In the next section we describe the organization of the remainder of the paper and summarize some notation and definitions.

\subsection{Organization, Notation and Definitions}

In this section we describe the organization of the remainder of the paper and summarize some definitions. We also introduce a convenient diagrammatic notation.

In Sect. 2 a detailed derivation of the expansion for the two-point function is given, and estimates are given for the terms of the expansion in both $x$-space and $k$ space. These estimates are given by diagrams as in (1.37). In Sect. 3 it is shown how the diagrams can be bounded by products of $T, W$ and a small number of related quantities which play a role in the precise version of the assumption (1.18). In Sect. 4 the statements (i), (ii) and the precise version of (iii) (from Sect. 1.3.1) are proved, completing the proof of Theorem 1.1. In Sect. 4.4 the proof that $v_{2}=1 / 2$ for the nearest-neighbour model when $d \geqq d_{0}$ is given. Finally in Sect. 5 the modifications to the proof of Theorem 1.1 which are necessary to prove Theorem 1.2 are described.

We now list several definitions, some of which were made in the last section and some of which are new.

Definition 1.4. (a) A bond is an unordered pair of distinct sites $\{x, y\}$. An ordered bond is denoted $(x, y)$. A path from $x$ to $y$ is a self-avoiding walk (not necessarily 
nearest-neighbour for the spread-out models) from $x$ to $y$, considered as a set of bonds. Given a bond configuration $\left\{n_{b}\right\}$, an occupied path is a path consisting of occupied bonds. Two paths are distinct if they have no bonds in common. (Distinct paths may have common sites.)

(b) Given a bond configuration, two sites $x$ and $y$ are connected if there is an occupied path from $x$ to $y$. They are doubly connected if there are at least two distinct occupied paths from $x$ to $y$. A site $x$ is always considered to be doublyconnected to itself.

(c) Given a set of sites $A \subset \mathbb{Z}^{d}$ and a bond configuration, two sites $x$ and $y$ are connected in $A$ if there is an occupied path from $x$ to $y$ consisting of bonds whose endpoints both lie in $A$. The sites $x$ and $y$ are doubly-connected in $A$ if there are at least two distinct occupied paths from $x$ to $y$ consisting of bonds whose endpoints both lie in $A$. Two sites $x$ and $y$ are connected through $A$ if there is at least one occupied path from $x$ to $y$ and if in addition every occupied path from $x$ to $y$ has at least one bond with an endpoint in $A$. Similarly we define $x$ and $y$ to be doublyconnected through $A$ if $x$ and $y$ are doubly-connected and connected through $A$.

(d) Given a bond configuration, the connected cluster $C(x)$ of $x$ is given by

$$
C(x)=\left\{y \in \mathbb{Z}^{d}: y \text { is connected to } x\right\} .
$$

Given a set $A$ of sites, we define

$$
C^{A}(x)=\left\{y \in \mathbb{Z}^{d}: y \text { and } x \text { are connected in } \mathbb{Z}^{d} \backslash A\right\},
$$

and the restricted two-point function

$$
\tau_{p}^{A}(x, y)=\operatorname{Prob}_{p}\left(y \in C^{A}(x)\right) .
$$

Given a bond $\{u, v\}$, we define

$$
\begin{gathered}
\tilde{C}^{\{u, v\}}(x)=\left\{y \in \mathbb{Z}^{d}: y \text { is connected to } x\right. \text { in the new configuration } \\
\text { obtained by setting } \left.n_{\{u, v\}}=0\right\} .
\end{gathered}
$$

(e) Given a bond configuration, a bond $\{u, v\}$ (occupied or not) is called pivotal for the connection from $x$ to $y$ if either $x \in C(u)$ and $y \in C(v)$, or $x \in C(v)$ and $y \in C(u)$, but $y \notin \widetilde{C}^{\{u, v\}}(x)$. Similarly an ordered bond $(u, v)$ is pivotal for the connection from $x$ to $y$ if $x \in \widetilde{C}^{\{u, v\}}(u), y \in \widetilde{C}^{\{u, v\}}(v), y \notin \widetilde{C}^{\{u, v\}}(x)$. If $x$ and $y$ are connected then there is a natural order to the set of occupied pivotal bonds for the connection from $x$ to $y$ (providing there exists one or more occupied pivotal bond), and each of these pivotal bonds can be ordered in a natural way, as follows. The first pivotal bond from $x$ to $y$ is the ordered occupied pivotal bond $(u, v)$ such that $u$ is doubly connected to $x$. If $(u, v)$ is the first pivotal bond for the connection from $x$ to $y$, then the second pivotal bond is the first pivotal bond for the connection from $v$ to $y$, and so on.

Finally we describe the diagrammatic notation that will be used for the remainder of the paper. This notation has the virtue of making cumbersome expressions more transparent. We will denote the event that there is an occupied path between $x$ and $y$ by a solid line joining $x$ and $y: x-y$. In a diagram 
consisting of several such solid lines it is always to be understood that there are distinct paths making the required connections. For example, the diagram

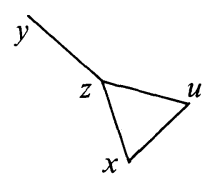

denotes the event that $y$ is connected to $z, z$ is connected to $u, z$ is connected to $x$, and $x$ is connected to $u$, all by distinct paths.

We will also use a Feynman diagram notation in which full propagators are represented by wavy lines, unlabelled vertices are summed over the lattice, and labelled vertices are fixed. In addition we will shade in any loop for which all vertices on the loop may coincide. In unshaded loops the summation is constrained such that at least two of the vertices must be distinct. For example the diagram

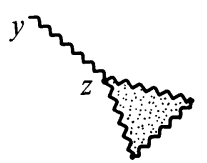

represents

$$
\tau(y, z) \sum_{u, x} \tau(z, u) \tau(u, x) \tau(x, z)=\tau(y, z) \nabla(p),
$$

while the diagram

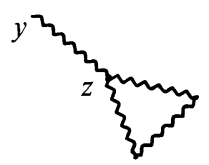

represents

$$
\tau(y, z)\left[\sum_{u, x} \tau(z, u) \tau(u, x) \tau(x, z)-\tau(z, z)^{3}\right]=\tau(y, z)[\nabla(p)-1] .
$$

A pair of wavy lines terminating together in bars denotes two propagators ending at two sites $y$ and $y^{\prime}$ and carries a factor of $p_{\left\{y, y^{\prime}\right\}}$ and a sum over all ordered bonds $\left(y, y^{\prime}\right)$. For example,

$$
0 \sum_{\text {minn }}^{\frac{3}{5}}=\sum_{\left(y, y^{\prime}\right)} p_{\left\{y, y^{\prime}\right\}} \tau(0, y) \tau\left(y^{\prime}, 0\right) .
$$

\section{The Expansion}

In this section we derive the expansion for $\tau_{p}(0, x)$ which is the main tool used in the proof of Theorems 1.1 and 1.2. The results of this section are valid for general $\mathbb{Z}^{d}$-invariant bond percolation models, long or short range. (In fact, Propositions 2.3 and 2.4 hold without the assumption of $\mathbb{Z}^{d}$-invariance.) To simplify the 
notation we write $p_{x y}$ for $p_{\{x, y\}}$. In Sect. 2.1 we use the inclusion-exclusion principle to prove an identity of the form:

$$
\begin{aligned}
\tau(0, x)=\delta_{0, x} & +\sum_{n=0}^{N}(-1)^{n} g_{n}(0, \mathrm{x})+(-1)^{N+1} R_{N}(0, x)+\sum_{y \neq 0} p_{0 y} \tau(y, x) \\
& +\sum_{n=0}^{N}(-1)^{n} \sum_{y^{\prime}} \Pi_{n}\left(0, y^{\prime}\right) \tau\left(y^{\prime}, x\right) .
\end{aligned}
$$

The identity (2.1) is valid for any $p<p_{c}$ and any nonnegative integer $N$, and the nonnegative quantities $g_{n}, \Pi_{n}$ and $R_{N}$ are given by explicit formulas in Proposition 2.3. There is a close relation between $\Pi_{n}$ and $g_{n}$ :

$$
\Pi_{n}\left(0, y^{\prime}\right)=\sum_{y} g_{n}(0, y) p_{y y^{\prime}}
$$

In Sect. 2.2 the van den Berg-Kesten inequality is used to obtain $x$-space estimates for $g_{n}, \Pi_{n}$ and $R_{N}$ in terms of the full propagator $\tau$ itself. These estimates are stated in Proposition 2.4 .

Taking the Fourier transform of Eq. (2.1) gives the following formula for $\hat{\tau}(k)$, which will play a key role in obtaining the infrared bound in Sect. 4:

$$
\hat{\tau}(k)=\frac{1+\sum_{n=0}^{N}(-1)^{n} \hat{g}_{n}(k)+(-1)^{N+1} \hat{R}_{N}(k)}{1-\sum_{y \neq 0} p_{0 y} e^{i k \cdot y}-\sum_{n=0}^{N}(-1)^{n} \hat{\Pi}_{n}(k)} .
$$

To control $\hat{\tau}(k)$ we will employ $k$-space estimates for $\hat{g}_{n}(k), \hat{\Pi}_{n}(k)$ and $\hat{R}_{N}(k)$. These follow easily from the $x$-space estimates and are given in Proposition 2.6 in Sect. 2.3.

Throughout this section we make use of the definitions and notation given in Sect. 1.4.

\subsection{Derivation of the Expansion}

The expansion is derived using a lemma from [6] together with repeated use of inclusion-exclusion. We begin (as outlined in Sect. 1.3.2) by writing

$\tau(0, x)=\operatorname{Prob}(0$ is connected to $x)$

$$
\begin{aligned}
= & \operatorname{Prob}(0 \longleftrightarrow x)+\sum_{(u, v)} \operatorname{Prob}(0 \text { is connected to } x \text { but not doubly, } \\
& \text { and }(u, v) \text { is the first pivotal bond }) .
\end{aligned}
$$

We define

$$
g_{0}(0, x)=\operatorname{Prob}(0 \longleftrightarrow x)-\delta_{0, x} .
$$

To analyze the second term on the right side of (2.3), we use the lemma from [6].

Before stating the lemma, we first introduce some definitions. Let $B$ be a random or deterministic set of bonds, and let $E$ be any event. We denote by $B_{s}$ the set of sites consisting of endpoints of bonds in $B$, and we denote by $\widetilde{C}^{\{u, v\}}(0)_{b}$ the 
connected bond cluster of the origin which remains after setting $n_{\{u, v\}}=0$. The event that $E$ occurs on $B$ is defined to be the set consisting of those bond configurations for which the new configuration obtained by setting $n_{b}=0$ for all $b \notin B$ is a configuration in the event $E$. The lemma involves events satisfying:

$E$ occurs and the ordered bond $(u, v)$ is pivotal for the connection from 0 to $x$

$$
\Rightarrow E \text { occurs on } \tilde{C}^{\{u, v\}}(0)_{b} \text {. }
$$

An example of an $E$ satisfying (2.5) is the event that 0 is doubly connected to $u$.

For simplicity we consider only the case $p<p_{c}$, which is sufficient for our needs.

Lemma 2.1. Let $E$ be an event satisfying (2.5), and fix $p<p_{c}$. Then

$\langle I[E$ occurs and $(u, v)$ is occupied and pivotal for the connection from 0 to $x]\rangle$ $=p_{u v}\left\langle I[E\right.$ occurs and $\left.u \in C(0)] \tau^{\tau^{(u, v)}(0)}(v, x)\right\rangle$.

Proof. The proof is by conditioning on $\tilde{C}^{\{u, v\}}(0)$. Since $p<p_{c}$, this cluster is finite with probability one. Let

$\mathscr{E}=\left\{\right.$ finite connected bond clusters $B: 0, u \in B_{s}$ and $E$ occurs on $\left.B\right\}$.

Since $E$ satisfies (2.5), apart from a set of measure zero

$\{E$ occurs and $(u, v)$ is pivotal $\}=\bigcup_{B \in \mathscr{E}}\left\{\tilde{C}^{\{u, v\}}(0)_{b}=B\right.$ and $(u, v)$ is pivotal $\}$.

Therefore

$\langle I[E$ occurs and $(u, v)$ is occupied and pivotal $]\rangle$

$=p_{u v}\langle I[E$ occurs and $(u, v)$ is pivotal $]\rangle$

$=p_{u v} \sum_{B \in \mathscr{E}} \operatorname{Prob}\left((u, v)\right.$ is pivotal $\left.\mid \widetilde{C}^{\{u, v\}}(0)_{b}=B\right) \operatorname{Prob}\left(\widetilde{C}^{\{u, v\}}(0)_{b}=B\right)$.

Since

$$
\begin{aligned}
& \left\{\tilde{C}^{\{u, v\}}(0)_{b}=B \text { and }(u, v) \text { is pivotal }\right\} \\
= & \left\{\tilde{C}^{\{u, v\}}(0)_{b}=B \text { and } v \text { is connected to } x \text { in } \mathbb{Z}^{d} \backslash B_{s}\right\},
\end{aligned}
$$

and since the events $\left\{\tilde{C}^{\{u, v\}}(0)_{b}=B\right\}$ and $\left\{v\right.$ is connected to $x$ in $\left.\mathbb{Z}^{d} \backslash B_{s}\right\}$ are independent, the conditional probability is equal to

$$
\left\langle I\left[v \text { is connected to } x \text { in } \mathbb{Z}^{d} \backslash B_{s}\right]\right\rangle=\tau^{B_{s}}(v, x) .
$$

Therefore the above sum can be written

$$
\begin{aligned}
& p_{u v} \sum_{B \in \mathscr{E}} \tau^{B_{s}}(v, x)\left\langle I\left[\tilde{C}^{\{u, v\}}(0)_{b}=B\right]\right\rangle=p_{u v} \sum_{B \in \mathscr{E}}\left\langle\tau^{\left\{\tilde{C}^{(u, v\}}(0)\right.}(v, x) I\left[\widetilde{C}^{\{u, v\}}(0)_{b}=B\right]\right\rangle \\
& =p_{u v}\left\langle I\left[u \in \tilde{C}^{\{u, v\}}(0) \text { and } E \text { occurs on } \tilde{C}^{\{u, v\}}(0)\right] \tau^{\tilde{C}^{\{u, v\}}(0)}(v, x)\right\rangle \\
& =p_{u v}\left\langle I\left[u \in \widetilde{C}^{\{u, v\}}(0) \text { and } E \text { occurs }\right] \tau^{\tilde{C}^{\{u, v\}}(0)}(v, x)\right\rangle \text {. }
\end{aligned}
$$

The last equality is due to the fact that if $E$ occurs, but not on $\tilde{C}^{\{u, v\}}(0)_{b}$, then by $(2.5)(u, v)$ cannot be pivotal, in which case either $v \in \widetilde{C}^{\{u, v\}}(0)$ or $v$ is not connected to $x$, and hence $\tau^{\tilde{C}^{(u, v)}(0)}(v, x)=0$. 

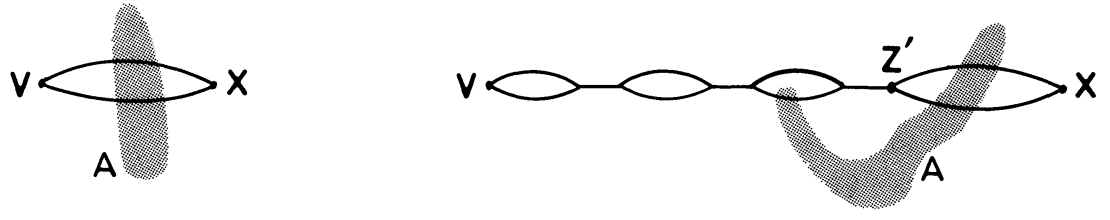

Fig. 4. Examples of configurations contributing to $I_{D}(v, x ; A)$

But now if $u \in C(0) \backslash \widetilde{C}^{\{u, v\}}(0)$ then 0 is connected to $u$ through $\{v\}$, and hence $\tau^{\widetilde{C}^{\{u, v\}}(0)}(v, x)=0$. Thus the right side of $(2.6)$ is equal to

$$
p_{u v}\left\langle I[E \text { occurs and } u \in C(0)] \tau^{\widetilde{C}^{\{u, v\}}(0)}(v, x)\right\rangle \text {, }
$$

and the lemma is proved.

We now use this lemma, with $E$ the event that 0 is doubly connected to $u$, to write the second term on the right side of (2.3) as

$$
\sum_{(u, v)} p_{u v}\left\langle I[0 \longleftrightarrow u] \tau^{\tilde{C}^{\{u, v\}}(0)}(v, x)\right\rangle .
$$

To implement the inclusion-exclusion principle, we replace the restricted two point function by

$$
\tau^{\tilde{C}^{\{u, v\}}(0)}(v, x)=\tau(v, x)-\left[\tau(v, x)-\tau^{\tilde{C}^{\{u, v\}}(0)}(v, x)\right] .
$$

This gives (2.1) with $N=0$, if we define

and

$$
\Pi_{0}\left(0, y^{\prime}\right)=\sum_{y \neq 0} p_{y y^{\prime}}\langle I[0 \longleftrightarrow y]\rangle=\sum_{y} p_{y y^{\prime}} g_{0}(0, y)
$$

$$
R_{0}(0, x)=\sum_{(u, v)} p_{u v}\left\langle I[0 \longleftrightarrow u]\left\{\tau(v, x)-\tau^{\tilde{C}^{(u, v\}}(0)}(v, x)\right\}\right\rangle .
$$

To proceed further we rewrite the difference of two-point functions occurring in the remainder $R_{0}$ using the following lemma. In preparation for the statement of the lemma we make the following definition, which is illustrated in Fig. 4. Given a set $A$ of sites, define

$$
\begin{aligned}
& I_{D}(v, x ; A)=I[v \text { and } x \text { are doubly connected through } A] \\
& +I\left[\exists z^{\prime} \neq v \text { such that } z^{\prime} \text { is connected to } v \text { in } \mathbb{Z}^{d} \backslash A,\right.
\end{aligned}
$$
$z^{\prime}$ is an endpoint of a pivotal bond for the connection of $v$ and $x$, and $z^{\prime}$ is doubly connected to $x$ through $A$ ].

Lemma 2.2. Given a set $A$ of sites and two fixed sites $v$ and $x$,

$$
\tau(v, x)-\tau^{A}(v, x)=\left\langle I_{D}(v, x ; A)\right\rangle+\sum_{\left(y, y^{\prime}\right)} p_{y y^{\prime}}\left\langle I_{D}(v, y ; A) \tau^{\tilde{C}^{\left\{y, y^{\prime}\right\}}(v)}\left(y^{\prime}, x\right)\right\rangle .
$$

Proof. By definition of $\tau^{A}$,

$$
\tau(v, x)-\tau^{A}(v, x)=\langle I[v \text { and } x \text { are connected through } A]\rangle .
$$


Each configuration contributing to the right side belongs to exactly one of the following two cases.

Case 1. The sites $v$ and $x$ are doubly connected through $A$. This contributes to $I_{D}$, giving the first term on the right side of (2.9).

Case 2. The sites $v$ and $x$ are connected through $A$ but are not doubly connected through $A$. In this case there is at least one pivotal bond for the connection from $v$ to $x$, and we subdivide this case into the following cases.

Case $2 a$. There is no pivotal bond $\left(w, w^{\prime}\right)$ (for the connection from $v$ to $x$ ) such that $w$ is connected to $v$ through $A$. This gives the other contribution to $I_{D}$ in (2.9). There $z^{\prime}$ is the latter endpoint of the last pivotal bond for the connection of $v$ to $x$. Case $2 b$. There is a pivotal bond whose first endpoint is connected to $v$ through $A$. In this case there is a first such pivotal bond $\left(y, y^{\prime}\right)$. The contribution due to this case to $\tau(v, x)-\tau^{A}(v, x)$ is

\section{$\sum_{\left(y, y^{\prime}\right)}\left\langle I\left[\left(y, y^{\prime}\right)\right.\right.$ is the first pivotal bond (for the connection from $v$ to $x$ )
whose first endpoint is connected to $v$ through $A]\rangle$.}

Let $E$ be the event that $v$ and $y$ are connected through $A$, and there is no pivotal bond $\left(w, w^{\prime}\right)$ for the connection from $v$ to $y$ such that $w$ is connected to $v$ through $A$. Then $E$ satisfies $(2.5)$ (with $(u, v)$ replaced by $\left(y, y^{\prime}\right)$ and 0 by $v$ ) and $I[E]=I_{D}(v, y ; A)$, and hence by Lemma 2.1 the above expression is equal to the last term on the right side of the equation in the statement of the lemma. This completes the proof of Lemma 2.2.

We now return to the derivation of the expansion. Beginning with the equation

$$
\tau(0, x)=\delta_{x, 0}+g_{0}(0, x)+\sum_{v \neq 0} p_{0 v} \tau(v, x)+\sum_{y^{\prime}} \Pi_{0}\left(0, y^{\prime}\right) \tau\left(y^{\prime}, x\right)-R_{0}(0, x),
$$

in which $g_{0}, \Pi_{0}$ and $R_{0}$ were defined in Eqs. (2.4), (2.8a), and (2.8b), we use Lemma 2.2, with $A=\tilde{C}^{\{u, v\}}(0)$, to replace the difference of two-point functions occurring in $R_{0}$. This leads to a nested expectation in $R_{0}$. In order to specify unambiguously to which expectation the set $\widetilde{C}^{\{u, v\}}(0)$ (which is defined in terms of a given bond configuration) corresponds, we use subscripts in nested expectations, i.e., $\widetilde{C}_{n}^{\{u, v\}}(0)$ denotes $\widetilde{C}^{\{u, v\}}(0)$ defined by the bond configuration corresponding to the configuration $\langle\cdot\rangle_{(n)}$. In applying Lemma 2.2 to $(2.8 \mathrm{~b}), \widetilde{C}^{\{u, v\}}(0)$ is random in the expectation of $(2.8 \mathrm{~b})$, but deterministic with respect to the expectation produced by application of Lemma 2.2. Using subscripts we have

$$
\begin{aligned}
R_{0}(0, x)= & \sum_{(u, v)} p_{u v}\left\langle I[0 \circlearrowright u]\left\langle I_{D}\left(v, x ; \tilde{C}_{0}^{\{u, v\}}(0)\right)\right\rangle_{(1)}\right\rangle_{(0)} \\
& +\sum_{(u, v)} p_{u v}\left\langle I[0 \circlearrowright u] \sum_{\left(y, y^{\prime}\right)} p_{y y^{\prime}}\left\langle I_{D}\left(v, y ; \tilde{C}_{0}^{\{u, v\}}(0)\right) \tau^{\tilde{C}_{1}^{\left(j, y^{\prime \prime}\right.}(v)}\left(y^{\prime}, x\right)\right\rangle_{(1)}\right\rangle_{(0)} .
\end{aligned}
$$

The first term gives $g_{1}$. In the second term we replace the restricted two-point function $\tau_{\tilde{C}_{1}^{(j, y)}(v)}$ by an unrestricted two-point function plus a correction as in (2.7). The term involving the unrestricted two-point function gives rise to a term $\sum_{y^{\prime}} \Pi_{1}\left(0, y^{\prime}\right) \tau\left(y^{\prime}, x\right)$, where $\Pi_{1}$ (like $\left.g_{1}\right)$ contains two nested expectation values. 
The correction term, containing a difference of restricted and unrestricted twopoint functions, is the remainder $R_{1}(0, x)$. Now the same procedure can be iterated, beginning with the use of Lemma 2.2 to rewrite the difference of twopoint functions, and so on. The details are straightforward but tedious, and lead to the following result. To abbreviate the notation, we write

$$
\tilde{C}_{j-1}=\tilde{C}_{j-1}^{\left\{y_{j} y_{j}^{\prime}\right\}}\left(y_{j-1}^{\prime}\right) \text {, }
$$

and

$$
I_{D}^{j}=I_{D}\left(y_{j}^{\prime}, y_{j+1} ; \widetilde{C}_{j-1}\right) .
$$

Proposition 2.3. For $N \geqq 0$ and for $p<p_{c}$,

$$
\begin{aligned}
\tau(0, x)=\delta_{x, 0} & +\sum_{n=0}^{N}(-1)^{n} g_{n}(0, x)+\sum_{v \neq 0} p_{0 v} \tau(v, x) \\
& +\sum_{n=0}^{N}(-1)^{n} \sum_{y^{\prime}} \Pi_{n}\left(0, y^{\prime}\right) \tau\left(y^{\prime}, x\right)+(-1)^{N+1} R_{N}(0, x),
\end{aligned}
$$

where

$$
g_{0}(0, x)=\operatorname{Prob}(0<x)-\delta_{0, x},
$$

and for $n \geqq 1$,

$$
\begin{aligned}
g_{n}(0, x)= & \sum_{\left(y_{1}, y_{1}^{\prime}\right)} p_{y_{1} y_{1}^{\prime}} \cdots \sum_{\left(y_{n}, y_{n}^{\prime}\right)} p_{y_{n}, y_{n}^{\prime}}\left\langleI [ 0 \circlearrowright y _ { 1 } ] \left\langleI _ { D } ^ { 1 } \left\langleI _ { D } ^ { 2 } \left\langleI _ { D } ^ { 3 } \cdots \left\langle I_{D}^{n-1}\right.\right.\right.\right.\right. \\
& \left.\left.\left.\left.\left.\cdot\left\langle I_{D}\left(y_{n}^{\prime}, x ; \tilde{C}_{n-1}\right)\right\rangle_{(n)}\right\rangle_{(n-1)} \cdots\right\rangle_{(3)}\right\rangle_{(2)}\right\rangle_{(1)}\right\rangle_{(0)},
\end{aligned}
$$

where $y_{0}^{\prime}=0$. Also, for $n \geqq 0$,

$$
\Pi_{n}\left(0, y^{\prime}\right)=\sum_{y} p_{y y^{\prime}} g_{n}(0, y)
$$

Finally

$$
\begin{aligned}
R_{N}(0, x)= & \sum_{\left(y_{1}, y_{1}^{\prime}\right)} p_{y_{1} y_{1}^{\prime}} \cdots \sum_{\left.y_{N+1}, y_{N+1}^{\prime}\right)} p_{y_{N+1}, y_{N+1}^{\prime}}\left\langle I\left[0 \circlearrowright y_{1}\right]\right. \\
& \left.\cdot\left\langle I_{D}^{1}\left\langle I_{D}^{2} \cdots\left\langle I_{D}^{N} \cdot\left\{\tau\left(y_{N+1}^{\prime}, x\right)-\tau^{\widetilde{C}_{N}}\left(y_{N+1}^{\prime}, x\right)\right\}\right\rangle_{(N)} \cdots\right\rangle_{(2)}\right\rangle_{(1)}\right\rangle_{(0)}
\end{aligned}
$$

Remark. In the nested expectation

$$
\begin{aligned}
& \left\langle I\left[0 \circlearrowright y_{1}\right]\left\langle I_{D}^{1}\left\langle I_{D}\left(y_{2}^{\prime}, x ; \tilde{C}_{1}\right)\right\rangle_{(2)}\right\rangle_{(1)}\right\rangle_{(0)} \\
= & \left\langle I\left[0 \circlearrowright y_{1}\right]\left\langle I_{D}\left(y_{1}^{\prime}, y_{2} ; \tilde{C}_{0}^{\left\{y_{1}, y_{1}^{\prime}\right\}}(0)\right)\left\langle I_{D}\left(y_{2}^{\prime}, x ; \tilde{C}_{1}^{\left\{y_{2}, y_{2}^{\prime}\right\}}\left(y_{1}^{\prime}\right)\right)\right\rangle_{(2)}\right\rangle_{(1)}\right\rangle_{(0)},
\end{aligned}
$$

which occurs in $g_{2}(0, x)$, it is worth emphasizing again that within the innermost expectation, $\widetilde{C}_{1}^{\left\{y_{2}, y_{2}^{\prime}\right\}}\left(y_{1}^{\prime}\right)$ represents a deterministic set of sites, which is random in the middle expectation. Similarly $\widetilde{C}_{0}^{\left\{y_{1}, y_{1}^{\prime}\right\}}(0)$ is deterministic in the middle expectation, but random in the outer expectation. The situation is entirely analogous for $g_{n}$ and $R_{N}$. 


\subsection{Bounds in $x$-Space for Each Term of the Expansion}

Having completed the derivation of the expansion in the previous section, we now turn to the problem of obtaining bounds on each term in the expansion. We use the van den Berg-Kesten inequality [9] (in the form of (1.33)) as our principal tool, and obtain the bounds given in the following proposition. Before stating the proposition we introduce the following notation:

$$
\begin{gathered}
h_{0}(0, x)=\tau(0, x)^{2}-\delta_{0, x}, \\
h_{n}(0, x)=\sum_{u_{1}, v_{1}} \cdots \sum_{u_{n}, v_{n}} A_{1}\left(0, u_{1}, v_{1}\right) \prod_{i=2}^{n} A_{2}\left(u_{i-1}, v_{i-1}, u_{i}, v_{i}\right) A_{3}\left(u_{n}, v_{n}, x\right), n \geqq 1,
\end{gathered}
$$

where

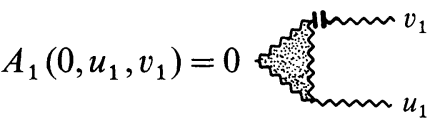

$$
\begin{aligned}
& =\sum_{\left(y_{1}, y_{1}^{\prime}\right)} p_{y_{1} y_{1}^{\prime}} \sum_{z_{1}} \tau\left(0, y_{1}\right) \tau\left(0, z_{1}\right) \tau\left(y_{1}, z_{1}\right) \tau\left(y_{1}^{\prime}, v_{1}\right) \tau\left(z_{1}, u_{1}\right),
\end{aligned}
$$

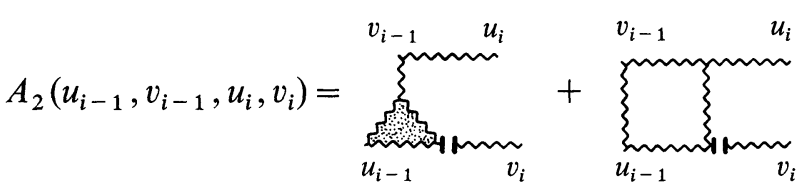

$$
\begin{aligned}
& =\sum_{\left(y_{i}, y_{i}^{\prime}\right)} p_{y_{i} y_{i}^{\prime}} \sum_{z_{i}} \tau\left(u_{i-1}, y_{i}\right) \tau\left(y_{i}, z_{i}\right) \tau\left(z_{i}, u_{i-1}\right) \tau\left(z_{i}, v_{i-1}\right) \tau\left(v_{i-1}, u_{i}\right) \tau\left(y_{i}^{\prime}, v_{i}\right) \\
& +\sum_{\left(y_{i}, y_{i}^{\prime}\right)} p_{y_{i} y_{i}^{\prime}} \sum_{z_{i}} \tau\left(v_{i-1}, u_{i-1}\right) \tau\left(u_{i-1}, y_{i}\right) \tau\left(y_{i}, z_{i}\right) \tau\left(z_{i}, v_{i-1}\right) \tau\left(z_{i}, u_{i}\right) \\
& \cdot \tau\left(y_{i}^{\prime}, v_{i}\right) Z\left(u_{i-1}, v_{i-1}, y_{i}, z_{i}\right) \text {, }
\end{aligned}
$$

where

and

$$
Z(x, y, z, w)= \begin{cases}0 & \text { if } x=y=z=w \\ 1 & \text { otherwise }\end{cases}
$$

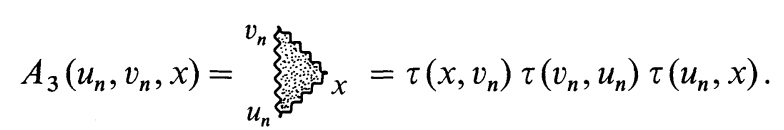

Figure 5 shows the diagrammatic representation for $h_{0}, h_{1}$, and $h_{2}$. The diagrams which occur are closely related to the one particle irreducible Feynman diagrams of a $\varphi^{3}$ field theory. The diagrammatic notation was introduced in Sect. 1.4. In particular, for an unshaded loop the summation over the unlabelled vertices is constrained to disallow the coincidence of all vertices on the loop, whereas a shaded loop has no such constraint. Note that any loop containing one of the summation bonds $\left(y_{i}, y_{i}^{\prime}\right)$ cannot possibly shrink to a point and hence will always be unshaded. With this notation we are now ready to state the bounds on the terms in the expansion of Proposition 2.3. 


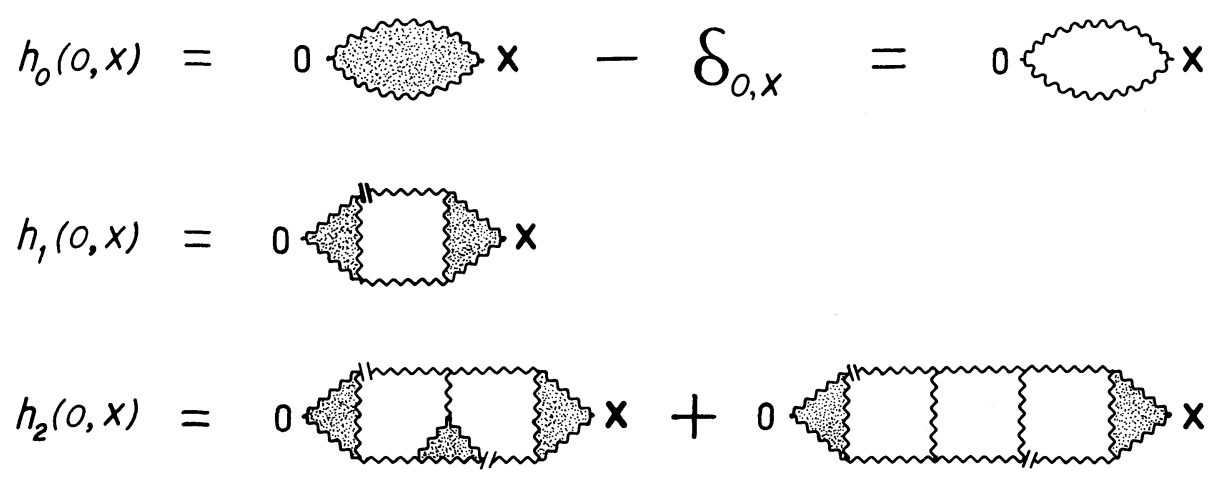

Fig. 5. Diagrammatic representation for $h_{0}, h_{1}$, and $h_{2}$, using the notation introduced in Sect. 1.4

Proposition 2.4. The quantities $g_{n}, \Pi_{n}$ and $R_{N}$ in the expansion (given in Proposition 2.3) for the two-point function $\tau(0, x)$ satisfy the following bounds, for $n, N \geqq 0$ :

$$
\begin{aligned}
& 0 \leqq g_{n}(0, x) \leqq h_{n}(0, x), \\
& 0 \leqq \Pi_{n}\left(0, y^{\prime}\right) \leqq \sum_{y} p_{y y^{\prime}} h_{n}(0, y), \\
& 0 \leqq R_{N}(0, x) \leqq \sum_{\left(y, y^{\prime}\right)} p_{y y^{\prime}} h_{N}(0, y) \tau\left(y^{\prime}, x\right) .
\end{aligned}
$$

Proof. We begin with $g_{0}$ and $\Pi_{0}$. By definition,

$$
g_{0}(0, x)=\operatorname{Prob}(0<x)-\delta_{0, x},
$$

and by the van den Berg-Kesten inequality the right side is less than $\tau(0, x)^{2}-\delta_{0, x}=h_{0}(0, x)$. This is the desired bound on $g_{0}$. Hence, by definition of $\Pi_{0}(2.8 \mathrm{a})$

$$
\Pi_{0}\left(0, y^{\prime}\right) \leqq \sum_{y} p_{y y^{\prime}} h_{0}(0, y) .
$$

This is the desired bound on $\Pi_{0}$. The bound on $R_{0}$ is similar.

To bound $g_{n}, \Pi_{n}$ and $R_{N}$ for $n, N \geqq 1$, we use the following lemma:

Lemma 2.5 .

(a)

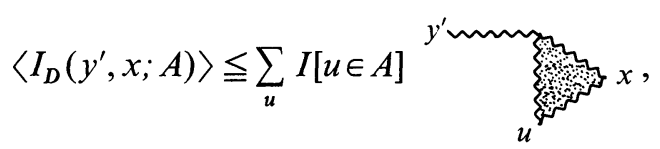

$$
\begin{aligned}
& \left\langle I_{D}\left(y_{i}^{\prime}, y_{i+1} ; A\right) I\left[v \in \tilde{C}^{\left\{y_{i+1}, y_{i+1}^{\prime}\right\}}\left(y_{i}^{\prime}\right)\right]\right\rangle
\end{aligned}
$$

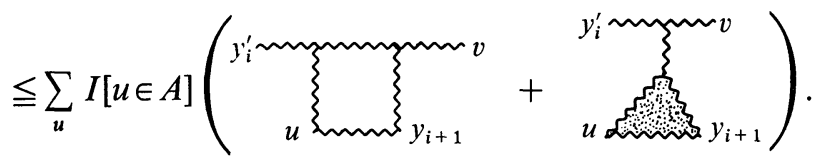

The proof of Lemma 2.5 is deferred to the end of this section. Now we show how the lemma can be used to complete the proof of Proposition 2.4. The basic 
idea is simple: the nested expectations given for $g_{n}, \Pi_{n}$, and $R_{N}$ in Proposition 2.3 are estimated using Lemma 2.5, working from the inside out.

We begin with $g_{n}$, and consider the formula for $g_{n}$ given in Proposition 2.3. We fist estimate the innermost expectation $\left\langle I_{D}\right\rangle_{(n)}$ using Lemma 2.5 (a). (Within this expectation, $\tilde{C}_{n-1}^{\left\{y_{n}, y_{n}^{\prime}\right\}}\left(y_{n-1}^{\prime}\right)$ is deterministic; it is random within the next expectation.) This gives

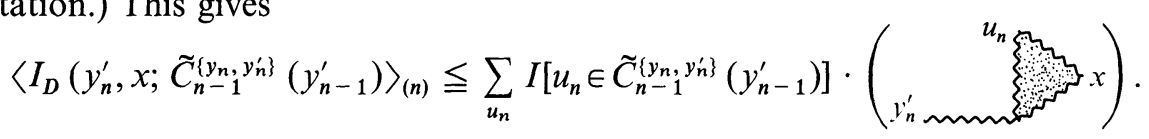

Then we estimate the next expectation (which now contains a factor $I\left[u_{n} \in \tilde{C}_{n-1}^{\left\{y_{n}, y_{n}^{\prime}\right\}}\left(y_{n-1}^{\prime}\right)\right]$ due to the above bound on $\left.\left\langle I_{D}\right\rangle_{(n)}\right)$ using Lemma 2.5(b). This gives

$$
\begin{aligned}
& \left\langle I_{D}\left(y_{n-1}^{\prime}, y_{n} ; \widetilde{C}_{n-2}^{\left\{y_{n-1}, y_{n-1}^{\prime}\right\}}\left(y_{n-2}^{\prime}\right)\right) I\left[u_{n} \in \widetilde{C}_{n-1}^{\left\{y_{n}, y_{n}^{\prime}\right\}}\left(y_{n-1}^{\prime}\right)\right]\right\rangle_{(n-1)}
\end{aligned}
$$

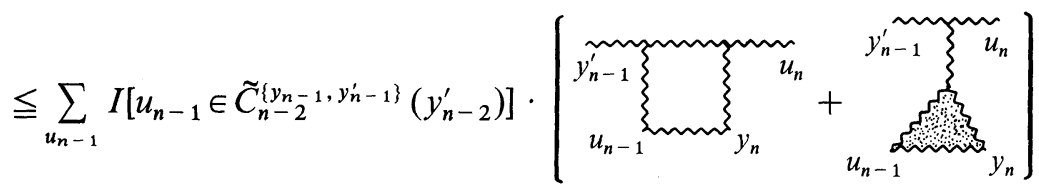

Repeating this process gives the estimate for $g_{n}$ given in Proposition 2.4.

The bound on $\Pi_{n}$ then follows from the fact (Proposition 2.3) that

$$
\Pi_{n}\left(0, y^{\prime}\right)=\sum_{y} p_{y y^{\prime}} g_{n}(0, y)
$$

Finally, we bound $R_{N}$ using the formula given in Proposition 2.3 and the inequality

$$
0 \leqq \tau\left(y^{\prime}, x\right)-\tau^{A}\left(y^{\prime}, x\right) \leqq \tau\left(y^{\prime}, x\right)
$$

to obtain

$$
R_{N}(0, x) \leqq \sum_{y_{N+1}^{\prime}} \Pi_{N}\left(0, y_{N+1}^{\prime}\right) \tau\left(y_{N+1}^{\prime}, x\right) .
$$

This completes the proof of Proposition 2.4, given Lemma 2.5. Now we give the proof of Lemma 2.5.

\section{Proof of Lemma 2.5.}

(a) By definition (Eq. 2.9, see also Fig. 4),

$$
\begin{gathered}
\left\langle I_{D}\left(y^{\prime}, x ; A\right)\right\rangle=\left\langle I\left[y^{\prime} \text { and } x \text { are doubly connected through } A\right]\right\rangle \\
+\left\langleI \left[\exists z \neq y^{\prime} \text { such that } z \text { is connected to } y^{\prime} \text { in } \mathbb{Z}^{d} \backslash A,\right.\right.
\end{gathered}
$$

$z$ is an endpoint of a pivotal bond for the connection of $y^{\prime}$ and $x$, and $z$ is doubly connected to $x$ through $A]>$.

For any bond configuration contributing to the first term on the right side, there exists a site $u \in A$ and three distinct occupied paths connecting $y^{\prime}$ and $x, y^{\prime}$ and $u, u$ and $x$ respectively. Therefore by the van den Berg-Kesten inequality the first term on the right side is bounded above by

$$
\sum_{u \in A} \tau\left(y^{\prime}, u\right) \tau(u, x) \tau\left(x, y^{\prime}\right) .
$$


Furthermore, for any bond configuration contributing to the second term on the right side of (2.19), there is a site $u \in A$ and four distinct paths connecting $y^{\prime}$ and $z, z$ and $x, z$ and $u, u$ and $x$. Note that $z \neq y^{\prime}$. Thus by the van den Berg-Kesten inequality the second term is bounded above by

$$
\sum_{u \in A} \sum_{\substack{z \\ z \neq y^{\prime}}} \tau\left(y^{\prime}, z\right) \tau(z, x) \tau(z, u) \tau(u, x) .
$$

Combining these two gives (a).

(b) By definition of $I_{D}$,

$$
\left\langle I_{D}\left(y_{i}^{\prime}, y_{i+1} ; A\right) I\left[v \in \widetilde{C}^{\left\{y_{t+1}, y_{i+1}^{\prime}\right\}}\left(y_{i}^{\prime}\right)\right]\right\rangle
$$

$\leqq\left\langle I\left[y_{i+1}\right.\right.$ and $y_{i}^{\prime}$ are doubly connected through $A$, and $v$ is connected to $\left.\left.y_{i}^{\prime}\right]\right\rangle$

$+\left\langle I\left[\exists\right.\right.$ a last pivotal bond $\left(z, z^{\prime}\right)$ for the connection from $y_{i}^{\prime}$ to $y_{i+1}, z^{\prime}$ and $y_{i+1}$ are doubly connected through $A, z$ and $y_{i}^{\prime}$ are connected in $\mathbb{Z}^{d} \backslash A$, and $v$ is connected to $\left.y_{i}^{\prime}\right]>$.

We first consider the case $y_{i}^{\prime} \neq y_{i+1}$. For a configuration contributing to the first term on the right side of (2.20), there are sites $u \in A$ and $w \in \mathbb{Z}^{d}$ and five distinct occupied paths joining $y_{i}^{\prime}$ to $u, u$ to $y_{i+1}, y_{i}^{\prime}$ to $w, w$ to $y_{i+1}$, and $w$ to $v$. (Since $y_{i+1}$ and $y_{i}^{\prime}$ are doubly connected through $A$, each path connecting $y_{i}^{\prime}$ to $y_{i+1}$ goes through $A$, and hence $u \in A$ can be chosen such that $w$ and $u$ lie on distinct paths from $y_{i}^{\prime}$ to $y_{i+1}$.) Thus by the van den Berg-Kesten inequality the first term on the right side of (2.20) is bounded above by

$$
\sum_{u \in A}\left(\begin{array}{c}
y_{i}^{\prime} \\
u \\
y_{i+1}
\end{array}\right) .
$$

The second term can be bounded similarly by

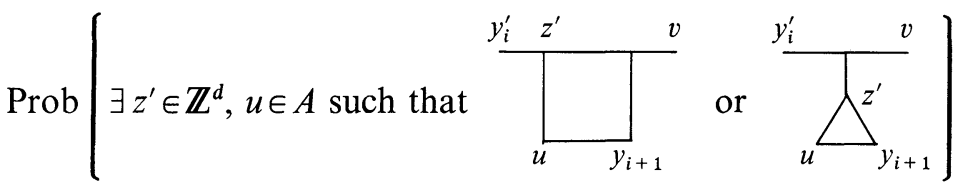

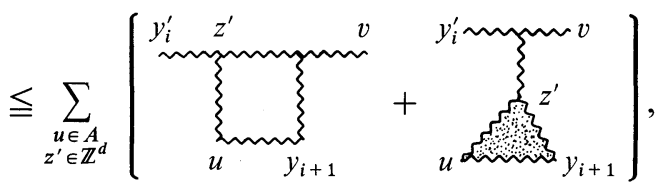

using the van den Berg-Kesten inequality in the last step. In (2.21b), $y_{i}^{\prime} \neq z^{\prime}$, because $z^{\prime}$ is the latter endpoint of a pivotal bond. Combining these two estimates gives (2.17) for the case $y_{i}^{\prime} \neq y_{i+1}$, apart from checking that the loop in the first term on the right side of $(2.21 \mathrm{~b})$ cannot shrink to a point. However, the case where that loop skrinks to a point is already accounted for in the second term of $(2.21 \mathrm{~b})$. (The loop in (2.21 a) cannot shrink to a point when $y_{i}^{\prime} \neq y_{i+1}$.) This proves (2.17) for the case when $y_{i}^{\prime} \neq y_{i+1}$. 
When $y_{i}^{\prime}=y_{i+1}$, it follows from the definition of $I_{D}$ that

$$
\begin{aligned}
& \left\langle I_{D}\left(y_{i}^{\prime}, y_{i+1} ; A\right) I\left[v \in \tilde{C}^{\left\{y_{i+1}, y_{i+1}^{\prime}\right\}}\left(y_{i}^{\prime}\right)\right]\right\rangle \leqq I\left[y_{i}^{\prime} \in A\right]\left\langle I\left[v \in \tilde{C}^{\left\{y_{i+1}, y_{i+1}^{\prime}\right\}}\left(y_{i}^{\prime}\right)\right]\right\rangle \\
& \quad \leqq I\left[y_{i}^{\prime} \in A\right] \operatorname{Prob}\left(y_{i}^{\prime}-v\right) \leqq I\left[y_{i}^{\prime} \in A\right] \tau\left(y_{i}^{\prime}, v\right) .
\end{aligned}
$$

However the upper bound (2.22) is bounded above by the second term in $(2.21 \mathrm{~b})$ (which includes it as the case $y_{i}^{\prime}=z^{\prime}=u=y_{i+1}$ ). Thus (2.17) holds also in the case $y_{i}^{\prime}=y_{i+1}$.

\subsection{Bounds in k-Space for Each Term of the Expansion}

In this section we obtain bounds on $\hat{g}_{n}(k), \hat{\Pi}_{n}(k)$ and $\hat{R}_{N}(k)$ which will be used in Sects. 4 and 5 in deriving the infrared bound. These bounds are given in Proposition 2.6, and follow in a straightforward way from the $x$-space bounds obtained in Sect. 2.2. In the statement of the bounds, derivatives with respect to $k_{\mu}$ are denoted by $\partial_{\mu}$, and $|x|\left(\equiv\|x\|_{2}\right)$ is the Euclidean distance from $x$ to the origin.

Proposition 2.6. The following bounds are satisfied:

$$
\begin{gathered}
\left|\hat{g}_{n}(k)\right| \leqq \sum_{x} h_{n}(0, x), \\
\left|\partial_{\mu}^{s} \hat{g}_{n}(k)\right| \leqq d^{-1} \sum_{x}|x|^{2} h_{n}(0, x), \quad s=1,2, \\
\left|\hat{\Pi}_{n}(k)\right| \leqq \sum_{v} p_{0 v} \sum_{x} h_{n}(0, x), \\
\left|\partial_{\mu}^{s} \hat{\Pi}_{n}(k)\right| \leqq d^{-1}\left[\sum_{v} p_{0 v} \sum_{x}|x|^{2} h_{n}(0, x)+\sum_{v} p_{0 v}|v|^{2} \sum_{x} h_{n}(0, x)\right], \quad s=1,2, \\
0 \leqq \hat{\Pi}_{n}(0)-\hat{\Pi}_{n}(k) \leqq \frac{|k|^{2}}{2 d}\left[\sum_{v} p_{0 v} \sum_{x}|x|^{2} h_{n}(0, x)+\sum_{v} p_{0 v}|v|^{2} \sum_{x} h_{n}(0, x)\right], \\
\left|\hat{R}_{N}(k)\right| \leqq \sum_{v} p_{0 v} \chi(p) \sum_{x} h_{N}(0, x), \\
\left|\partial_{\mu}^{s} \hat{R}_{N}(k)\right| \leqq d^{-1} \chi(p)\left[\sum_{v} p_{0 v} \sum_{x}|x|^{2} h_{N}(0, x)+\sum_{v} p_{0 v}|v|^{2} \sum_{x} h_{N}(0, x)\right] \\
+d^{-1} \sum_{v} p_{0 v} \sum_{x} h_{N}(0, x) \sum_{y}|y|^{2} \tau(0, y), \quad s=1,2 .
\end{gathered}
$$

Proof. These bounds all follow in a straightforward way from the $x$-space bounds given in Proposition 2.4. Beginning first with the bounds which do not involve derivatives, it follows from Proposition 2.4 that

$$
\begin{aligned}
\left|\hat{g}_{n}(k)\right| & =\left|\sum_{x} e^{i k \cdot x} g_{n}(0, x)\right| \leqq \sum_{x} g_{n}(0, x) \leqq \sum_{x} h_{n}(0, x), \\
\left|\hat{\Pi}_{n}(k)\right| & =\left|\sum_{y^{\prime}} e^{i k \cdot y^{\prime}} \Pi_{n}\left(0, y^{\prime}\right)\right| \leqq \sum_{y^{\prime}} \Pi_{n}\left(0, y^{\prime}\right) \leqq \sum_{v} p_{0 v} \sum_{y} h_{n}(0, y),
\end{aligned}
$$


and

$$
\left|\hat{R}_{N}(k)\right|=\left|\sum_{x} e^{i k \cdot x} R_{N}(0, x)\right| \leqq \sum_{x} R_{N}(0, x) \leqq \sum_{v} p_{0 v} \chi(p) \sum_{x} h_{N}(0, x) .
$$

To obtain the bound on $\partial_{\mu}^{s} \hat{g}_{n}(k), s=1,2$, we use symmetry and Proposition 2.4 as follows:

$$
\begin{aligned}
\left|\partial_{\mu}^{s} \hat{g}_{n}(k)\right| & =\left|\sum_{x}\left(i x_{\mu}\right)^{s} e^{i k \cdot x} g_{n}(0, x)\right| \\
& \leqq \sum_{x} x_{\mu}^{2} g_{n}(0, x)=d^{-1} \sum_{x}|x|^{2} g_{n}(0, x) \leqq d^{-1} \sum_{x}|x|^{2} h_{n}(0, x) .
\end{aligned}
$$

The bound on $\partial_{\mu}^{s} \hat{\Pi}_{n}(k)$ is obtained in much the same way. We first note that

$$
\left|\partial_{\mu}^{s} \hat{\Pi}_{n}(k)\right| \leqq d^{-1} \sum_{y^{\prime}}\left|y^{\prime}\right|^{2} \Pi_{n}\left(0, y^{\prime}\right) \leqq d^{-1} \sum_{y, v} p_{0 v}|y+v|^{2} h_{n}(0, y) .
$$

By symmetry the cross term $2 y \cdot v$ gives no contribution to the sum on the right side, and hence

$$
\left|\partial_{\mu}^{s} \hat{\Pi}_{n}(k)\right| \leqq d^{-1} \sum_{y, v} p_{0 v}\left(|y|^{2}+|v|^{2}\right) h_{n}(0, y)
$$

The bounds on $\left|\partial_{\mu}^{s} \hat{R}_{N}(k)\right|$ can be obtained in a similar fashion.

Finally, again using symmetry, we find

$$
\begin{aligned}
\hat{\Pi}_{n}(0)-\hat{\Pi}_{n}(k) & =\sum_{y^{\prime}}\left[1-\cos k \cdot y^{\prime}\right] \Pi_{n}\left(0, y^{\prime}\right) \leqq \frac{1}{2} \sum_{y^{\prime}}\left(\sum_{\mu=1}^{d} k_{\mu} y_{\mu}^{\prime}\right)^{2} \sum_{y} p_{y y^{\prime}} h_{n}(0, y) \\
& =\frac{1}{2} \sum_{\mu, v} k_{\mu} k_{v} \sum_{y, v} p_{0 v}\left(y_{\mu}+v_{\mu}\right)\left(y_{v}+v_{v}\right) h_{n}(0, y) \\
& =\frac{1}{2} \sum_{\mu=1}^{d} k_{\mu}^{2} \sum_{y, v} p_{0 v}\left(\left|y_{\mu}\right|^{2}+\left|v_{\mu}\right|^{2}\right) h_{n}(0, y) \\
& =\frac{|k|^{2}}{2 d} \sum_{y, v} p_{0 v}\left(|y|^{2}+|v|^{2}\right) h_{n}(0, y) .
\end{aligned}
$$

Note that for the nearest neighbour model this expression gives the upper bound

$$
\hat{\Pi}_{n}(0)-\hat{\Pi}_{n}(k) \leqq \frac{\pi^{2}}{4}(1-D(k)) 2 d p \sum_{y}\left(|y|^{2}+1\right) h_{n}(0, y),
$$

since

$$
1-D(k) \equiv \frac{1}{d} \sum_{\mu=1}^{d}\left(1-\cos k_{\mu}\right) \geqq 2 \pi^{-2} \frac{|k|^{2}}{d} .
$$

The corresponding expression for the spread-out models will be given in Sect. 5. It follows immediately from the first equality in (2.23) that the left side is nonnegative.

This completes the proof of Proposition 2.6. 


\section{Diagrammatic Estimates}

In the last section bounds were obtained for $\partial_{\mu}^{s} \hat{g}_{n}(k), \partial_{\mu}^{s} \hat{\Pi}_{n}(k)$ and $\partial_{\mu}^{s} \hat{R}_{N}(k)$, $s=0,1,2$, in terms of $\sum_{x} h_{n}(0, x)$ and $\sum_{x}|x|^{2} h_{n}(0, x)$, for any $\mathbb{Z}^{d}$-invariant model. The definition of $h_{n}(0, x)$ is given in Eqs. (2.10) and (2.11).

In this section we derive upper bounds (also valid in any $\mathbb{Z}^{d}$-invariant model) on $\sum_{x} h_{n}(0, x)$ and $\sum_{x}|x|^{2} h_{n}(0, x)$, in terms of quantities $\bar{T}, \bar{W}$ and $\bar{H}$ which are introduced in the next definition. These quantities will figure in the precise version of (1.18) and for the nearest neighbour model should be thought of as being $O\left(d^{-1}\right)$ uniformly in $p<p_{c}$. Indeed we will prove that such uniform bounds hold in Sect. 4. (Related estimates which show that $\bar{T}, \bar{W}$ and $\bar{H}$ go to zero as $L$ approaches infinity will be obtained in Sect. 5 for the spread-out models.)

Definition 3.1. For $a, a_{1}$, and $a_{2}$ in $\mathbb{Z}^{d}$ we define

$$
\begin{gathered}
T_{a}=\sum_{x, y} \tau(0, x) \tau(x, y) \tau(y, a)-\delta_{a, 0}, \\
W_{a}=\sum_{x}|x|^{2} \tau(0, x) \tau(x, a), \\
H_{a_{1}, a_{2}}=\sum_{x, y, z}|x|^{2} \\
\cdot \tau(x, u) \tau(0, u) \tau(0, z) \tau(u, v) \tau\left(v, y+a_{2}\right) \tau\left(v, z+a_{1}\right) .
\end{gathered}
$$

We write $T_{0}$ and $W_{0}$ simply as $T$ and $W$. We also define

$$
\bar{T}=\sup _{a \in \mathbb{Z}^{d}} T_{a}, \bar{W}=\sup _{a \in \mathbb{Z}^{d}} W_{a}, \bar{H}=\sup _{a_{1}, a_{2} \in \mathbb{Z}^{d}} H_{a_{1}, a_{2}} .
$$

Finally we define

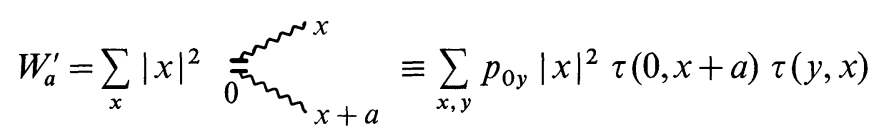

and

$$
\bar{W}^{\prime}=\sup _{a} W_{a}^{\prime}
$$

We simply write $W^{\prime}$ for $W_{0}^{\prime}$.

In Sects. 3.1 and 3.2 the following lemma is proved.

\section{Lemma 3.2.}

$$
0 \leqq \sum_{x} h_{0}(0, x) \leqq \frac{T}{3}
$$

and for $n \geqq 1$,

$$
0 \leqq \sum_{x} h_{n}(0, x) \leqq(1+T)^{2}\left[\sum_{v} p_{0 v} \frac{T}{3}+2\left(\sum_{v} p_{0 v}^{2} T / 3\right)^{1 / 2}+2 \bar{p}\right] r^{n-1},
$$


where

$$
\bar{p}=\sup _{v} p_{0 v}
$$

and

$$
r=(1+T+\bar{T})\left[\bar{p}+\sum_{v} p_{0 v} \bar{T}\right]
$$

$$
\begin{gathered}
0 \leqq \sum_{x}|x|^{2} h_{0}(0, x) \leqq W \\
0 \leqq \sum_{x}|x|^{2} h_{1}(0, x) \leqq W^{\prime}+6 T(1+T) \bar{W}^{\prime}+6 \bar{W}(1+T)\left[\bar{p}+\sum_{v} p_{0 v} \bar{T}\right]
\end{gathered}
$$

and for $n \leqq 1$,

$$
\begin{aligned}
0 \leqq & \sum_{x}|x|^{2} h_{n}(0, x) \leqq(2 n+1)\left(\frac{n}{2} \sum_{v} p_{0 v}+(n+1) r\right)(1+T)^{2} r^{n-1} \bar{W} \\
& +(2 n+1) \llbracket \frac{n}{2} \rrbracket(1+T)^{2} r^{n-1} \bar{W}^{\prime}+(2 n+1) \llbracket \frac{n-1}{2} \rrbracket(1+T)^{2} r^{n-2} \bar{H}\left(\sum_{v} p_{0 v}\right)^{2},
\end{aligned}
$$

where $\llbracket x \rrbracket$ denotes the largest integer which does not exceed $x$.

Remark. The exact form of the upper bounds in the lemma is not important unless a good value of $d_{0}$ is sought. Otherwise the relevant feature, for the nearestneighbour model, of these bounds is that under the assumption that $\bar{T}, \bar{W}, \bar{W}^{\prime}, \bar{H}$ are all $O\left(d^{-1}\right)$ and $2 d p$ is $O(1)$, the bounds imply

$$
\begin{gathered}
0 \leqq \sum_{x} h_{n}(0, x) \leqq\left\{\begin{array}{ll}
O\left(d^{-1}\right) & n=0 \\
O\left(d^{-1}\right)^{n} & n=1,2, \ldots
\end{array}\right. \text { and } \\
0 \leqq \sum_{x}|x|^{2} h_{n}(0, x) \leqq \begin{cases}O\left(d^{-1}\right) & n=0,1 \\
n^{2} O\left(d^{-1}\right)^{n-1} & n=2,3,4, \ldots\end{cases}
\end{gathered}
$$

(The $n \geqq 2$ bound in (b) is believed not to be optimal, but it does suffice for our purposes.) An analogous remark applies for the spread-out models, as described in Sect. 5.

The proof of Lemma 3.2(a) is given in Sect. 3.1 and the proof of part (b) in Sect. 3.2. Finally in Sect. 3.3 we prove the following bounds on $T_{a}$ and $W_{a}^{\prime}$ :

\section{Lemma 3.3.}

$$
\begin{gathered}
T_{a} \leqq T+(3 T / 2 d)^{1 / 2} \\
W^{\prime} \equiv W_{0}^{\prime} \leqq \sum_{v} p_{0 v} W+\sum_{v} p_{0 v}|v|(W T / 3)^{1 / 2}+\left(\sum_{v} p_{0 v}^{2}|v|^{2} W\right)^{1 / 2} \\
W_{a}^{\prime} \leqq 2 \sum_{v} p_{0 v} \bar{W}+2 \sum_{v} p_{0 v}|v|^{2} \frac{T}{3}+4\left(\sum_{v} p_{0 v}^{2}|v|^{4} \frac{T}{3}\right)^{1 / 2}+4 p_{0 a}|a|^{2} .
\end{gathered}
$$

\subsection{Bounds on $\sum_{x} h_{n}(0, x)$}

In this and the next section we make extensive use of the graphical notation introduced in Sect.1.4 and the graphical representation for $h_{n}$ given in Eqs. 
(2.11-14) and Fig. 5. This section is devoted to the proof of Lemma 3.2(a). The proof uses translation invariance and repeated application of the simple inequality

$$
\left|\sum_{x} f(x) g(x)\right| \leqq \sup _{x}|f(x)| \sum_{x}|g(x)| .
$$

This inequality is used to reduce $\sum_{x} h_{n}(0, \mathrm{x})$ to a product of basic units.

The case $n=0$ is the simplest. We just observe that

$$
T=\sum_{x, y} \tau(0, x) \tau(x, y) \tau(y, 0)-1 \geqq 3 \sum_{x \neq 0} \tau(0, x) \tau(x, 0),
$$

and hence

$$
\sum_{x} h_{0}(0, x)=\sum_{x \neq 0} \tau(0, x)^{2} \leqq \frac{T}{3} .
$$

For $n \geqq 1$ we use translation invariance to rewrite (2.11) as

$$
\sum_{x} h_{n}(0, x)=\sum_{a_{1}, \ldots, a_{n}} \tilde{A}_{1}\left(a_{1}\right) \prod_{i=2}^{n} \tilde{A}_{2}\left(a_{i-1}, a_{i}\right) \tilde{A}_{3}\left(a_{n}\right)
$$

where

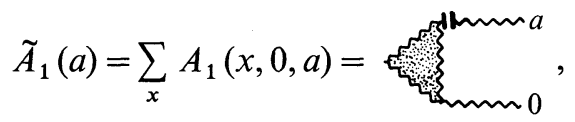

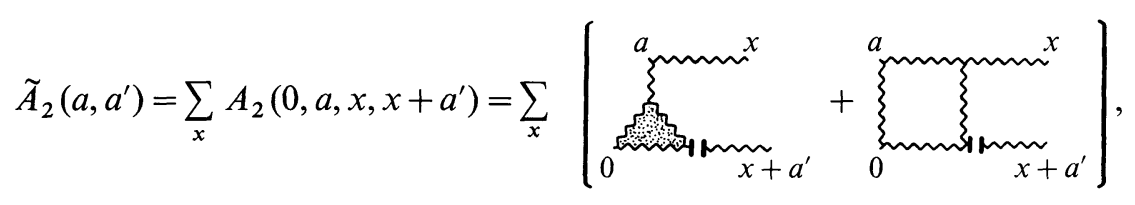

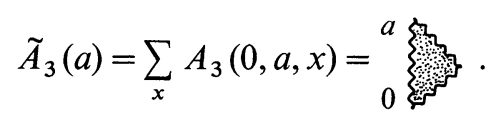

Then we apply (3.5) repeatedly to obtain

$$
\sum_{x} h_{n}(0, x) \leqq\left(\sup _{a} \tilde{A}_{1}(a)\right)\left(\sup _{a^{\prime}}\left\{\sum_{a} \tilde{A}_{2}\left(a, a^{\prime}\right)\right\}\right)^{n-1}\left(\sum_{a} \tilde{A}_{3}(a)\right) .
$$

The last factor on the right side of (3.8) is $1+T$. To complete the proof of Lemma 3.2(a) we use (3.8) and the following lemma.

Lemma 3.4. The following inequalities hold for any $a \in \mathbb{Z}^{d}$ :

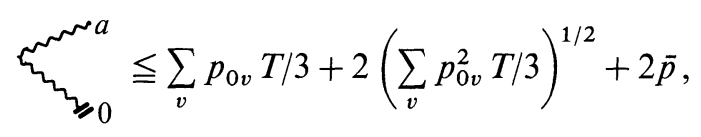

$$
\sum_{v} \leqq \sum_{v} p_{0 v} \bar{T}+\bar{p},
$$


(c)

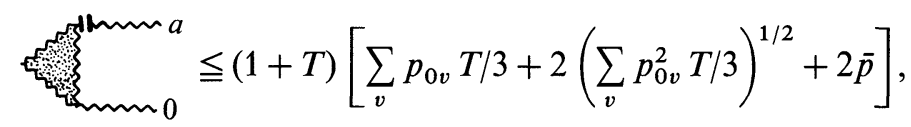

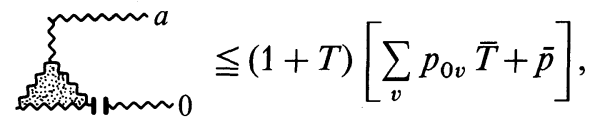

$$
\sum_{\operatorname{minn} 0} \leqq \bar{T}\left[\sum_{v} p_{0 v} \bar{T}+\bar{p}\right]
$$

Proof. (a) Using the Schwarz inequality and the inequality used to bound $\sum_{x} h_{0}(0, x)$ we obtain

$$
\begin{aligned}
\sum_{y=0}^{\sim \sim a} a & \equiv \sum_{x, v} p_{0 v} \tau(v, x) \tau(x, a)=\sum_{v} p_{0 v}\left[\sum_{x \neq v, a} \tau(v, x) \tau(x, a)+2 \tau(v, a)\right] \\
& \leqq \sum_{v} p_{0 v} T / 3+2 \sum_{v \neq a} p_{0 v} \tau(v, a)+2 p_{0 a} \\
& \leqq \sum_{v} p_{0 v} T / 3+2\left(\sum_{v} p_{0 v}^{2} T / 3\right)^{1 / 2}+2 \bar{p} .
\end{aligned}
$$

(b) Using translation invariance the left side is equal to

$$
\sum_{v} p_{0 v} \sum_{x, y} \tau(-v, x) \tau(x, y) \tau(y, a) .
$$

By considering separately in the sum over $v$ the term $v=-a$, we obtain the upper bound

$$
\sum_{v \neq a} p_{0 v} \bar{T}+p_{0 a}(1+T) \leqq \sum_{v} p_{0 v} \bar{T}+\bar{p}
$$

(c) By $(3,5)$,

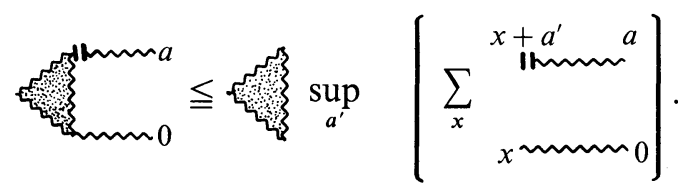

The first factor on the right side is equal to $1+T$, and the second can be bounded using (a) and translation invariance.

(d) and (e) are proved similarly.

Now the desired bound on $\sum_{x} h_{n}(0, x)$ follows immediately from (3.8) and (c-e), and the proof of Lemma 3.2 (a) is complete. 


\subsection{Bounds on $\sum_{x}|x|^{2} h_{n}(0, x)$}

In this section we prove Lemma 3.2(b). The proof is similar to the proof of Lemma 3.2 (a) given in the last section, although the situation here is more complicated due to the presence of the factor $|x|^{2}$.

The case $n=0$ is the simplest, since by definition of $W$

$$
\sum_{x}|x|^{2} h_{0}(0, x)=\sum_{x}|x|^{2} \tau(0, x)^{2}=W .
$$

For higher order terms the basic idea is to use (3.5) together with the triangle inequality, as we now illustrate for the case $n=1$. By definition of $h_{1}$,

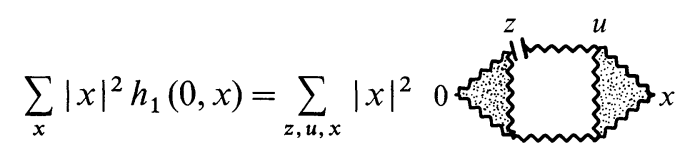

and by the triangle inequality

$$
|x|^{2} \leqq(|x-u|+|u-z|+|z|)^{2} \leqq 3\left(|x-u|^{2}+|u-z|^{2}+|z|^{2}\right) .
$$

We insert (3.10) into (3.9) and consider separately the case where both of the shaded triangles in (3.9) are points. In this special case (3.9) contributes $W^{\prime}$, and hence

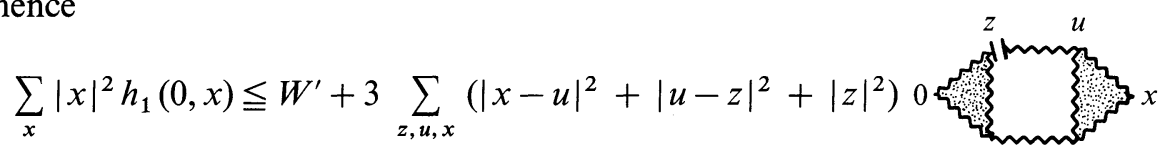

where at least one of the shaded triangles on the right side is not a point. Each of the three terms in the summation on the right side is now treated separately, using (3.5), translation invariance and Lemma 3.4. For example,

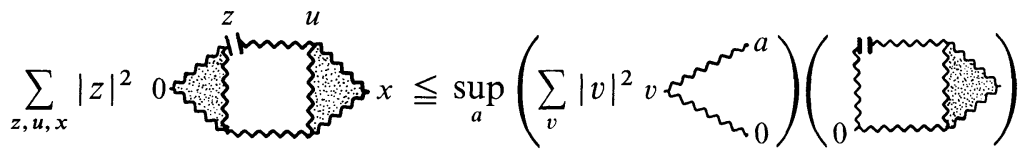

$$
\begin{aligned}
& \leqq \bar{W} \sup _{a}\left(\xi_{\operatorname{mann} a}^{a}\right)(1+T) \leqq \bar{W}\left[\sum_{v} p_{0 v} \bar{T}+\bar{p}\right](1+T) .
\end{aligned}
$$

The term involving $|x-u|^{2}$ is handled similarly, and satisfies the same upper bound. The remaining term is

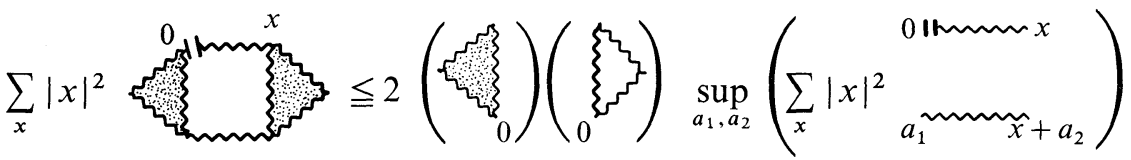

$$
\begin{aligned}
& \leqq 2(1+T) T \bar{W}^{\prime},
\end{aligned}
$$


where we have used the fact that one of the two triangles is not a point. As a result,

$$
\sum_{x}|x|^{2} h_{1}(0, x) \leqq W^{\prime}+6 T(1+T) \bar{W}^{\prime}+6 \bar{W}(1+T)\left[\sum_{v} p_{0 v} \bar{T}+\bar{p}\right],
$$

which is the desired bound.

The case $n \geqq 2$ can be handled in a similar fashion, although the additional factors of $\tilde{A}_{2}$ make the analysis more involved. It is at this stage that it becomes necessary to work with the quantity $\bar{H}$, as we are unable to bound the diagrams encountered solely in terms of $\bar{T}$ and $\bar{W}$.

It is convenient to use the following expression for $h_{n}(0, x)$, which is equivalent to (2.11). To write the expression we recall the definition of $A_{3}(u, v, x)$ in (2.14) and define

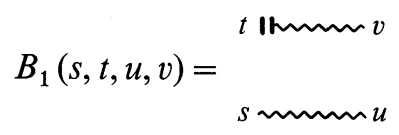

and

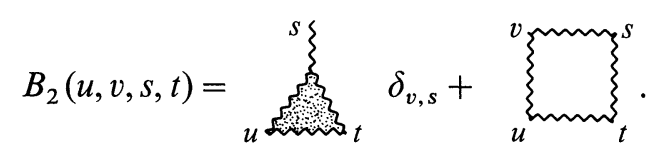

Then (2.11) can be rewritten

$$
\begin{aligned}
h_{n}(0, x) & =\sum_{\substack{s_{k}, t_{k} \\
u_{k}, v_{k}}} A_{3}\left(0, s_{1}, t_{1}\right) \prod_{i=1}^{n} B_{1}\left(s_{i}, t_{i}, u_{i}, v_{i}\right) \prod_{i=2}^{n} B_{2}\left(u_{i-1}, v_{i-1}, s_{i}, t_{i}\right) A_{3}\left(u_{n}, v_{n}, x\right) \\
& \equiv \sum h_{n}(0, \vec{s}, \vec{t}, \vec{u}, \vec{v}, x) .
\end{aligned}
$$

To bound $\sum_{x}|x|^{2} h_{n}(0, x)$ we use the triangle inequality as for the case $n=1$ :

$$
\begin{aligned}
|x|^{2} \leqq & (2 n+1)\left(\left|t_{1}\right|^{2}+\left|v_{1}-t_{1}\right|^{2}+\left|s_{2}-v_{1}\right|^{2}+\ldots\right. \\
& \left.+\left\{\left[\left|s_{n}-u_{n}\right|^{2}+\left|u_{n}-x\right|^{2}\right] \text { or }\left[\left|t_{n}-v_{n}\right|^{2}+\left|v_{n}-x\right|^{2}\right]\right\}\right),
\end{aligned}
$$

where in the term in brace brackets in (3.14) the first expression is used if $n$ is even and the second if $n$ is odd.

The effect of each term on the right side of (3.14) is treated separately, and we proceed term by term. Beginning with the $\left|t_{1}\right|^{2}$ term, and using an unlabelled sum to denote summation over all vertices, a minor change in the organization gives

$$
\begin{aligned}
& (2 n+1)^{-1} \sum\left|t_{1}\right|^{2} h_{n}(0, \vec{s}, \vec{t}, \vec{u}, \vec{v}, x)
\end{aligned}
$$

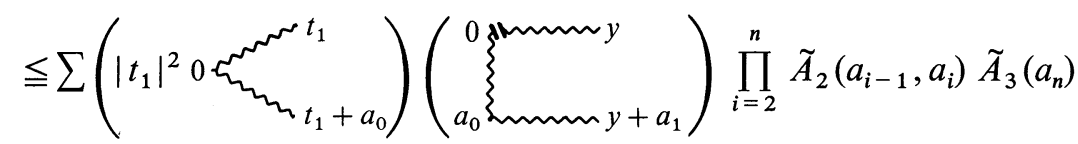

$$
\begin{aligned}
& \leqq \bar{W} \sup _{a_{1}}\left(\sum_{\operatorname{man} a_{1}}^{\sin 0}\right)\left(\sup _{a^{\prime}} \sum_{a} \tilde{A}_{2}\left(a, a^{\prime}\right)\right)^{n-1} \sum_{a} \tilde{A}_{3}(a) .
\end{aligned}
$$

Now Lemma 3.4 applies as before. 
The $\left|v_{1}-t_{1}\right|^{2}$ term is treated as follows:

$$
\begin{aligned}
& (2 n+1)^{-1} \sum\left|v_{1}-t_{1}\right|^{2} h_{n}(0, \vec{s}, \vec{t}, \vec{u}, \vec{v}, x)
\end{aligned}
$$

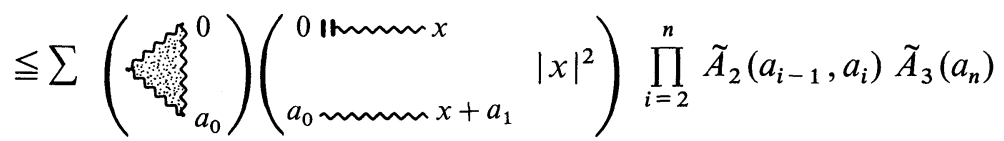

$$
\begin{aligned}
& \leqq \sum_{a_{0}} \tilde{A}_{3}\left(a_{0}\right) \bar{W}^{\prime}\left(\sup _{a^{\prime}} \sum_{a} \tilde{A}_{2}\left(a, a^{\prime}\right)\right)^{n-1} \sum_{a} \tilde{A}_{3}(a) \text {, }
\end{aligned}
$$

and again Lemma 3.4 can be applied.

The term involving $\left|s_{2}-v_{1}\right|^{2}$ can be organized as follows:

$$
\begin{aligned}
& (2 n+1)^{-1} \sum\left|s_{2}-v_{1}\right|^{2} h_{n}(0, \vec{s}, \vec{t}, \vec{u}, \vec{v}, x)
\end{aligned}
$$

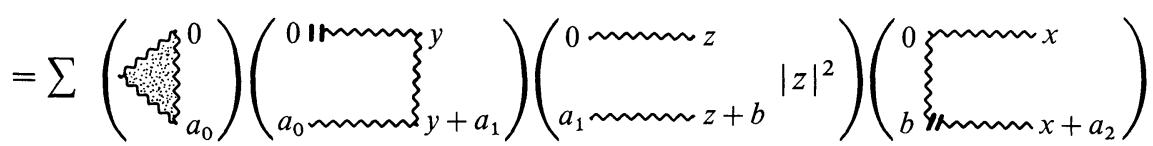

$$
\begin{aligned}
& \cdot \prod_{i=3}^{n} \tilde{A}_{2}\left(a_{i-1}, a_{i}\right) \tilde{A}_{3}\left(a_{n}\right)
\end{aligned}
$$

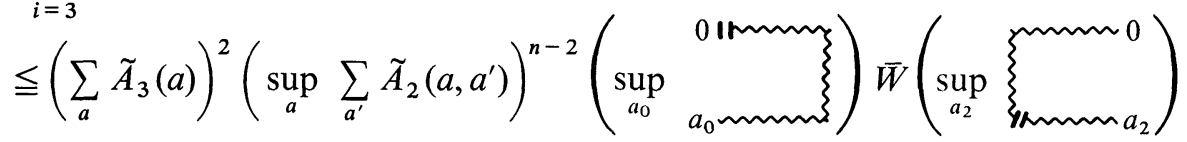

Now Lemma 3.4 can be applied.

To estimate the term involving $\left|u_{2}-s_{2}\right|^{2}$ we define

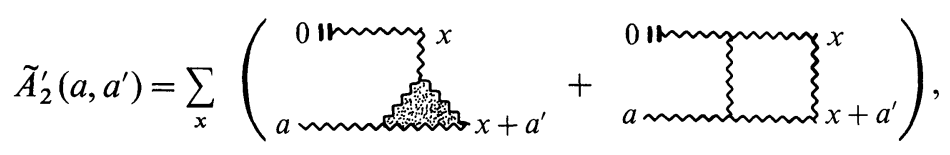

and write

$$
\begin{aligned}
& (2 n+1)^{-1} \sum\left|u_{2}-s_{2}\right|^{2} h_{n}(0, \vec{s}, \vec{t}, \vec{u}, \vec{v}, x) \\
& \quad \leqq \sum \tilde{A}_{3}\left(a_{0}\right) \tilde{A}_{2}^{\prime}\left(a_{0}, a_{1}\right)\left(\begin{array}{c}
0 \operatorname{man} x \\
a_{1} \operatorname{\operatorname {manm}} a_{2}+x
\end{array}\right) \prod_{i=3}^{n} \tilde{A}_{2}\left(a_{i-1}, a_{i}\right) \tilde{A}_{3}\left(a_{n}\right) \\
& \leqq \bar{W} \sum_{v} p_{0 v}\left(\sum_{a} \tilde{A}_{3}(a)\right)^{2}\left(\sup _{a} \sum_{a^{\prime}} \tilde{A}_{2}^{\prime}\left(a, a^{\prime}\right)\right)\left(\sup _{a^{\prime}} \sum_{a} \tilde{A}_{2}\left(a, a^{\prime}\right)\right)^{n-2}
\end{aligned}
$$

Now Lemma 3.4 can be applied.

Finally we treat the term $\left|u_{2}-t_{3}\right|^{2}$, which is the most complicated one, and in which we use $\bar{H}$. The contribution due to the term involving the second term on the right side of (3.12) is equal to

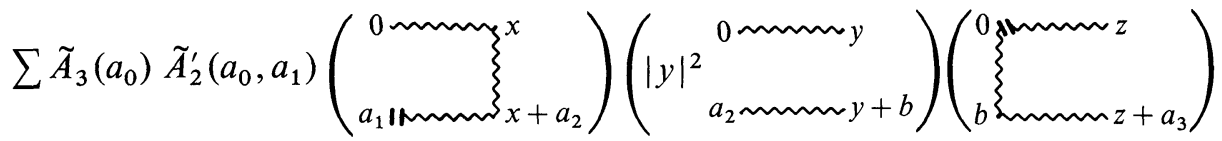

$$
\begin{aligned}
& \cdot \prod_{i=4}^{n} \tilde{A}_{2}\left(a_{i-1}, a_{i}\right) \tilde{A}_{3}\left(a_{n}\right) \text {, }
\end{aligned}
$$


which can be bounded above as was done for the $\left|s_{2}-v_{1}\right|^{2}$ term. The contribution due to the first term on the right side of (3.12) is equal to

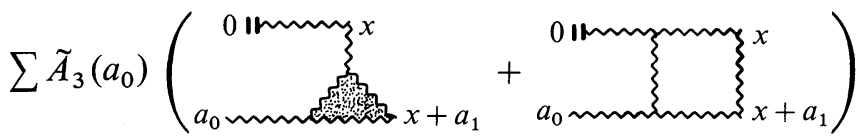

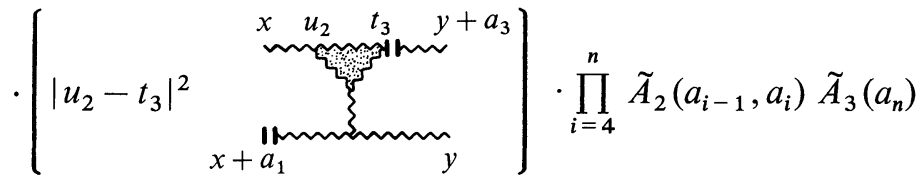

$$
\begin{aligned}
& \leqq \bar{H}\left(\sum_{v} p_{0 v}\right)^{2}\left(\sum_{a} \tilde{A}_{3}(a)\right)^{2}\left(\sup _{a} \sum_{a^{\prime}} \tilde{A}_{2}^{\prime}\left(a, a^{\prime}\right)\right)\left(\sup _{a^{\prime}} \sum_{a} \tilde{A}_{2}\left(a, a^{\prime}\right)\right)^{n-3} \text {, }
\end{aligned}
$$

and again Lemma 3.4 can be applied.

This illustrates the method for bounding $\sum_{x}|x|^{2} h_{n}(0, x)$. By applying Lemma 3.4 and carefully counting the number of terms of each type which occurs, the upper bound in the statement of the lemma can be obtained. We omit the straightforward but tedious details.

\subsection{Proof of Lemma 3.3}

In this section we prove Lemma 3.3. We begin with the inequality for $T_{a}$. Fix $a \neq 0$. Using the fact that $\hat{\tau}(k) \geqq 0[6]$,

$$
\begin{aligned}
T_{a} & =(2 \pi)^{-d} \int d^{d} k\left[\hat{\tau}(k)^{3}-1\right] e^{i k \cdot a} \\
& =(2 \pi)^{-d} \int d^{d} k[\hat{\tau}(k)-1]^{2}[\hat{\tau}(k)+2] e^{i k \cdot a}+3 \tau(0, a) \\
& \leqq(2 \pi)^{-d} \int d^{d} k[\hat{\tau}(k)-1]^{2}[\hat{\tau}(k)+2]+3 \tau(0, a)=T+3 \tau(0, a) .
\end{aligned}
$$

Now for $a \neq 0$ it follows by symmetry that there are at least $2 d$ terms which give the same contributions as $\tau(0, a)^{2}$ in

$$
B \equiv \sum_{x \neq 0} \tau(0, x)^{2} \leqq T / 3 .
$$

This leads to the desired inequality for $T_{a}$.

To obtain the inequality for $W^{\prime}$ we argue using the Schwarz inequality as follows:

$$
\begin{aligned}
W^{\prime} & \equiv \sum_{x, y} p_{0 y}|x|^{2} \tau(0, x) \tau(x, y) \leqq \sum_{y} p_{0 y} \sum_{x} \tau(0, x) \tau(x, y)[|x||x-y|+|x||y|] \\
& \leqq \sum_{y} p_{0 y} W+\sum_{y} p_{0 y}\left(\sum_{x \neq y} \tau(0, x) \tau(y, x)|x||y|+\tau(0, y)|y|^{2}\right) \\
& =\sum_{y} p_{0 y} W+\sum_{y} p_{0 y}|y| \sum_{x \neq y} \tau(0, x)|x| \tau(x, y)+\sum_{y} p_{0 y}|y||y| \tau(0, y) \\
& \leqq \sum_{y} p_{0 y} W+\sum_{y} p_{0 y}|y|(W B)^{1 / 2}+\left(\sum_{y} p_{0 y}^{2}|y|^{2} W\right)^{1 / 2} .
\end{aligned}
$$


Finally, the bound on $W_{a}^{\prime}$ is obtained similarly:

$$
\begin{aligned}
W_{a}^{\prime} \equiv & \sum_{x, y} p_{0 y}|x|^{2} \tau(y, x) \tau(0, x+a) \\
\leqq & 2 \sum_{x, y} p_{0 y} \tau(y, x) \tau(0, x+a)\left[|x-y|^{2}+|y|^{2}\right] \\
= & 2 \sum_{x, y} p_{0 y} \tau(0, x) \tau(0, x+a+y)\left[|x|^{2}+|y|^{2}\right] \\
= & 2 \sum_{y} p_{0 y} \sum_{x} \tau(0, x) \tau(0, x+a+y)|x|^{2} \\
& +2 \sum_{y} p_{0 y}|y|^{2}\left[\sum_{x \neq 0,-y-a} \tau(0, x) \tau(0, x+a+y)+2 \tau(a, y)\right] \\
\leqq & 2 \sum_{y} p_{0 y} \bar{W}+2 \sum_{y} p_{0 y}|y|^{2} B+4 \sum_{y \neq a} p_{0 y}|y|^{2} \tau(a, y)+4 p_{0 a}|a|^{2} \\
\leqq & 2 \sum_{y} p_{0 y} \bar{W}+2 B \sum_{y} p_{0 y}|y|^{2}+4\left(B \sum_{y \neq a} p_{0 y}^{2}|y|^{4}\right)^{1 / 2}+4 p_{0 a}|a|^{2} .
\end{aligned}
$$

The desired bound now follows from the inequality $B \leqq T / 3$.

\section{Proof of the Main Results (Theorem 1.1) for the Nearest-Neighbour Model}

In this section we use the results of Sect. 2 and 3 to prove Theorem 1.1. The general structure of the proof follows the outline given in Sect.1.3 and is described in Sect. 4.1. Throughout this section we consider primarily the nearest-neighbour model, for which $p_{0 v}=p$ if $|v|=1$ and $p_{0 v}=0$ otherwise. In particular, $\sum_{v} p_{0 v}=2 d p$. We use this and similar facts in the bounds given in Lemma 3.2, without further mention. However since Lemmas 4.1 and 4.2 hold quite generally, we state and prove these lemmas for both the nearest-neighbour and the spreadout models introduced in Sect.1.1.

We use the notation

$$
D_{L}(k)=\sum_{x} p_{0 x} e^{i k \cdot x}
$$

for the spread-out models at the gaussian critical value $p_{G}$, i.e., the value of $p$ for which $D_{L}(0)=1$. For the nearest neighbour model at its critical value $p_{G}=1 / 2 d$ we write simply

$$
D(k)=d^{-1} \sum_{\mu} \cos k_{\mu}
$$

in place of $D_{L}(k)$, and we use $D_{G}$ to represent either $D$ or $D_{L}$.

\subsection{General Structure}

The fact that the triangle diagram $\nabla(p)$ is bounded uniformly in $p<p_{c}$ is proved using the following three results. Lemmas 4.1 and 4.2 are valid for both the nearest neighbour model and the spread-out models introduced in Sect.1.1. Proposi- 
tion 4.3 is valid as stated only for the nearest-neighbour model; a variation valid for the spread-out models is proved in Sect. 5.1.

Lemma 4.1. For both the models (i) and (ii) of Sect.1.1, if $0 \leqq p \leqq p_{G}$ then $\tau_{p}(x, y) \leqq C_{G}(x, y)$, where $C_{G}(x, y)$ denotes the gaussian propagator at $p=p_{G}$ :

$$
C_{G}(x, y)=(2 \pi)^{-d} \int d^{d} k \frac{e^{i k \cdot(y-x)}}{1-D_{G}(k)} .
$$

Lemma 4.2. For both models (i) and (ii) of Sect.1.1, T, $W_{a}$, and $H_{a_{1}, a_{2}}$ are continuous in $p$, for $p<p_{c}$ and for all $a, a_{1}, a_{2} \in \mathbb{Z}^{d}$.

Proposition 4.3. For the nearest-neighbour model (i), there is a universal constant $d_{0}>6$ such that for $d \geqq d_{0}$ and for any fixed $p \in\left[1 / 2 d, p_{c}\right), P_{4} \Rightarrow P_{3}$, where $P_{\alpha}$ is the statement that the following set of inequalities holds:

$$
\begin{gathered}
T \leqq \alpha K_{T} d^{-1}, \quad W \leqq \alpha K_{W} d^{-1}, \quad 2 d p \leqq \alpha \\
W_{a} \leqq \alpha K_{W}^{\prime} d^{-1} \text { for }\|a\|_{1} \leqq 2 \chi(p)\{(d+2) \ln [5 \chi(p)]+2 \ln d\} \\
H_{a_{1}, a_{2}} \leqq 10 \alpha K_{W} d^{-1} \text { for } \max _{i=1,2}\left\|a_{i}\right\|_{1} \leqq 2 \chi(p)\{(5 d+2) \ln [5 \chi(p)]+2 \ln d\}
\end{gathered}
$$

In Proposition $4.3 K_{T}$ and $K_{W}$ are universal constants such that for $d \geqq 7$,

$$
T_{G} \equiv \sum_{x, y} C(0, x) C(x, y) C(y, 0)-C(0,0)^{3} \leqq \frac{1}{25} K_{T} d^{-1},
$$

and

$$
W_{G} \equiv \sum_{x}|x|^{2} C(0, x)^{2} \leqq \frac{1}{30} K_{W} d^{-1} .
$$

The existence of such constants follows from Appendix B of [20] or Lemma 3.1 of [31]. (In the notation of [31], $C(0, x)=\sum_{T=0}^{\infty} N_{0}(x, T)$.) The constant $K_{W}^{\prime}$ is a (large) universal constant which depends only on $K_{T}$ and $K_{W}$ and is determined in the proof of the proposition. The statement $P_{\alpha}$, for fixed $p<p_{c}$, involves only finitely many inequalities, since $\chi(p)<\infty$. For $\|a\|_{1}$ or $\left\|a_{i}\right\|_{1}$ violating the inequalities in $P_{\alpha}$, it follows from the exponential decay of $\tau_{p}(x, y)$ that $W_{a} \leqq d^{-1}$ and $H_{a_{1}, a_{2}} \leqq d^{-1}$; see the proof of Lemma 4.4.

It follows immediately from Lemma 4.1 that for $p \in[0,1 / 2 d]$ the first three inequalities in the definition of $P_{\alpha}$ hold with $\alpha=1$. The other inequalities in the definition of $P_{1}$ can be proved for $p \in[0,1 / 2 d]$ by using Lemma 4.1 to bound all propagators by the gaussian propagator, and then analyzing the resulting gaussian quantities. This can be done using the method of Sect.4.3.3(d-e), by putting $\hat{G}=1$ and $\hat{\Pi}=0$ there. It follows from the fact that $P_{1}$ holds for $p \leqq 1 / 2 d$, together with Lemma 4.2 and Proposition 4.3 that there are forbidden regions in the graphs of $T, W, W_{a}$ and $H_{a_{1}, a_{2}}$ as a function of $p$, for $p<p_{c}$, as illustrated in Fig. 1. Therefore $P_{3}$ holds and so in particular $\nabla(p)=T(p)+1$ is bounded 
uniformly in $p<p_{c}$. Since $\tau_{p}(x, y)$ is an increasing and continuous function of $p$ [5], it then follows from the monotone convergence theorem that

$$
\nabla\left(p_{c}\right)=\lim _{p \uparrow p_{c}} \nabla(p) \leqq 3 K_{T} d^{-1}+1,
$$

and hence the triangle condition is satisfied.

In the course of the proof of Proposition 4.3 it is shown that if $P_{4}$ holds, then the infrared bound stated in Theorem 1.1 follows. But as we have noted it follows from Lemmas 4.1 and 4.2 and Proposition 4.3 that in fact $P_{3}$ holds, and hence the weaker statement $P_{4}$ holds, and the infrared bound follows. Also, it follows almost automatically from the proof that the relation $\xi_{2}(p)^{2} \sim \chi(p)$ holds, and hence $v_{2}=1 / 2$.

The proof of Theorem 1.1 has now been reduced to proving Lemmas 4.1 and 4.2 and Proposition 4.3, and showing that the infrared bound follows from $P_{4}$. This is done in the remaining subsections. The two lemmas are elementary and do not use the expansion. The proof of Proposition 4.3 is the significant part of the analysis.

\subsection{Proof of Lemmas 4.1 and 4.2}

In this section we prove Lemmas 4.1 and 4.2.

Proof of Lemma 4.1. Fix any $p \in\left[0, p_{G}\right]$. Any bond configuration in which $x$ and $y$ are connected contains an occupied path $\omega$ from $x$ to $y$. We denote the sum over all paths from $x$ to $y$ by $\sum_{\omega: x \rightarrow y}^{\prime}$, and the sum over all simple (not necessarily selfavoiding) walks from $x$ to $y$, consisting of bonds for which $p_{b} \neq 0$, by $\sum_{\omega: x \rightarrow y}$. Then

$$
\begin{aligned}
\tau_{p}(x, y) & \leqq \sum_{\omega: x \rightarrow y}^{\prime} \operatorname{Prob}_{p}(\omega \text { is occupied })=\sum_{\omega: x \rightarrow y}^{\prime} \prod_{b \in \omega} p_{b} \\
& \leqq \sum_{\omega: x \rightarrow y} \prod_{b \in \omega} p_{b} \leqq C_{G}(x, y),
\end{aligned}
$$

where in the last inequality we used $p \leqq p_{G}$.

Proof of Lemma 4.2. In [18] it was proved that for quite general finite range models, if $\chi(p)<\infty$ then $\tau_{p}(x, y)$ decays exponentially. Moreover in [6] it was shown that

$$
\tau_{p}(0, x) \leqq \exp \left[-\|x\|_{1} / \chi(p)\right] \quad \text { for model (i). }
$$

For model (ii) a similar exponential decay holds if $g$ has compact support, and for general exponentially decaying $g$ exponential decay of $\tau_{p}(x, y)$ (for $p<p_{c}$ ) can be shown using the same argument as that used for the Ising model in [7]. The exponential decay of the two-point function implies that $T, W_{a}$, and $H_{a_{1}, a_{2}}$ are finite for $p<p_{c}$.

Continuity of these quantities for $p<p_{c}$ then follows from the fact that $\tau_{p}(x, y)$ is monotonic and continuous in $p \in[0,1][5]$, together with the monotone convergence theorem. 
4.3. Proof of Proposition 4.3. In this section we restrict our attention to the nearest-neighbour model. We prove Proposition 4.3 and show that the infrared bound follows from $P_{4}$, thereby completing the proof of Theorem 1.1. We begin with a preliminary lemma in which bounds on $\bar{T}, \bar{W}$ and $\bar{H}$ are obtained from the assumption $P_{4}$.

4.3.1. Bounds on $\bar{T}, \bar{W}$, and $\bar{H}$. In this section we prove the following lemma.

Lemma 4.4. If $p<p_{c}$ and we assume $P_{4}$, then

$$
\begin{gathered}
\bar{T} \leqq 4 d^{-1}\left[K_{T}+\left(3 K_{T} / 8\right)^{1 / 2}\right] \equiv c_{1} d^{-1} \\
W_{1} \leqq d^{-1}\left[4 K_{W}+4\left(K_{W} K_{T} / 3\right)^{1 / 2}+\left(2 K_{T} / 3\right)^{1 / 2}\right] \equiv c_{2} d^{-1} \\
\bar{W} \leqq 4 K_{W}^{\prime} d^{-1}, \quad \bar{H} \leqq 40 K_{W} d^{-1} \\
W^{\prime} \leqq\left[16 K_{W}+16\left(K_{W} K_{T} / 3\right)^{1 / 2}+4\left(2 K_{W}\right)^{1 / 2}\right] d^{-1} \equiv c_{3} d^{-1} \\
\bar{W}^{\prime} \leqq 8 d^{-1}\left[4 K_{W}^{\prime}+4 K_{T} / 3+2\left(2 K_{T} / 3\right)^{1 / 2}+1\right] \leqq c_{4}\left[K_{W}^{\prime}+1\right] d^{-1}
\end{gathered}
$$

Here $W_{1}$ denotes $W_{a}$ where $a$ is any nearest neighbour of the origin. Note that the constants $c_{1}, c_{2}, c_{3}$ and $c_{4}$ depend only on $K_{T}$ and $K_{W}$, and not on $K_{W}^{\prime}$.

Proof. By Lemma 3.3, for any $a \in \mathbb{Z}^{d}$ we have

$$
T_{a} \leqq T+[3 T / 2 d]^{1 / 2},
$$

and the bound on $\bar{T}$ then follows if we assume $P_{4}$.

To obtain the inequality for $W_{1}$ we proceed as follows: Denoting a nearest neighbour of the origin by $e$,

$$
\begin{aligned}
& W_{1}=\sum_{x}|x|^{2} \tau(0, x) \tau(x, e) \\
& \leqq \sum_{x}|x| \tau(0, x)|x-e| \tau(e, x)+\sum_{x \neq e}|x| \tau(0, x)|e| \tau(e, x) \\
& +|e| \tau(0, e)|e| \tau(e, e) \text {. }
\end{aligned}
$$

Applying the Schwarz inequality to the first two terms on the right side, and using the fact that

gives

$$
\tau(0, e)^{2} \leqq(2 d)^{-1} \sum_{x \neq 0} \tau(0, x)^{2} \leqq(2 d)^{-1} T / 3
$$

$$
W_{1} \leqq W+\left[W \cdot \sum_{x \neq e} \tau(e, x)^{2}\right]^{1 / 2}+(T / 6 d)^{1 / 2} \leqq W+[W T / 3]^{1 / 2}+(T / 6 d)^{1 / 2} .
$$

The assumption $P_{4}$ then gives the bound $W_{1} \leqq c_{2} d^{-1}$.

We next give the proof that $P_{4}$ implies the bound $\bar{W} \leqq 4 K_{W}^{\prime} d^{-1}$. Although $P_{4}$ only involves $W_{a}$ for $\|a\|_{1} \leqq 2 \chi(p)\{(d+2) \ln [5 \chi(p)]+2 \ln d\}$, it is possible to estimate $\bar{W}=\sup _{a} W_{a}$ by using the exponential decay of the two-point function to bound $W_{a}$ for large values of $\|a\|_{1}$. In fact, we shall show that

$$
W_{a} \leqq d^{-1} \quad \text { for } \quad\|a\|_{1} \geqq 2 \chi(p)\{(d+2) \ln [5 \chi(p)]+2 \ln d\} .
$$


Given (4.2), it follows that

$$
\bar{W} \leqq \max \left\{4 K_{W} d^{-1}, 4 K_{W}^{\prime} d^{-1}, d^{-1}\right\}=4 K_{W}^{\prime} d^{-1} .
$$

To prove (4.2) we use the fact [6] that $\tau(0, x) \leqq \exp \left[-\|x\|_{1} / \chi(p)\right]$ to obtain

$$
\begin{aligned}
W_{a} & \leqq \sum_{x}|x|^{2} \exp \left[-\left(\|x\|_{1}+\|x-a\|_{1}\right) / \chi(p)\right] \\
& \leqq \sum_{x}|x|^{2} \exp \left[-\|x\|_{1} / 2 \chi(p)\right] \exp \left[-\|a\|_{1} / 2 \chi(p)\right] \\
& \leqq d[5 \chi(p)]^{d+2} \exp \left[-\|a\|_{1} / 2 \chi(p)\right] .
\end{aligned}
$$

The inequality for $\bar{H}$ is proved similarly, and we omit the proof. The bounds on $W^{\prime}$ and $\bar{W}^{\prime}$ follow from $P_{4}$ and Lemma 3.3.

4.3.2. Bounds on $\hat{\tau}(k)$. In Proposition 2.3 an expansion was given for the twopoint function $\tau(0, x)$. Since $\tau(0, x)$ decays exponentially for $p<p_{c}$, the Fourier transform $\hat{\tau}(k)$ exists. Taking the Fourier transform of both sides of the expansion and solving for $\hat{\tau}(k)$ yields

$$
\hat{\tau}(k)=\frac{\hat{G}^{(N)}(k)}{1-2 d p D(k)-\hat{\Pi}^{(N)}(k)},
$$

for any $p \in\left[0, p_{c}\right)$ and for any nonnegative integer $N$, where

$$
\hat{G}^{(N)}(k)=1+\sum_{n=0}^{N}(-1)^{n} \hat{g}_{n}(k)+(-1)^{N+1} \hat{R}_{N}(k),
$$

and

$$
\hat{\Pi}^{(N)}(k)=\sum_{n=0}^{N}(-1)^{n} \hat{\Pi}_{n}(k) .
$$

In this section we prove the following lemma.

Lemma 4.5. Fix $p \in\left[1 / 2 d, p_{c}\right)$ and $N \geqq 0$, and assume $P_{4}$. There exists a $d_{0}>6$, independent of $p$, such that for $d \geqq d_{0}$,

$$
\left|\hat{\Pi}^{(N)}(k)\right| \leqq c d^{-1}, \quad\left|\partial_{\mu}^{s} \hat{\Pi}^{(N)}(k)\right| \leqq c^{\prime} d^{-2}, \quad s=1,2 .
$$

If in addition $N$ is sufficiently large (depending on $d$ and $p$ ), then

$$
\begin{aligned}
& \hat{F}(k) \equiv 1-2 d p D(k)-\hat{\Pi}^{(N)}(k) \geqq\left(1-c^{\prime \prime} d^{-1}\right)(1-D(k)), \\
& \left|\hat{G}^{(N)}(k)-1\right| \leqq c d^{-1}, \quad\left|\partial_{\mu}^{s} \hat{G}^{(N)}(k)\right| \leqq c^{\prime} d^{-2}, \quad s=1,2,
\end{aligned}
$$

and

$$
0 \leqq \hat{\tau}(k) \leqq\left(1+c^{\prime \prime \prime} d^{-1}\right)(1-D(k))^{-1} .
$$

The constants $c, c^{\prime}, c^{\prime \prime}$ and $c^{\prime \prime \prime}$ depend only on $K_{T}$ and $K_{W}$ (and not on $K_{W}^{\prime}, p$, or $d$ ). Remark. For $p \in[0,1 / 2 d], \hat{\tau}(k) \leqq \hat{\tau}(0)=\chi(p) \leqq \chi(1 / 2 d)<\infty$. (It is known that $\hat{\tau}(k)$ is nonnegative [6].) Together with (4.7) this shows that for large $d$ the infrared bound stated in Theorem 1.1 is a consequence of $P_{4}$. 
Proof of Lemma 4.5. The bounds (4.4)-(4.6) are simply a combination of the results in Proposition 2.6, Lemma 3.2, and Lemma 4.4 with the assumption $P_{4}$. In the course of the proof, we use $c$ and $c^{\prime}$ to denote universal constants which may depend on $K_{T}$ and $K_{W}$, but not on $K_{W}^{\prime}{ }^{\prime} p$, or $d$. These constants may take on different values in different occurrences. First, by Lemma 3.2, Lemma 4.4 and $P_{4}$,

and

$$
0 \leqq \sum_{x} h_{n}(0, x) \leqq \begin{cases}c d^{-1} & n=0,1 \\ \left(c^{\prime} d^{-1}\right)^{n} & n \geqq 2\end{cases}
$$

$$
0 \leqq \sum_{x}|x|^{2} h_{n}(0, x) \leqq\left\{\begin{array}{ll}
c d^{-1} & n=0,1 \\
n^{2}\left(c^{\prime} d^{-1}\right)^{n-1} & n \geqq 2
\end{array} .\right.
$$

Now using Proposition 2.6 and (4.8-9) we immediately obtain

$$
\left|\hat{\Pi}^{(N)}(k)\right| \leqq 2 d p \sum_{n=0}^{N} \sum_{x} h_{n}(0, x) \leqq c d^{-1}
$$

The bound on $\partial_{\mu}^{s} \hat{\Pi}^{(N)}(k)$ is obtained similarly.

To bound $\left|\hat{G}^{(N)}(k)-1\right|$ we use Proposition 2.6 to obtain

$$
\left|\hat{G}^{(N)}(k)-1\right| \leqq \sum_{n=0}^{N} \sum_{x} h_{n}(0, x)+2 d p \chi(p) \sum_{x} h_{N}(0, x),
$$

and then note that the first term on the right side is bounded above by $c d^{-1}$, while the second is bounded above by $2 d p \chi(p)\left(c^{\prime} d^{-1}\right)^{N}$. For $N$ sufficiently large, this last quantity is bounded above by $c d^{-1}$, and the desired bound on $\left|\hat{G}^{(N)}(k)-1\right|$ follows.

The bounds on $\left|\partial_{\mu}^{s} \hat{G}^{(N)}(k)\right|$ are obtained in a similar fashion. To estimate $\left|\partial_{\mu}^{s} \hat{R}_{N}(k)\right|$ we use the bound from Proposition 2.6 and the inequality

$$
\sum_{x}|x|^{2} \tau(0, x) \leqq \sum_{x}|x|^{2} \exp \left[-\|x\|_{1} / \chi(p)\right] \leqq d(3 \chi(p))^{d+2}
$$

To obtain (4.5) we first observe that by (4.6),

$$
1-2 d p-\hat{\Pi}^{(N)}(0)=\hat{G}^{(N)}(0) \chi(p)^{-1} \geqq 0 .
$$

Therefore $2 d p \leqq 1+c d^{-1}$, and also

$$
\begin{aligned}
1-2 d p D(k)-\hat{\Pi}^{(N)}(k)= & 1-2 d p D(k)-\hat{\Pi}^{(N)}(k)-\left[1-2 d p-\hat{\Pi}^{(N)}(0)\right] \\
& +\left[1-2 d p-\hat{\Pi}^{(N)}(0)\right] \\
\geqq & 2 d p[1-D(k)]+\hat{\Pi}^{(N)}(0)-\hat{\Pi}^{(N)}(k) .
\end{aligned}
$$

By Proposition 2.6 and (2.24),

$$
\begin{aligned}
\hat{\Pi}^{(N)}(0)-\hat{\Pi}^{(N)}(k) & \geqq-\sum_{\substack{n=1 \\
n: \text { odd }}}^{N}\left[\hat{\Pi}_{n}(0)-\hat{\Pi}_{n}(k)\right] \\
& \geqq \frac{\pi^{2}}{4}(1-D(k)) \cdot 2 d p\left[-\sum_{\substack{n=1 \\
n: \text { odd }}}^{N} \sum_{x}\left(|x|^{2}+1\right) h_{n}(0, x)\right] .
\end{aligned}
$$

Now, using (4.8-9) gives (4.5).

Finally the infrared bound (4.7) follows from (4.3) and (4.5-6). 
4.3.3. Proof that $P_{4} \Rightarrow P_{3}$. In this section we use the consequences of $P_{4}$ given in Lemma 4.5 to derive $P_{3}$. This completes the proof of Proposition 4.3 and thus of Theorem 1.1. We fix an $N$ large enough (depending on $p$ ) that (4.5-7) hold, and omit the superscript $(N)$ to simplify the notation. As usual we use $c, c^{\prime}$ and $c^{\prime \prime}$ to denote universal constants which depend only on $K_{T}$ and $K_{W}$, but not on $K_{W}^{\prime}, p$, or $d$. These constants may represent different values in different occurrences. Throughout this section we fix $p \in\left[1 / 2 d, p_{c}\right)$.

The statement $P_{3}$ consists of five inequalities (given in Proposition 4.3). We obtain these one by one, beginning with $2 d p \leqq 3$

(a) $2 d p \leqq 3$ : In the proof of Lemma 4.5 it was shown that for $p \in\left[1 / 2 d, p_{c}\right.$ ),

and hence by (4.4)

$$
1-2 d p-\hat{\Pi}(0) \geqq 0,
$$

$$
2 d p \leqq 1-\hat{\Pi}(0) \leqq 1+c d^{-1} .
$$

The right side of (4.10) is less than 3 for $d \geqq c / 2$.

(b) The bound on $T$ : By definition

$$
\begin{aligned}
T & =(2 \pi)^{-d} \int d^{d} k\left[\hat{\tau}(k)^{3}-\tau(0,0)^{3}\right] \\
& =(2 \pi)^{-d} \int d^{d} k\left[(2+\hat{\tau}(k))(\hat{\tau}(k)-1)^{2}-(2+\tau(0,0))(\tau(0,0)-1)^{2}\right],
\end{aligned}
$$

and $T_{G}$ is obtained by replacing $\hat{\tau}(k)$ by $[1-D(k)]^{-1}$. (In fact, for percolation $\tau(0,0)=1$ and the second term on the right side of (4.11) is zero.) By (4.7), for $d \geqq 4 c^{\prime \prime \prime}$,

$$
0 \leqq 2+\hat{\tau}(k) \leqq 2+\left(1+c^{\prime \prime \prime} d^{-1}\right)[1-D(k)]^{-1} \leqq 2+\frac{5}{4}[1-D(k)]^{-1} .
$$

Also,

$$
\hat{\tau}(k)-1=\frac{2 d p D(k)+\hat{G}(k)-1+\hat{\Pi}(k)}{1-2 d p D(k)-\hat{\Pi}(k)} .
$$

We define

$$
\hat{F}(k)=1-2 d p D(k)-\hat{\Pi}(k) .
$$

Then by (4.4-6),

$$
\begin{aligned}
{[\hat{\tau}(k)-1]^{2} } & \leqq \hat{F}(k)^{-2} 2\left\{(2 d p D(k))^{2}+(|\hat{G}(k)-1|+|\hat{\Pi}(k)|)^{2}\right\} \\
& \leqq 20[1-D(k)]^{-2}\left(D(k)^{2}+c^{\prime} d^{-2}\right),
\end{aligned}
$$

for $d$ sufficiently large. Using the fact that the integral of $[1-D(k)]^{-m}$ is bounded uniformly in $d \geqq 7$ for $m=1,2,3$ (see Appendix B of [20] or Eq. (3.12) of [31] for a proof), it follows from (4.11), (4.12) and (4.14) that

$$
\begin{aligned}
T & \leqq 20(2 \pi)^{-d} \int d^{d} k\left(2+\frac{5}{4}[1-D(k)]^{-1}\right) D(k)^{2}[1-D(k)]^{-2}+c^{\prime \prime} d^{-2} \\
& \leqq 25 T_{G}+c^{\prime \prime} d^{-2} \leqq K_{T} d^{-1}+c^{\prime \prime} d^{-2} \leqq 3 K_{T} d^{-1},
\end{aligned}
$$


for $d$ sufficiently large, where we used the fact that $C(0,0)-1 \leqq c d^{-1}$ (which follows from Appendix B of [20] or Lemma 3.1 of [31]) together with the form of $T_{G}$ described below (4.11).

(c) The bound on $W$ : By definition and the Parseval relation,

$$
W=\sum_{x}[|x| \tau(0, x)]^{2}=\sum_{\mu=1}^{d}(2 \pi)^{-d} \int d^{d} k\left[\partial_{\mu} \hat{\tau}(k)\right]^{2} .
$$

Differentiation of (4.3) gives

$$
\begin{aligned}
\partial_{\mu} \hat{\tau}(k)= & \hat{F}(k)^{-2} \hat{G}(k) 2 d p \partial_{\mu} D(k)+\hat{F}(k)^{-1} \partial_{\mu} \hat{G}(k) \\
& +\hat{F}(k)^{-2} \hat{G}(k) \partial_{\mu} \hat{\Pi}(k) .
\end{aligned}
$$

By (4.15-16)

$$
\begin{aligned}
W \leqq & \sum_{\mu=1}^{d} 3(2 \pi)^{-d} \int d^{d} k\left\{\hat{F}(k)^{-4} 9\left[\hat{G}(k) \partial_{\mu} D(k)\right]^{2}+\hat{F}(k)^{-2}\left(\partial_{\mu} \hat{G}(k)\right)^{2}\right. \\
& \left.+\hat{F}(k)^{-4}\left(\hat{G}(k) \partial_{\mu} \hat{\Pi}(k)\right)^{2}\right\}
\end{aligned}
$$

We use (4.5) to estimate the powers of $\hat{F}(k)$ occurring in the three terms on the right-hand side. By (4.6), the first term (including the sum over $\mu$ ) is bounded by $30 W_{G}$ for $d$ sufficiently large, and by (4.6) the second is bounded above by $c d^{-3}$. To bound the third term, we first note that by symmetry $\partial_{\mu} \hat{\Pi}(k)$ is equal to zero for any $k$ for which $k_{\mu}=0$. Denoting by $\tilde{k}$ the result of replacing the $\mu^{\text {th }}$ component of $k$ by zero, it follows from Taylor's theorem that

$$
\partial_{\mu} \hat{\Pi}(k)=\partial_{\mu} \hat{\Pi}(k)-\partial_{\mu} \hat{\Pi}(\tilde{k})=k_{\mu} \partial_{\mu}^{2} \hat{\Pi}\left(k^{*}\right),
$$

where $k^{*}$ is a point on the line segment joining $k$ and $\tilde{k}$. By (4.4) and (4.6), the third term in (4.17) is bounded above by

$$
c d^{-4}(2 \pi)^{-d} \int d^{d} k[1-D(k)]^{-4} k^{2} .
$$

Since $k^{2} d^{-1} \leqq \frac{\pi^{2}}{2}[1-D(k)]$, this is bounded above by $c^{\prime} d^{-3}$. This gives the desired bound on $W$, for $d$ sufficiently large.

(d) The bound on $W_{a}$ : For $|a|=1$, it was shown in Lemma 4.4 that $W_{a} \leqq c_{2} d^{-1}$ and hence $W_{a} \leqq 3 K_{W}^{\prime} d^{-1}$ if we take $K_{W}^{\prime} \geqq c_{2} / 3$.

For the case $|a|>1$ we again use Fourier transforms and write

$$
W_{a}=\sum_{x}|x|^{2} \tau(0, x) \tau(x, a)=-(2 \pi)^{-d} \int d^{d} k \hat{\tau}(k) \sum_{\mu=1}^{d} \hat{\tau}_{\mu \mu}(k) e^{i k \cdot a},
$$

where we have used subscripts to denote partial derivatives with respect to $k_{\mu}$. Now

$$
\hat{\tau}_{\mu \mu}(k)=\hat{F}^{-1} \hat{G}_{\mu \mu}-2 \hat{F}^{-2} \hat{G}_{\mu} \hat{F}_{\mu}-\hat{F}^{-2} \hat{G} \hat{F}_{\mu \mu}+2 \hat{F}^{-3} \hat{G} \hat{F}_{\mu}^{2},
$$

with

$$
\hat{F}_{\mu}=-2 d p D_{\mu}-\hat{\Pi}_{\mu}=2 p \sin k_{\mu}-\hat{\Pi}_{\mu}
$$


and

$$
\hat{F}_{\mu \mu}=-2 d p D_{\mu \mu}-\hat{\Pi}_{\mu \mu}=2 p \cos k_{\mu}-\hat{\Pi}_{\mu \mu}
$$

Therefore

$$
\begin{aligned}
W_{a}= & -\sum_{\mu=1}^{d}(2 \pi)^{-d} \int d^{d} k e^{i k \cdot a} \\
& \cdot\left[\hat{\tau}^{2} \hat{G}^{-1} \hat{G}_{\mu \mu}-2 \hat{\tau}^{3} \hat{G}^{-2} \hat{G}_{\mu} \hat{F}_{\mu}-\hat{\tau}^{3} \hat{G}^{-1} \hat{F}_{\mu \mu}+2 \hat{\tau}^{4} \hat{G}^{-2} \hat{F}_{\mu}^{2}\right] .
\end{aligned}
$$

We estimate the terms on the right side using (4.4), (4.6) and (4.7), together with the fact that the integral of $[1-D(k)]^{-m}$ is uniformly bounded for $m \leqq 3$ and $d \geqq 7$. The first term (including the sum over $\mu$ ) is less than $c d^{-1}$, and since $\hat{\bar{F}}_{\mu} \leqq c^{\prime} d^{-1}$ the second term is less than $c d^{-2}$. The estimate on the third term is more involved.

The contribution to $W_{a}$ arising from the gaussian part $2 p \cos k_{\mu}$ of $\hat{F}_{\mu \mu}$ in the third term on the right side of (4.20) is

$$
A_{a} \equiv 2 d p(2 \pi)^{-d} \int d^{d} k \hat{\tau}(k)^{3}\left[1+\hat{G}(k)^{-1}-1\right] d^{-1} \sum_{\mu=1}^{d} \cos k_{\mu} e^{i k \cdot a} .
$$

To estimate the contribution $A_{a}^{\prime}$ to $A_{a}$ due to the 1 in the square brackets we write

to get

$$
d^{-1} \sum_{\mu=1}^{d} \cos k_{\mu}=(2 d)^{-1} \sum_{v,|v|=1} e^{i k \cdot v}
$$

$$
\begin{aligned}
\left|A_{a}^{\prime}\right| & \leqq 2 d p(2 d)^{-1} \sum_{v,|v|=1}\left|(2 \pi)^{-d} \int d^{d} k \hat{\tau}(k)^{3} e^{i k \cdot(a+v)}\right| \\
& =2 d p(2 d)^{-1} \sum_{v,|v|=1} T_{a+v} \leqq 3 \bar{T} \leqq 3 c_{1} d^{-1} .
\end{aligned}
$$

(Note that $a+v \neq 0$ here since $|a| \neq 1$.) Then by (4.6-7) and the fact that $2 d p \leqq 3$ we have

$$
\left|A_{a}\right| \leqq\left|A_{a}^{\prime}\right|+\left|A_{a}-A_{a}^{\prime}\right| \leqq 3 c_{1} d^{-1}+c d^{-1} \leqq c^{\prime} d^{-1} .
$$

Using (4.4) and (4.6-7) it is not difficult to see that the contribution to $W_{a}$ due to the $\hat{\Pi}_{\mu \mu}$ term in $\hat{F}_{\mu \mu}$ in the third term on the right side of (4.20) is also bounded by $c d^{-1}$.

Finally the last term on the right hand side of (4.20) can be bounded above by $c d^{-1}$ using an argument involving Taylor's Theorem which is similar to that used in the last paragraph of (c). (Note that it is necessary to exploit the fact that $F_{\mu}(0)=0$ if we are to avoid quantities which diverge in more than six dimensions.) All the constants $c$ and $c^{\prime}$ encountered here depend only on $K_{T}$ and $K_{W}$, so it is possible to choose $K_{W}^{\prime}$ (depending on $K_{T}$ and $K_{W}$ ) sufficiently large that the desired bound $W_{a} \leqq 3 K_{W}^{\prime} d^{-1}$ holds, and that the corresponding gaussian quantity is less than $K_{W}^{\prime} d^{-1}$ (as claimed in Sect. 4.1). 
(e) The bound on $H$ : Using the Fourier transform, and then integrating by parts, we can rewrite Eq. (3.3) as

$$
\begin{aligned}
H_{a_{1}, a_{2}}= & \int_{j=1}^{3} \frac{d^{d} k_{j}}{(2 \pi)^{d}} e^{i\left(k_{1} \cdot a_{1}-k_{2} \cdot a_{2}\right)} \hat{\tau}\left(k_{1}\right)^{2} \hat{\tau}\left(k_{2}\right)^{2} \hat{\tau}\left(k_{1}-k_{2}\right) \hat{\tau}\left(k_{1}-k_{3}\right) \\
& \cdot \hat{\tau}\left(k_{3}-k_{2}\right)\left[-\sum_{\mu=1}^{d} \partial_{\mu}^{2} \hat{\tau}\left(k_{3}\right)\right] \\
= & \sum_{\mu=1}^{d} \int \prod_{j=1}^{3} \frac{d^{d} k_{j}}{(2 \pi)^{d}} e^{i\left(k_{1} \cdot a_{1}-k_{2} \cdot a_{2}\right)} \hat{\tau}\left(k_{1}\right)^{2} \hat{\tau}\left(k_{2}\right)^{2} \hat{\tau}\left(k_{1}-k_{2}\right) \\
& \cdot\left\{\hat{\tau}\left(k_{1}-k_{3}\right)\left(\partial_{\mu} \hat{\tau}\left(k_{3}\right)\right)\left(\partial_{\mu} \hat{\tau}\left(k_{3}-k_{2}\right)\right)-\hat{\tau}\left(k_{3}-k_{2}\right)\left(\partial_{\mu} \hat{\tau}\left(k_{3}\right)\right)\left(\partial_{\mu} \hat{\tau}\left(k_{1}-k_{3}\right)\right)\right\} .
\end{aligned}
$$

Taking absolute values of the integrand on the right side and using the symmetry between $k_{1}$ and $k_{2}$ leads to

$$
\begin{aligned}
H_{a_{1}, a_{2}} \leqq & \sum_{\mu=1}^{d} \int_{j=1}^{3} \frac{d^{d} k_{j}}{(2 \pi)^{d}} \hat{\tau}\left(k_{1}\right)^{2} \hat{\tau}\left(k_{2}\right)^{2} \hat{\tau}\left(k_{1}-k_{2}\right) \\
& \cdot \hat{\tau}\left(k_{1}-k_{3}\right)\left\{\left(\partial_{\mu} \hat{\tau}\left(k_{3}\right)\right)^{2}+\left(\partial_{\mu} \hat{\tau}\left(k_{3}-k_{2}\right)\right)^{2}\right\} .
\end{aligned}
$$

Rewriting this upper bound in $x$-space gives

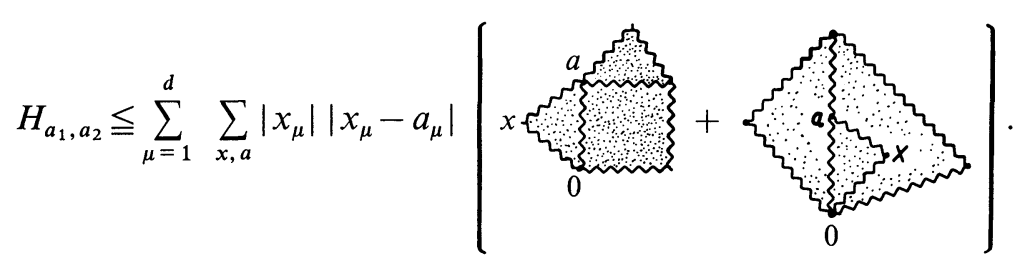

Now we use the basic inequality (3.5) to bound the right side. First,

$$
\begin{aligned}
& \left.\sum_{\mu=1}^{d} \sum_{x, a}\left|x_{\mu}\right|\left|x_{\mu}-a_{\mu}\right| x\right) \\
& \leqq\left[\sup _{a} \sum_{\mu=1}^{d} \sum_{x}\left|x_{\mu}\right|\left|x_{\mu}-a_{\mu}\right| x \text { mิm }_{\text {man }}^{a}\right] \\
& \leqq W\left[\sup _{a}\left\{\begin{array}{ll}
0 & a \\
& \xi
\end{array}\right](1+T) \leqq W(1+T)(1+\bar{T}),\right.
\end{aligned}
$$


where in the second inequality the sum over $x$ was estimated using the Schwarz inequality. The second term on the right side of (4.21) can be bounded similarly:

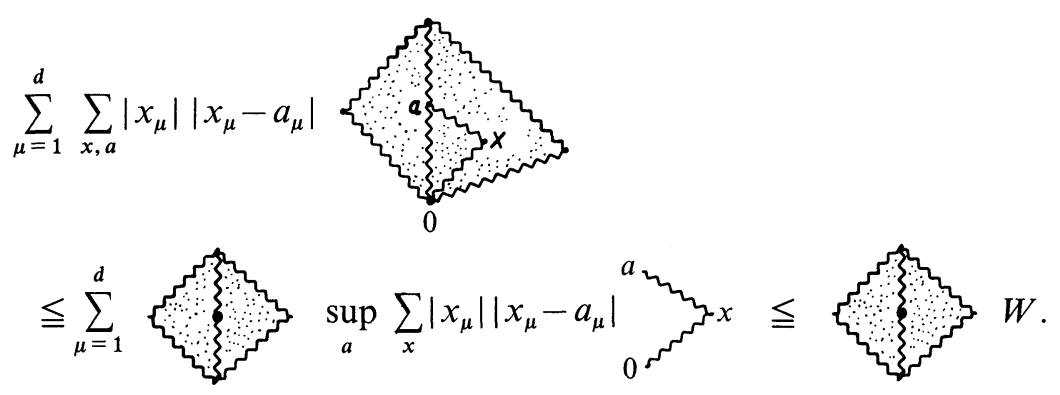

Now we use (4.7) to bound the diagram on the right side of (4.23) in terms of its gaussian value:

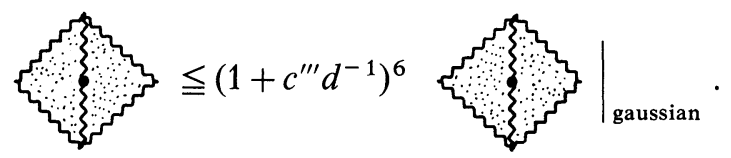

The gaussian diagram on the right side is finite for $d>6$ and we have verified numerically that it is bounded by 3 uniformly in $d \geqq 9$.

For $d$ sufficiently large, depending on $K_{T}$ and $K_{W}$, it follows from (4.21-24) that

$$
H_{a_{1}, a_{2}} \leqq \frac{3}{2}\left(1+\left.\sum_{\text {हैa }}^{3}\right|_{\text {gaussian }}\right) W \leqq 30 K_{W} d^{-1} .
$$

This is the desired bound. Also, this calculation shows that the gaussian counterpart of $H_{a_{1}, a_{2}}$ is bounded above by $10 K_{W} d^{-1}$ for $d$ sufficiently large, as claimed in Sect. 4.1.

\subsection{Proof that $v_{2}=1 / 2$}

By definition [Eq. (1.6)],

$$
\xi_{2}(p)^{2}=-\sum_{\mu=1}^{d} \frac{\hat{\tau}_{\mu \mu}(0)}{\hat{\tau}(0)}
$$

Since $\hat{F}^{-1}=\hat{G}^{-1} \hat{\tau}, \hat{F}_{\mu}(0)=0$, and $\hat{\tau}(0)=\chi(p)$, it follows from (4.19) and (4.26) that

$$
\begin{aligned}
\xi_{2}(p)^{2} & =-\sum_{\mu=1}^{d}\left[\hat{G}(0)^{-1} \hat{G}_{\mu \mu}(0)-\chi(p) \hat{G}(0)^{-1} \hat{F}_{\mu \mu}(0)\right] \\
& =-\sum_{\mu=1}^{d} \hat{G}(0)^{-1}\left[\hat{G}_{\mu \mu}(0)-\chi(p)\left(2 p-\hat{\Pi}_{\mu \mu}(0)\right)\right] .
\end{aligned}
$$


By Lemma 4.5 (since we have proved $P_{3}, P_{4}$ does hold), it follows that for $1 / 2 d \leqq p<p_{c}$,

$$
\xi_{2}(p)^{2}=O\left(d^{-1}\right)+\left[1+O\left(d^{-1}\right)\right] \chi(p),
$$

and hence for some constant $C>0$,

$$
C^{-1} \chi(p) \leqq \xi_{2}(p)^{2} \leqq C \chi(p) \text { for } p \in\left[1 / 2 d, p_{c}\right)
$$

Since $\chi(p) \sim\left(p_{c}-p\right)^{-1}$ follows from the triangle condition, $\xi_{2}(p) \sim\left(p_{c}-p\right)^{-1 / 2}$, i.e., $v_{2}=1 / 2$.

\section{Proof of the Main Results (Theorem 1.2) for the Spread-out Models}

In this section we prove Theorem 1.2 by adapting the proof of Theorem 1.1 to the spread-out models introduced in Sect.1.1. These models are defined by

$$
p_{0 x}=p \cdot L^{-d} g(x / L),
$$
where $g$ is a nonnegative function on $\mathbb{R}^{d}$ such that $\partial^{I} g\left(\partial^{I} g \equiv \prod_{\mu \in I} \partial_{\mu} g, I \subset\{1,2, \ldots, d\}\right)$
is piecewise continuous, which satisfies:

$$
\int g(x) d x=1, \quad g \cdot e^{\delta\|x\|_{1}} \in L_{\infty}\left(\mathbb{R}^{d}\right) \text { for some } \delta>0,
$$

$\int\left|\partial^{I} g(x)\right| d x<\infty$, where the derivative is interpreted as a distribution,

$g$ is invariant under rotations by $\pi / 2$ and reflections

in the coordinate hyperplanes.

To simplify the technicalities we have required that $g$ be exponentially decaying, although some weaker decay can also be handled. The fact that Theorem 1.2 holds independently of the exact form of $g$ is an illustration of universality.

Just as for the nearest-neighbour model, $p_{c}=\sup \{p \mid \chi(p)<\infty\} \in(0,1)$. The only significant difference between the analysis of the model (ii) and the nearestneighbour model is due to the change in gaussian propagator. (The situation is similar for the long-range weakly self-avoiding walk studied in [40], although in that work the $1 / r^{2}$ decay considered was sufficiently slow to change the upper critical dimension, unlike the models under study here.)

The hypotheses on $g$ are used indirectly, via certain of their consequences, to prove Theorem 1.2. To state these consequences we introduce the following notation. We consider simple random walks with transition probabilities

$$
p_{0 x}^{(L)}=p_{L} \cdot L^{-d} g(x / L) .
$$

Here $p_{L}$ is defined so that $D_{L}(0)=1$, where

$$
D_{L}(k) \equiv p_{L} \sum_{x} L^{-d} g(x / L) e^{i k \cdot x}
$$

The gaussian propagator (in $k$-space) for the model is then

$$
\hat{C}_{L}(k)=\left[1-D_{L}(k)\right]^{-1}
$$


and we define $T_{L}$ and $W_{L}$ the same way that $T_{G}$ and $W_{G}$ were defined (under Proposition 4.3), using this propagator. We also define

$$
S=\sum_{x}|x|^{2} p_{0 x}^{(L)}
$$

(As will be seen in Sect. 5.2, $S=O\left(L^{2}\right)$; clearly $S \geqq 1$.)

To prove Theorem 1.2 we will use the following lemma.

Lemma 5.1. The hypotheses (5.1) on $g$ imply that given $d>6$ and $\varepsilon>0$, there is an $L_{0}$, depending on $\varepsilon$ and $g$, such that for $L \geqq L_{0}$ the following conditions are satisfied:

$$
\begin{aligned}
& \sup _{x} p_{0 x}^{(L)}, \quad \sum_{x}\left(p_{0 x}^{(L)}\right)^{2} \leqq \varepsilon / S, \\
& \sup p_{0 x}^{(L)}|x|^{2} \leqq \varepsilon, \\
& \sum_{x}\left(p_{0 x}^{(L)}\right)^{2}|x|^{4} \leqq S \varepsilon, \\
& 1-D_{L}(k) \geqq|k|^{2} / 3 \pi^{2} d, \\
& C_{L}(0,0)-1 \leqq \varepsilon / S \text {, } \\
& T_{L} \leqq \varepsilon / S, \\
& W_{L} \leqq \varepsilon, \\
& (2 \pi)^{-d} \int d^{d} k \sum_{\mu=1}^{d}\left|\partial_{\mu}^{2} D_{L}(k)\right|\left[1-D_{L}(k)\right]^{-3} \leqq 40 \varepsilon, \\
& \text { हु }
\end{aligned}
$$

The values of the constants appearing in these conditions are not sharp and can be adjusted.

This section is organized as follows. In Sect. 5.1 Theorem 1.2 is proved, assuming Lemma 5.1. In Sect. 5.2 the proof of Lemma 5.1 is given.

\subsection{Proof of Theorem 1.2 Assuming Lemma 5.1}

In this section we prove that the triangle condition and the infrared bound follow if conditions (5.6-14) are satisfied, for $\varepsilon$ sufficiently small. Theorem 1.2 then follows from Lemma 5.1. The basic structure of the proof is exactly as in Theorem 1.1: the result follows from Lemmas 4.1 and 4.2 and the following analogue of Proposition 4.3, together with a proof that the infrared bound follows from the analogue of $P_{4}$. The proof that $v_{2}=1 / 2$ proceeds the same way as for the nearest-neighbour model in Sect. 4.4.

The analogue of Proposition 4.3 is the following.

Proposition 5.2. There is an $\varepsilon_{0}>0$ such that if an independent bond percolation model on $\mathbb{Z}^{d}(d>6)$ satisfies conditions $(5.6-14)$ for some $\varepsilon \leqq \varepsilon_{0}$, then for any fixed 
$p \in\left[p_{L}, p_{c}\right), P_{4}$ implies $P_{3}$, where $P_{\alpha}$ is the statement that the following set of inequalities holds:

$$
\begin{array}{r}
T \leqq \alpha \cdot 25 \varepsilon / S, \quad W \leqq \alpha \cdot 30 \varepsilon, \quad \frac{p}{p_{L}} \leqq \alpha \\
W_{a} \leqq \alpha K^{\prime} \varepsilon \text { for }\|a\|_{1} \leqq M_{1}(p), \\
H_{a_{1}, a_{2}} \leqq \alpha \cdot 500 \varepsilon \text { for } \max _{i=1,2}\left\|a_{i}\right\|_{1} \leqq M_{2}(p) .
\end{array}
$$

The universal constant $K^{\prime}$ is determined in the proof of the proposition. The quantities $M_{i}(p)$ are finite for $p<p_{c}$ and are defined such that

$$
W_{a} \leqq \varepsilon \text { for }\|a\|_{1} \geqq M_{1}(p), \quad H_{a_{1}, a_{2}} \leqq \varepsilon \quad \text { for } \quad \max _{i=1,2}\left\|a_{i}\right\|_{1} \geqq M_{2}(p) .
$$

The existence of such constants follows from the exponential decay of $\tau_{p}(x, y)$ (discussed in the proof of Lemma 4.2), as for the nearest-neighbour model.

Proof of Proposition 5.2. The remainder of this section is devoted to the proof of Proposition 5.2. In the course of the proof it will be shown that the infrared bound is a consequence of $P_{4}$. The first step is the following lemma.

Lemma 5.3. If $p<p_{c}$ and we assume $P_{4}$, with $\varepsilon$ sufficiently small, then there are constants $c_{i}$, independent of $p$ and $K^{\prime}$, such that

$$
\bar{T} \leqq c_{1} \varepsilon^{1 / 2}, \quad \bar{W} \leqq 4 K^{\prime} \varepsilon, \quad \bar{H} \leqq 2000 \varepsilon, \quad W^{\prime} \leqq c_{2} \varepsilon, \quad W_{a}^{\prime} \leqq c_{3} K^{\prime} \varepsilon .
$$

Proof. The bounds on $\bar{W}$ and $\bar{H}$ follow immediately from $P_{4}$ and the definition of $M_{i}(p)$. The bound on $\bar{T}$ follows from $P_{4}$ and the fact that by Lemma 3.3,

$$
T_{a} \leqq T+(3 T / 2 d)^{1 / 2} \text {. }
$$

The bounds on $W^{\prime}$ and $W_{a}^{\prime}$ also follow directly from Lemma 3.3, conditions (5.614) and $P_{4}$. To bound $\sum_{v} p_{0 v}|v|$ we use the Schwarz inequality:

$$
\sum_{v} p_{0 v}|v| \leqq\left[\sum_{x} p_{0 x} \cdot \sum_{y} p_{0 y}|y|^{2}\right]^{1 / 2}=\frac{p}{p_{L}} S^{1 / 2} .
$$

In conditions (5.6-14) the powers of $S$-are chosen in such a way as to cancel the powers of $S$ which arise from the bounds on $W_{1}^{\prime}$ and $W_{a}^{\prime}$ of Lemma 3.3.

Lemma 5.3 combined with Lemma 3.2 yields the following bounds, in which $c$ and $c^{\prime}$ are constants independent of $p$ and $K^{\prime}$, and $\varepsilon$ is taken sufficiently small depending on $K^{\prime}$,

$$
\begin{array}{r}
0 \leqq \sum_{x} h_{n}(0, x) \leqq\left\{\begin{array}{ll}
c \varepsilon / S & \text { for } n=0,1 \\
\frac{\varepsilon}{S}\left(c^{\prime} \varepsilon\right)^{(n-1) / 2} & \text { for } n \geqq 2
\end{array},\right. \\
0 \leqq \sum_{x}|x|^{2} h_{n}(0, x) \leqq\left\{\begin{array}{ll}
c \varepsilon & \text { for } n=0,1 \\
c \varepsilon n^{2}\left(c^{\prime} \varepsilon\right)^{(n-2) / 2} & \text { for } n \geqq 2
\end{array} .\right.
\end{array}
$$

These bounds can be used in conjunction with Proposition 2.6 to control $\hat{\tau}(k)$, just as in Sect. 4.3.2. 
Indeed, using the notation under (4.3), (2.2) becomes

$$
\hat{\tau}(k)=\hat{G}^{(N)}(k) /\left[1-\frac{p}{p_{L}} D_{L}(k)-\hat{\Pi}^{(N)}(k)\right] .
$$

Proceeding exactly as in the proof of Lemma 4.5 the following inequalities, in which the constants $c, c^{\prime}$, and $c^{\prime \prime}$ are independent of $d$ and $K^{\prime}$, are obtained.

$$
\left|\hat{\Pi}^{(N)}(k)\right| \leqq c \varepsilon / S, \quad\left|\partial_{\mu_{i}}^{s} \hat{\Pi}^{(N)}(k)\right| \leqq c^{\prime} \varepsilon / d, \quad s=1,2 .
$$

If in addition $N$ is sufficiently large, depending on $p$, then for $p \in\left[p_{L}, p_{c}\right)$,

$$
\begin{gathered}
\hat{F}(k) \equiv 1-\frac{p}{p_{L}} D_{L}(k)-\hat{\Pi}^{(N)}(k) \geqq\left(1-c^{\prime \prime} \varepsilon\right)\left(1-D_{L}(k)\right), \\
\left|\hat{G}^{(N)}(k)-1\right| \leqq c \varepsilon / S, \quad\left|\partial_{\mu}^{s} \hat{G}^{(N)}(k)\right| \leqq c^{\prime} \varepsilon / d, \quad s=1,2,
\end{gathered}
$$

and

$$
\hat{\tau}(k) \leqq\left(1+c^{\prime \prime} \varepsilon\right)\left(1-D_{L}(k)\right)^{-1}
$$

The proof of (5.18) uses (5.9), and the fact that (5.19) holds for sufficiently large $N$ uses the exponential decay of $\tau_{p}(x, y)$.

We now turn to the proof that $P_{4}$ implies $P_{3}$, and proceed step by step through the five inequalities in the statement of $P_{4}$. Most of the analysis is identical to that of Sect. 4.3.3, using (5.17-20), and we refer the reader to that section. Now $\varepsilon$ plays the role of $1 / d$. To simplify the notation we omit the superscript $(N)$.

(a) $\frac{p}{p_{L}} \leqq 3$ : This is identical to the nearest neighbour case in Sect. 4.4.3(a).

(b) The bound on $T$ : This bound can also be obtained by the same argument as in the nearest neighbour case, by faithfully following Sect.4.3.3(b). Note that the integral of $\left(1-D_{L}\right)^{-m}(m=1,2,3)$ is bounded, in fact close to one, by conditions (5.10-11) and the Hölder inequality. In the last step (5.10) is used.

(c) The bound on $W$ : This case follows 4.3.3(c).

(d) The bound on $W_{a}$ : The treatment of this case is the only one which differs significantly from the nearest neighbour case. Direct calculation as in (4.20) gives

$$
\begin{aligned}
W_{a}= & -\sum_{\mu=1}^{d}(2 \pi)^{-d} \int d^{d} k e^{i k \cdot a} \\
& \cdot\left[\hat{\tau}^{2} \hat{G}^{-1} \hat{G}_{\mu \mu}-2 \hat{\tau}^{3} \hat{G}^{-2} \hat{G}_{\mu} \hat{F}_{\mu}-\hat{\tau}^{3} \hat{G}^{-1} \hat{F}_{\mu \mu}+2 \hat{\tau}^{4} \hat{G}^{-2} \hat{F}_{\mu}^{2}\right],
\end{aligned}
$$

with

$$
\hat{F}_{\mu}(k)=-\frac{p}{p_{L}} \partial_{\mu} D_{L}(k)-\partial_{\mu} \hat{\Pi}(k)
$$

and

$$
\hat{F}_{\mu \mu}(k)=-\frac{p}{p_{L}} \partial_{\mu}^{2} D_{L}(k)-\partial_{\mu}^{2} \hat{\Pi}(k) .
$$

In (5.21) the first term and the contributions to the second and third terms from the derivatives of $\hat{\Pi}$ can be bounded just as in Sect. 4.3.3(d), using (5.17-20). Their sum is bounded by $O(\varepsilon)$. 
The fourth term is treated as follows. First we use

$$
\hat{F}_{\mu}^{2} \leqq 2\left(\frac{p}{p_{L}} \partial_{\mu} D_{L}\right)^{2}+2\left(\partial_{\mu} \hat{\Pi}\right)^{2},
$$

and for the second term use the mean value theorem and (5.17) to bound $\left|\partial_{\mu} \hat{\Pi}\right|$ by $O(\varepsilon)\left|k_{\mu}\right|$, and then argue as in Sect. 4.3.3(d) to bound this contribution to (5.21) by $O(\varepsilon)^{2}$. The contribution from the first term is bounded by

$$
\text { const } \sum_{\mu=1}^{d}(2 \pi)^{-d} \int d^{d} k\left(\partial_{\mu} D_{L}\right)^{2}\left(1-D_{L}\right)^{-4}=\text { const } W_{L} \leqq \text { const } \varepsilon,
$$

using (5.12).

Now we are left with two terms: the contributions from derivatives of $D_{L}$ in the second and third terms of (5.21). We first consider the second term and use (5.19-20) and the Schwarz inequality to obtain

$$
\begin{aligned}
& \left|\sum_{\mu=1}^{d}(2 \pi)^{-d} \int d^{d} k 2 \hat{\tau}^{3} \hat{G}^{-2} \hat{G}_{\mu} \partial_{\mu} D_{L}\right| \leqq \text { const } \sum_{\mu=1}^{d} \int d^{d} k\left|\hat{G}_{\mu} \partial_{\mu} D_{L}\right|\left(1-D_{L}\right)^{-3} \\
& \quad \leqq \text { const }\left[\sum_{\mu} \int d^{d} k\left(\partial_{\mu} D_{L}\right)^{2}\left(1-D_{L}\right)^{-4} \sum_{\nu} \int d^{d} l \hat{G}_{\mu}^{2}\left(1-D_{L}\right)^{-2}\right]^{1 / 2} \\
& \quad \leqq O(\varepsilon) W_{L}^{1 / 2} \leqq O\left(\varepsilon^{3 / 2}\right)
\end{aligned}
$$

Finally we consider the term

$$
\left|\sum_{\mu=1}^{d}(2 \pi)^{-d} \int d^{d} k \hat{\tau}^{3} \hat{G}^{-1} \partial_{\mu}^{2} D_{L} e^{i k \cdot a}\right| \leqq \text { const } \int d^{d} k \sum_{\mu=1}^{d}\left|\partial_{\mu}^{2} D_{L}\right|\left(1-D_{L}\right)^{-3} .
$$

The integral on the right side of (5.24) is bounded above by $O(\varepsilon)$, by condition (5.13).

As a result of the above estimates, we have

$$
W_{a} \leqq c \varepsilon,
$$

where $c$ is independent of $\varepsilon$ and $K^{\prime}$. Choosing $K^{\prime}=c / 3$ gives the desired result.

(e) The bound on $H$ : The argument of Sect.4.3.3(e) can be used here. The condition (5.14) is used.

\subsection{Proof of Lemma 5.1}

In this section we prove Lemma 5.1, which together with Sect. 5.1 completes the proof of Theorem 1.2.

5.2.1. Verification of Conditions (5.6-8). The gaussian critical value $p_{L}$ is given by

$$
p_{L}=\left[\sum_{x} L^{-d} g(x / L)\right]^{-1}
$$


The sum over $x$ in this expression is a Riemann sum approximation to $\|g\|_{1}=1$, and hence

By definition,

$$
\lim _{L \rightarrow \infty} p_{L}=1
$$

$$
S=p_{L} L^{2} \sum_{x} L^{-d}(x / L)^{2} g(x / L) \sim L^{2}\left\|x^{2} g\right\|_{1},
$$

and hence $S=O\left(L^{2}\right)$. Since $g \in L_{\infty}$, it follows from (5.2) that $\sup _{x} p_{0 x}^{(L)}=O\left(L^{-d}\right)$. Since $g \in L_{2}$,

$$
\sum_{x}\left(p_{0 x}^{(L)}\right)^{2}=p_{L}^{2} L^{-d} \sum_{x} L^{-d} g(x / L)^{2} \sim L^{-d}\|g\|_{2}^{2}=O\left(L^{-d}\right) .
$$

This gives the condition (5.6), if $L$ is taken sufficiently large.

Similarly, it follows from the fact that $x^{2} g \in L_{\infty}$ that

$$
\sup _{x} p_{0 x}^{(L)}|x|^{2}=O\left(L^{-d+2}\right),
$$

which yields (5.7). Finally, the fact that $x^{2} g \in L_{2}$ implies

$$
\sum_{x}\left(p_{0 x}^{(L)}\right)^{2}|x|^{4}=O\left(L^{-d+4}\right),
$$

yielding (5.8). The big $O$ notation is used to represent an upper bound involving constants which are independent of $L$, but may depend on $d$ and $g$.

5.2.2. Basic Properties of the Gaussian Propagator and (5.9). In this section we show that the condition (5.9) is satisfied, and obtain some further results which will be used to estimate $T_{L}$, etc., in the following sections.

Recall the definitions:

$$
D_{L}(k)=p_{L} \sum_{x} L^{-d} g(x / L) e^{i k \cdot x}
$$

and

$$
\hat{C}_{L}(k)=\left[1-D_{L}(k)\right]^{-1} .
$$

For a function $h$ defined on $\mathbb{R}^{d}$, we define a transformation

$$
\tilde{h}_{L}(k)=p_{L} \sum_{x} L^{-d} h(x / L) e^{i k \cdot x} .
$$

In this notation, $D_{L}(k)=\tilde{g}_{L}(k)$. We also use the notation $|I|$ to represent the cardinality of a set $I \subset\{1, \ldots, d\}$, and write $\partial^{I}=\prod_{v \in I} \frac{\partial}{\partial x_{v}}$. The expressions $\partial^{I} h$ and $\left\|\partial^{I} h\right\|_{1}$ are to be interpreted in terms of distributions.

Lemma 5.4. Suppose that $h$ vanishes at infinity, that $\frac{\partial^{d} h}{\partial x_{1} \partial x_{2} \cdots \partial x_{d}}$ is piecewise continuous, and that $\tilde{h}_{L}$ exists for all $L$. Then for $L$ sufficiently large,

$$
\left|\tilde{h}_{L}(k)\right| \leqq 2\|h\|_{1} \text {. }
$$


Also, for any $I \subset\{1, \ldots, d\}$ and for any $k$,

$$
\left|\tilde{h}_{L}(k)\right| \leqq 2\left\|\partial^{I} h\right\|_{1}\left|\prod_{v \in I} 2 L \sin \left(k_{v} / 2\right)\right|^{-1} .
$$

Proof. The bound (5.27) follows from (5.25) and the fact that $\sum_{x} L^{-d}|h(x / L)|$ $\rightarrow\|h\|_{1}$ as $L \rightarrow \infty$. The bound (5.28) is proved using summation by parts, as follows. To simplify the notation, we assume that $1 \in I$. Then

$$
\begin{aligned}
\tilde{h}_{L}(k) & =p_{L} \sum_{x} L^{-d} h(x / L) e^{-i k_{1} / 2}\left[e^{i k_{1}\left(x_{1}+1\right)}-e^{i k_{1} x_{1}}\right]\left[2 i \sin \left(k_{1} / 2\right)\right]^{-1} \exp \left[\sum_{v=2}^{d} i k_{v} x_{v}\right] \\
& =-p_{L} e^{-i k_{1} / 2}\left[2 i \sin \left(k_{1} / 2\right)\right]^{-1} \sum_{x} L^{-d}\left[h(x / L)-h\left(x / L-\hat{e}_{1} / L\right)\right] e^{i k \cdot x},
\end{aligned}
$$

where $\hat{e}_{1}=(1,0, \ldots 0)$. To avoid nonilluminating complications, we consider henceforth only the case of continuously differentiable $h$; the general case can be treated similarly. By the mean value theorem (5.29) is equal to

$$
-p_{L} e^{-i k_{1} / 2}\left[2 i \sin \left(k_{1} / 2\right)\right]^{-1} \sum_{x} L^{-d} \frac{1}{L} \partial_{1} h\left(x^{*} / L\right) e^{i k \cdot x},
$$

where $x^{*}$ is a point on the line joining $x / L$ to $\left(x-\hat{e}_{1}\right) / L$. Iteration of this procedure gives

$$
\tilde{h}_{L}(k)=(-1)^{|I|} p_{L} \prod_{v \in I}\left[e^{-i k_{v} / 2} / 2 L i \sin \left(k_{v} / 2\right)\right] \sum_{x} L^{-d} \partial^{I} h\left(x^{* *} / L\right) e^{i k \cdot x},
$$

where $\left\|\left(x^{* *}-x\right) / L\right\|_{\infty} \leqq L^{-1}$. Therefore

$$
\left|\tilde{h_{L}}(k)\right| \leqq p_{L} \prod_{v \in I}\left|2 L \sin \left(k_{v} / 2\right)\right|^{-1} \sum_{x} L^{-d}\left|\partial^{I} h\left(x^{* *} / L\right)\right| \sim \prod_{v \in I}\left|2 L \sin \left(k_{v} / 2\right)\right|^{-1}\left\|\partial^{I} h\right\|_{1} .
$$

This gives the desired bound.

The following lemma is proved using Lemma 5.4. Fix $M>0$ such that the integral of $g$ over the region $\|x\|_{1} \leqq M$ is strictly positive. We write $\delta=3 \pi\left\|\partial_{1} g\right\|_{1} / 2 L$ and $\delta_{1}=\pi / L M$, and use $c$ to denote a constant which is independent of $L$ and whose value may change from one occurrence to another. We also fix a small $\varepsilon>0$ (e.g., $\varepsilon=1 / 5$ ). It seems likely that the bounds in which $\varepsilon$ appears, in the remainder of this section, can be strengthened to the corresponding $\varepsilon=0$ bounds; however the bounds we obtain are sufficient for our needs.

\section{Lemma 5.5.}

$$
\lim _{L \rightarrow \infty} D_{L}(k)=0, \quad \text { for all } k \neq 0 .
$$

For L sufficiently large, the following inequalities hold:

$$
\begin{gathered}
\left|D_{L}(k)\right| \leqq \frac{2}{3}, \quad \text { for }\|k\|_{\infty} \geqq \delta, \\
1-D_{L}(k) \geqq c k^{2} L^{2}, \quad \text { for }\|k\|_{\infty} \leqq \delta_{1}, \\
1-D_{L}(k) \geqq c k^{2} L^{2-\varepsilon}, \text { for } \delta_{1} \leqq\|k\|_{\infty} \leqq \delta\left(\text { if } \delta_{1}<\delta\right),
\end{gathered}
$$


$\left|\partial_{\mu}^{s} D_{L}(k)\right| \leqq 2 L^{s}\left\|\partial^{I}\left(x_{\mu}^{s} g\right)\right\|_{1} \prod_{v \in I}\left|2 L \sin \left(k_{v} / 2\right)\right|^{-1}, \quad$ for $s=0,1,2$ and all $k, I$,

$$
\left|\partial_{\mu} D_{L}(k)\right| \leqq 2 L^{2}\left|k_{\mu}\right|\left\|x_{\mu}^{2} g\right\|_{1}, \quad \text { for all } k
$$

Proof. Equation (5.30) follows from (5.28) with $h=g$ and $I=\{\mu\}, \mu=1, \ldots, d$. To prove (5.31), suppose that $\left|k_{v}\right| \geqq \delta$, and let $I=\{v\}$. Then by (5.28) and the definition of $\delta$,

$$
\left|D_{L}(k)\right| \leqq \pi L^{-1}\left|k_{v}\right|^{-1}\left\|\partial_{v} g\right\|_{1} \leqq 2 / 3
$$

To prove (5.32) we suppose $\|k\|_{\infty} \leqq \delta_{1}$, and use symmetry to write

$$
\begin{aligned}
1-D_{L}(k) & =p_{L} \sum_{x} L^{-d} g(x / L)(1-\cos k \cdot x) \geqq p_{L} 2 \pi^{-2} \sum_{x:|k \cdot x| \leqq \pi} L^{-d} g(x / L)(k \cdot x)^{2} \\
& \geqq p_{L} 2 \pi^{-2} \sum_{x:\|x\|_{1} \leqq L M} L^{-d} g(x / L)(k \cdot x)^{2} \\
& =p_{L} 2 \pi^{-2} k^{2} L^{2} \sum_{x:\|x\|_{1} \leqq L M} L^{-d} g(x / L)\left(x_{1} / L\right)^{2} \\
& \sim 2 \pi^{-2} k^{2} L^{2} \int_{\|x\|_{1} \leqq M} g(x) x_{1}^{2} d^{d} x>c k^{2} L^{2},
\end{aligned}
$$

for $L$ sufficiently large.

The inequality (5.33) is proved as follows. Fix $k$ such that $\delta_{1} \leqq\|k\|_{\infty} \leqq \delta$, and let $X_{k}=\left\{x: 1-\cos k \cdot x>L^{-\varepsilon}\right\}$. Then

$$
\begin{gathered}
1-D_{L}(k) \geqq p_{L} L^{-\varepsilon} \sum_{x \in X_{k}} L^{-d} g(x / L) \geqq k^{2} d^{-1} \delta^{-2} L^{-\varepsilon} p_{L} \sum_{x \in X_{k}} L^{-d} g(x / L) \\
\sim c k^{2} L^{2-\varepsilon}\left[1-\sum_{x \notin X_{k}} L^{-d} g(x / L)\right] .
\end{gathered}
$$

It suffices to show that the sum on the right side goes to zero as $L$ goes to infinity. The domain of summation consists of those $x$ for which $1-\cos k \cdot x \leqq L^{-\varepsilon}$. Since for any $x$ we can find an integer $n$ (depending on $k \cdot x$ ) such that $1-\cos k \cdot x \geqq 2 \pi^{-2}(k \cdot x-2 n \pi)^{2}$, this domain is contained in the set of all $x$ such that $x / L \in Y_{k}$, where

$$
Y_{k}=\left\{y \in Z^{d}:|\hat{k} \cdot y-2 n \pi|^{2} \leqq \frac{\pi^{2}}{2} L^{-\varepsilon} \text {, for some } n \in \mathbb{Z}\right\} .
$$

Here we have written $\hat{k} \equiv k L$, with $\|\hat{k}\|_{\infty} \in\left[\pi / M, 3 \pi\left\|\partial_{1} g\right\|_{1} / 2\right]$. For $R>0$, let $B_{R}=\left\{y:\|y\|_{\infty} \leqq R\right\}$. Since $g$ decays exponentially, given $\varrho>0$ we can choose an $R$ such that

$$
\sum_{\frac{x}{L} \notin B_{R}} L^{-d} g(x / L)<\varrho .
$$

But then we can choose $L$ sufficiently large that

$$
\sum_{\frac{x}{L} \in Y_{\boldsymbol{k}} \cap \boldsymbol{B}_{R}} L^{-d} g(x / L)<\varrho,
$$


since the sum on the left side converges to zero as $L$ goes to infinity, because $g$ is bounded and the measure of $Y_{k} \cap B_{R}$ goes to zero as $L$ goes to infinity. This proves (5.33).

The inequality (5.34) follows from (5.27) and (5.28). To prove (5.35) we use symmetry to write

$$
\begin{aligned}
\left|\partial_{\mu} D_{L}(k)\right| & =\left|p_{L} \sum_{x} L^{-d} g(x / L) x_{\mu} \sin \left(k_{\mu} x_{\mu}\right) \prod_{v \neq \mu} e^{i k_{v} x_{v}}\right| \\
& \leqq p_{L} \sum_{x} L^{-d} g(x / L)\left|x_{\mu}\right|\left|k_{\mu} x_{\mu}\right| \sim L^{2}\left|k_{\mu}\right|\left\|x_{\mu}^{2} g\right\|_{1} .
\end{aligned}
$$

We now use the bounds given in Lemma 5.5 to estimate $\hat{C}_{L}(k)$. These estimates will in turn be used to estimate $T_{G}$ and $W_{G}$. The condition (5.9) is an immediate consequence of (5.37) below, if we take $L$ sufficiently large.

Lemma 5.6. For

In addition

$$
k \neq 0, \quad \hat{C}_{L}(k) \rightarrow 1 \text { as } L \rightarrow \infty .
$$

$$
\begin{array}{r}
0 \leqq \hat{C}_{L}(k) \leqq\left\{\begin{array}{ll}
3 & \text { if }\|k\|_{\infty} \geqq \delta \\
O\left(L^{-2+\varepsilon}\right)|k|^{-2} & \text { if }\|k\|_{\infty} \leqq \delta
\end{array},\right. \\
\left|\hat{C}_{L}(k)-1\right| \leqq\left\{\begin{array}{ll}
3\left|D_{L}(k)\right| & \text { if }\|k\|_{\infty} \geqq \delta \\
O\left(L^{-2+\varepsilon}\right)|k|^{-2} & \text { if }\|k\|_{\infty} \leqq \delta
\end{array},\right. \\
\left|\partial_{\mu} \hat{C}_{L}(k)\right| \leqq \begin{cases}9\left|\partial_{\mu} D_{L}(k)\right| & \text { if }\|k\|_{\infty} \geqq \delta \\
O\left(L^{-2+2 \varepsilon}\right)\left|k_{\mu}\right||k|^{-4} & \text { if }\|k\|_{\infty} \leqq \delta\end{cases}
\end{array}
$$

Here the big $O$ denotes upper bounds involving constants which are independent of $L$, but may depend on $d$ or $g$. Also $|k|$ denotes the euclidean length of $k$.

Proof. The limit (5.36) follows immediately from (5.4) and (5.30). For (5.37), we use (5.31-33). Since $\hat{C}_{L}(k)-1=D_{L}(k) /\left[1-D_{L}(k)\right]$, the first bound in (5.38) follows from (5.31), and the second from (5.32-33) and the fact that $\left|D_{L}(k)\right| \leqq 1$. Similarly (5.39) follows from calculation of the derivative, (5.31-33) and (5.35).

5.2.3. Conditions (5.10), (5.11), (5.14). The following lemma will be used to estimate $T_{G}$ for this model.

Lemma 5.7. For $d>2$,

$$
(2 \pi)^{-d} \int d^{d} k\left|\hat{C}_{L}(k)-1\right| \leqq O\left(L^{-d+\varepsilon}\right)\left[(\ln L)^{d}+\frac{c_{d}}{d-2}\right] .
$$

For $d>2 N, N \geqq 2$,

$$
(2 \pi)^{-d} \int d^{d} k\left|\hat{C}_{L}(k)-1\right|^{N} \leqq O\left(L^{-d+\varepsilon N}\right)\left[1+\frac{c_{d}^{\prime}}{d-2 N}\right] .
$$

The constants $c_{d}$ and $c_{d}^{\prime}$ depend only on $d$ and remain bounded as $d \downarrow 2$ in the first bound and $d \downarrow 2 N$ in the second. 
Proof. We divide the domain of integration into two parts: $\|k\|_{\infty} \leqq \delta$ and $\|k\|_{\infty} \geqq \delta$, and use (5.38) to bound the integrand. This gives

$$
\begin{aligned}
(2 \pi)^{-d} \int d^{d} k\left|\hat{C}_{L}(k)-1\right|^{N} \leqq & 3^{N}(2 \pi)^{-d} \int_{\|k\|_{\infty} \geqq \delta} d^{d} k\left|D_{L}(k)\right|^{N} \\
& +O\left(L^{-(2-\varepsilon) N}\right)(2 \pi)^{-d} \int_{\|k\|_{\infty} \leqq \delta} d^{d} k|k|^{-2 N} \\
\leqq & 3^{N}(2 \pi)^{-d} \int_{\|k\|_{\infty} \geqq \delta} d^{d} k\left|D_{L}(k)\right|^{N} \\
& +O\left(L^{-(2-\varepsilon) N}\right) \Omega_{d}(2 \pi)^{-d} \frac{(\sqrt{d} \delta)^{d-2 N}}{d-2 N}
\end{aligned}
$$

where $\Omega_{d}$ is the volume of the unit sphere in $\mathbb{R}^{d}$. The first term on the right side is estimated using (5.34), as follows.

The region $\|k\|_{\infty} \geqq \delta$ is the disjoint union, over $I \subset\{1,2, \ldots d\}, I \neq \phi$, of

$$
R_{I} \equiv\left\{k \in \mathbb{R}^{d}|\delta<| k_{v} \mid<\pi \text { for } v \in I,\left|k_{\mu}\right| \leqq \delta \text { for } \mu \notin I\right\} .
$$

By (5.34),

$$
\begin{aligned}
(2 \pi)^{-d} \int_{R_{I}} d^{d} k\left|D_{L}(k)\right|^{N} & \leqq 2^{N}\left\|\partial^{I} g\right\|_{1}^{N}(2 \pi)^{-d} \int_{R_{I}} d^{d} k \prod_{v \in I}\left[\pi\left(2 L\left|k_{v}\right|\right)^{-1}\right]^{N} \\
& \leqq c^{N} L^{-N|I|} \delta^{d-|I|} \begin{cases}|\ln \delta|^{|I|} & \text { if } N=1 \\
\delta^{|I|(1-N)} & \text { if } N>1\end{cases}
\end{aligned}
$$

This gives the bounds in the statement of the lemma.

Corollary 5.8. For $d>2$,

For $d>6$,

$$
\left|C_{L}(0,0)-1\right| \leqq O\left(L^{-d+\varepsilon}\right)\left[(\ln L)^{d}+\frac{c_{d}}{d-2}\right] .
$$

$$
T_{L} \leqq O\left(L^{-d+3 \varepsilon}\right)\left(1+\frac{c_{d}^{\prime}}{d-6}\right) .
$$

Proof. The first inequality is an immediate consequence of the first statement in Lemma 5.7. The second follows from the second statement in the lemma and the fact that by definition

$$
\begin{aligned}
T_{L} & =\sum_{x, y} C_{L}(0, x) C_{L}(x, y) C_{L}(y, 0)-C_{L}(0,0)^{3} \\
& =(2 \pi)^{-d} \int d^{d} k\left(\hat{C}_{L}(k)+2\right)\left(\hat{C}_{L}(k)-1\right)^{2}-\left(C_{L}(0,0)+2\right)\left(C_{L}(0,0)-1\right)^{2} .
\end{aligned}
$$

In view of (5.26), Corollary 5.8 ensures that the conditions (5.10) and (5.11) are satisfied for sufficiently large $L$. The condition (5.14) follows immediately from the following lemma. 
Lemma 5.9. For $d>6$,

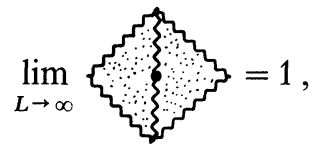

where the lines in the diagram denote the gaussian propagator $\hat{C}_{L}(k)$.

Proof. Fix $d>6$. By (5.36) and (5.37), $\left|\hat{C}_{L}(k)\right|$ is bounded above by $|k|^{-2}$ for $k$ near zero, and $\hat{C}_{L}(k)$ approaches one pointwise as $L$ goes to infinity. Since the replacement of $\hat{C}_{L}(k)$ by $|k|^{-2}$ in the diagram in (5.42) yields a finite result for $d>6$, the conclusion follows by the dominated convergence theorem.

A similar argument can be employed to prove that as $L \rightarrow \infty, T_{L}$ converges to zero and $C_{L}(0,0)$ converges to one. However this is not sufficient for the conditions (5.10-11), and thus we presented the more detailed bounds of Corollary 5.8 .

5.2.4. Bounds on Quantities with Derivatives: Conditions (5.12) and (5.13). The condition (5.12) on $W_{L}$ is an immediate consequence of the following lemma.

Lemma 5.10. For $d>6$,

$$
W_{L} \leqq O\left(L^{2+4 \varepsilon-d}\right)\left(1+\frac{c_{d}}{d-6}\right) .
$$

Proof. By definition,

$$
W_{L}=\sum_{\mu=1}^{d}(2 \pi)^{-d} \int d^{d} k\left(\partial_{\mu} \hat{C}_{L}(k)\right)^{2} .
$$

We divide the domain of integration as in Lemma 5.7 and use (5.39) to obtain

$$
W_{L} \leqq 81(2 \pi)^{-d} \int_{\|k\|_{\infty} \geqq \delta} d^{d} k\left|\partial_{\mu} D_{L}(k)\right|^{2}+O\left(L^{-4+4 \varepsilon}\right) \int_{\|k\|_{\infty} \leqq \delta} d^{d} k|k|^{-6} .
$$

The second term on the right side gives the second term on the right side of (5.43). The integral in the first term on the right side can be estimated by writing the integral as a sum over integrals over $R_{I}$, exactly as was done in the proof of Lemma 5.7, and using (5.34) with $s=1$. The only difference here is the extra factor of $L^{2}$ which arises from $s=1$ in (5.34).

Finally we prove the following bound, which gives the condition (5.13).

Lemma 5.11. For $d>6$,

$$
(2 \pi)^{-d} \int d^{d} k\left|\partial_{\mu}^{2} D_{L}\right|\left(1-D_{L}\right)^{-3} \leqq O\left(L^{2+3 \varepsilon-d}\right)\left[(\ln L)^{d}+\frac{c_{d}}{d-6}\right] .
$$


Proof. Using (5.37) and (5.33) gives

$$
\begin{aligned}
(2 \pi)^{-d} \int d^{d} k\left|\partial_{\mu}^{2} D_{L}\right|\left(1-D_{L}\right)^{-3} \leqq & 27(2 \pi)^{-d} \int_{\|k\|_{\infty} \geqq \delta} d^{d} k\left|\partial_{\mu}^{2} D_{L}\right| \\
& +O\left(L^{-6+3 \varepsilon+2}\right) \int_{\|k\|_{\infty} \leqq \delta} d^{d} k|k|^{-6} .
\end{aligned}
$$

The second term on the right side gives the second term on the right side of (5.44). For the integral over $\|k\|_{\infty} \geqq \delta$ we use (5.34) with $s=2$, as in Lemmas 5.7 and 5.10, to obtain the desired result.

Acknowledgements. It is a pleasure to thank Michael Aizenman for many stimulating and valuable conversations concerning the subject of this paper. G.S. also wishes to thank him for making possible a visit to the Courant Institute in May of 1988, during which this work was begun. We thank David Brydges, Chuck Newman, Alan Sokal, and Hal Tasaki for interesting and useful conversations.

\section{References}

1. Aizenman, M.: Geometric analysis of $\varphi^{4}$ fields and Ising models, Parts I and II. Commun. Math. Phys. 86, 1-48 (1982)

2. Aizenman, M., Barsky, D.J.: Sharpness of the phase transition in percolation models. Commun. Math. Phys. 108, 489-526 (1987)

3. Aizenman, M., Fernández, R.: On the critical behaviour of the magnetization in high dimensional Ising models. J. Stat. Phys. 44, 393-454 (1986)

4. Aizenman, M., Graham, R.: On the renormalized coupling constant and the susceptibility in $\lambda \varphi_{d}^{4}$ field theory and the Ising model in four dimensions. Nucl. Phys. B225 [FS9], 261-288 (1983)

5. Aizenman, M., Kesten, H., Newman, C.M.: Uniqueness of the infinite cluster and continuity of connectivity functions for short and long range percolation. Commun. Math. Phys. 111, 505-531 (1987)

6. Aizenman, M., Newman, C.M.: Tree graph inequalities and critical behaviour in percolation models. J. Stat. Phys. 36, 107-143 (1984)

7. Aizenman, M., Simon, B.: Local Ward identities and the decay of correlations in ferromagnets. Commun. Math. Phys. 77, 137-143 (1980)

8. Barsky, D.J., Aizenman, M.: Percolation critical exponents under the triangle condition. Preprint (1988)

9. van den Berg, J., Kesten, H.: Inequalities with applications to percolation and reliability, J. Appl. Prob. 22, 556-569 (1985)

10. Broadbent, S.R., Hammersley, J.M.: Percolation processes. I. Crystals and mazes. Proc. Camb. Phil. Soc. 53, 629-641 (1957)

11. Brydges, D.C., Fröhlich, J., Sokal, A.D.: A new proof of the existence and nontriviality of the continuum $\varphi_{2}^{4}$ and $\varphi_{3}^{4}$ quantum field theories. Commun. Math. Phys. 91, 141-186 (1983)

12. Brydges, D.C., Spencer, T.: Self-avoiding walk in 5 or more dimensions. Commun. Math. Phys. 97, 125-148 (1985)

13. Chayes, J.T., Chayes, L.: On the upper critical dimension of Bernoulli percolation. Commun. Math. Phys. 113, 27-48 (1987)

14. Essam, J.W.: Percolation Theory. Rep. Prog. Phys. 43, 833-912 (1980)

15. Fröhlich, J.: On the triviality of $\varphi_{d}^{4}$ theories and the approach to the critical point in $d_{(\geqq)} 4$ dimensions. Nucl. Phys. B200 [FS4], 281-296 (1982)

16. Fröhlich, J., Simon, B., Spencer, T.: Infrared bounds, phase transitions, and continuous symmetry breaking. Commun. Math. Phys. 50, 79-95 (1976) 
17. Grimmett, G.: Percolation, Berlin Heidelberg New York: Springer 1989

18. Hammersley, J.M.: Percolation processes. Lower bounds for the critical probability. Ann. Math. Statist. 28, 790-795 (1957)

19. Hammersley, J.M.: Bornes supérieures de la probabilité critique dans un processus de filtration. In: Le Calcul des Probabilités et ses Applications 17-37 CNRS Paris (1959)

20. Hara, T.: Mean field critical behaviour of correlation length for percolation in high dimensions. Preprint (1989)

21. Hara, T., Slade, G.: On the upper critical dimension of lattice trees and lattice animals. Submitted to J. Stat. Phys.

22. Hara, T., Slade, G.: Unpublished

23. Kac, M., Uhlenbeck, G.E., Hemmer, P.C.: On the van der Waals theory of the vapor-liquid equilibrium. I. Discussion of a one-dimensional model. J. Math. Phys. 4, 216-288 (1963)

24. Kesten, H.: Percolation theory and first passage percolation. Ann. Probab. 15, 1231-1271 (1987)

25. Lawler, G.: The infinite self-avoiding walk in high dimensions. To appear in Ann. Probab. (1989)

26. Lebowitz, J.L., Penrose, O.: Rigorous treatment of the van der Waals-Maxwell theory of the liquid-vapor transition. J. Math. Phys. 7, 98-113 (1966)

27. Menshikov, M.V., Molchanov, S.A., Sidorenko, A.F.: Percolation theory and some applications, Itogi Nauki i Tekhniki (Series of Probability Theory, Mathematical Statistics, Theoretical Cybernetics) 24, 53-110 (1986). English translation. J. Soviet Math. 42, 17661810 (1988)

28. Nguyen, B.G.: Gap exponents for percolation processes with triangle condition. J. Stat. Phys. 49, 235-243 (1987)

29. Park, Y.M.: Direct estimates on intersection probabilities of random walks. To appear in J. Stat. Phys.

30. Russo, L.: On the critical percolation probabilities. Z. Wahrsch. Verw. Gebiete. 56, 229-237 (1981)

31. Slade, G.: The diffusion of self-avoiding random walk in high dimensions. Commun. Math. Phys. 110, 661-683 (1987)

32. Slade, G.: The scaling limit of self-avoiding random walk in high dimensions. Ann. Probab. 17, 91-107 (1989)

33. Slade, G.: Convergence of self-avoiding random walk to Brownian motion in high dimensions. J. Phys. A: Math. Gen. 21, L417-L420 (1988)

34. Slade, G.: The lace expansion and the upper critical dimension for percolation, Lectures notes from the A.M.S. Summer Seminar, Blacksburg, June 1989

35. Sokal, A.D.: A rigorous inequality for the specific heat of an Ising or $\varphi^{4}$ ferromagnet. Phys. Lett. 71 A, 451-453 (1979)

36. Sokal, A.D., Thomas, L.E.: Exponential convergence to equilibrium for a class of random walk models. J. Stat. Phys. 54, 797-828 (1989)

37. Stauffer, D.: Introduction to percolation theory. Taylor and Francis, London Philadelphia (1985)

38. Tasaki, H.: Hyperscaling inequalities for percolation. Commun. Math. Phys. 113, 49-65 (1987)

39. Tasaki, H.: Private communication

40. Yang, W., Klein, D.: A note on the critical dimension for weakly self avoiding walks. Prob. Th. Rel. Fields 79, 99-114 (1988)

41. Ziff, R.M., Stell, G.: Critical behaviour in three-dimensional percolation: Is the percolation threshold a Lifshitz point? Preprint (1988)

Communicated by M. Aizenman

Received February 8, 1989; in revised form July 6, 1989 
